Aus der Abteilung Mund-, Kiefer- und Gesichtschirurgie

(Prof. Dr. med. Dr. med. dent. H. Schliephake)

im Zentrum Zahn-, Mund- und Kieferheilkunde

der Medizinischen Fakultät der Universität Göttingen

\title{
Manometrische Untersuchungen der oralen Phase des Schluckvorganges
}

\author{
INAUGURAL - DISSERTATION \\ zur Erlangung des Doktorgrades \\ für Zahnheilkunde \\ der Medizinischen Fakultät der \\ Georg-August-Universität zu Göttingen
}

vorgelegt von

Petra Santander Martinez

aus

Mechernich

Göttingen 2012 
Dekan:

I. Berichterstatter: $\quad$ Prof. Dr. med. Dr. med. dent. W. Engelke

II. Berichterstatter/in: $\quad$ Prof. Dr. med. M. Canis

III. Berichterstatter/in: Prof. Dr. med. M. Oppermann 


\section{Inhaltsverzeichnis}

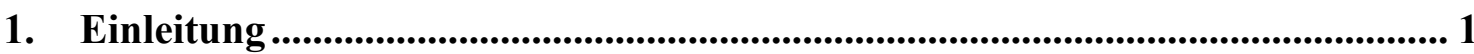

1.1. Allgemeine Anatomie des orofazialen Systems ............................................ 1

1.1.1. Das biofunktionelle Modell ................................................................... 5

1.2. Physiologie und Pathologie des Schluckaktes.................................................. 10

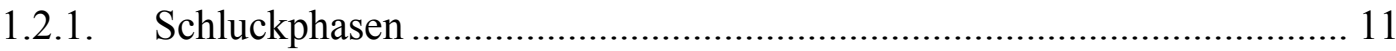

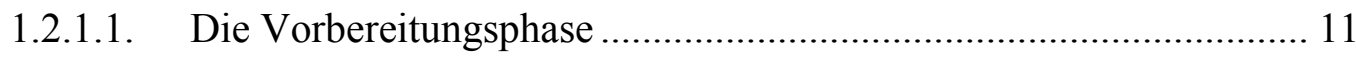

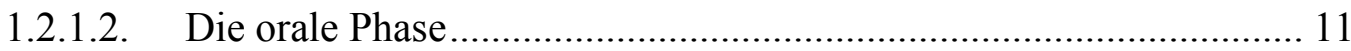

1.2.1.3. Die pharyngeale Phase ................................................................ 12

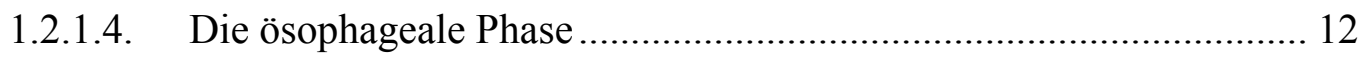

1.2.2. Entwicklung der oralen Saugschluckfunktion ........................................ 13

1.2.3. Habituelle Schluckstörungen .............................................................. 14

1.2.4. Organische Schluckstörungen ...................................................... 15

1.2.4.1. Entwicklungsbedingte neurogene Schluckstörungen........................ 16

1.2.4.2. Erworbene neurogene Schluckstörungen ....................................... 16

1.2.4.3. Angeborene strukturell bedingte Schluckstörungen ....................... 17

1.2.4.4. Erworbene strukturell bedingte Schluckstörungen ......................... 17

1.3. Diagnostik und Beurteilung des Schluckaktes .............................................. 19

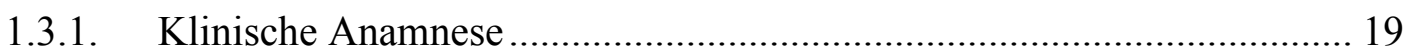

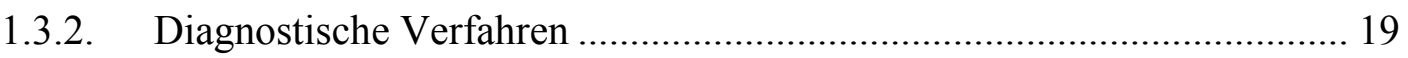




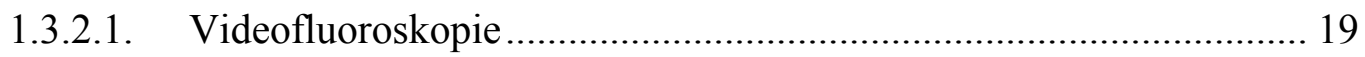

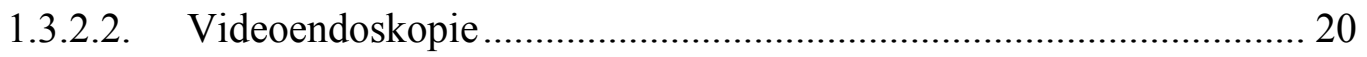

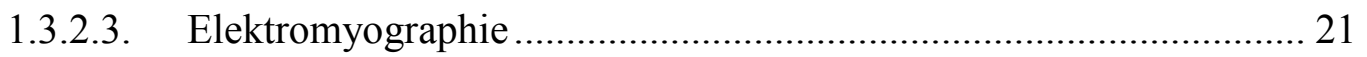

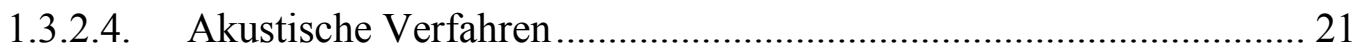

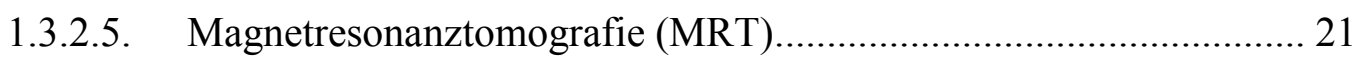

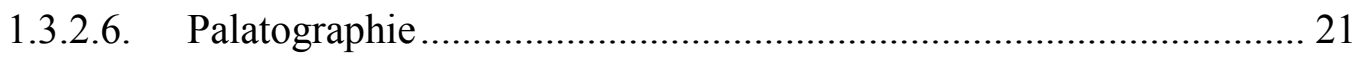

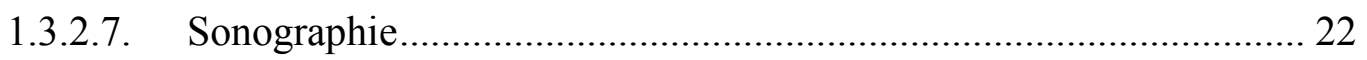

1.3.2.8. Elektromagnetische Artikulographie (EMA) .............................. 22

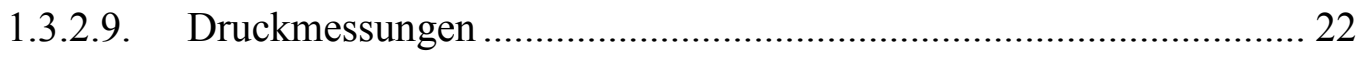

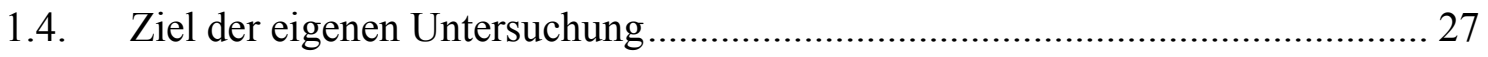

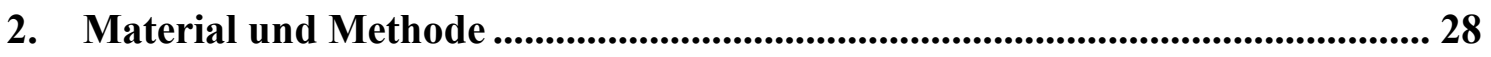

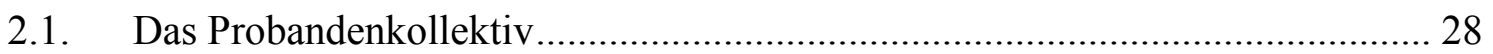

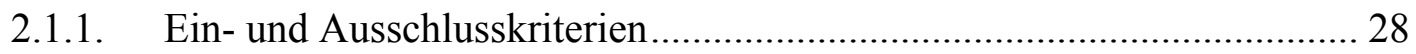

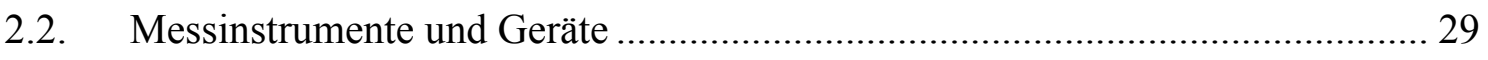

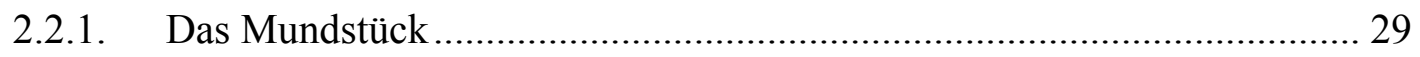

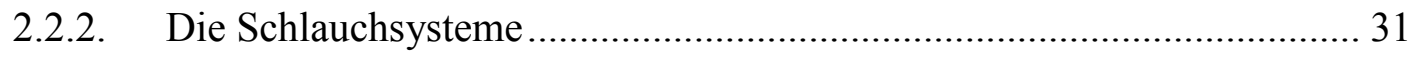

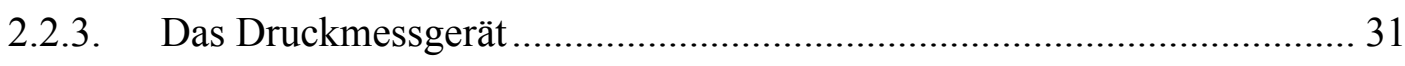

2.2.4. Die verwendeten Flüssigkeiten....................................................... 33

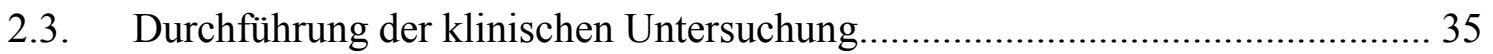

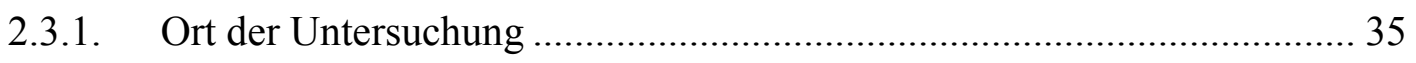

2.3.2. Aufklärung der Probanden............................................................. 35

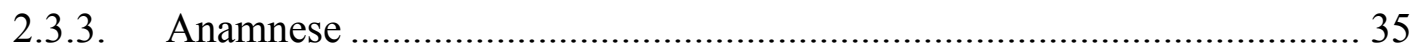


2.3.4. Vorbereitungen der Probanden und Eichung der Geräte. 35

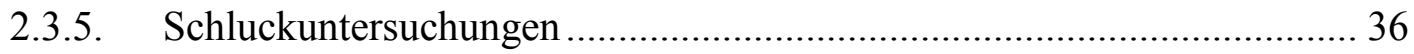

2.3.5.1. Aktive Bolus-Einnahme (ABE) ................................................... 36

2.3.5.2. Passive Wasser-Bolus-Gabe (PWG) .............................................. 37

2.3.5.3. Passive Gel-Bolus-Gabe (PGG) _.................................................. 37

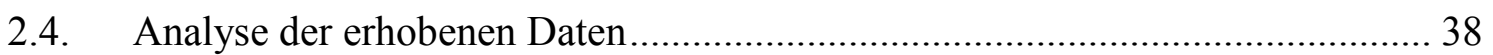

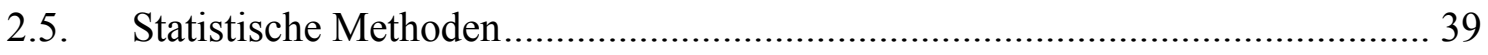

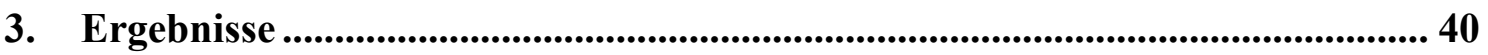

3.1. Allgemeine Beschreibung der Druckverläufe beim Schluckvorgang. ............... 40

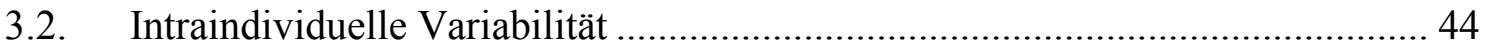

3.3. Qualitative Auswertung in Abhängigkeit von der Schluckmodalität................. 45

3.3.1. Aktive Bolus-Einnahme (ABE) …................................................... 45

3.3.2. Passive Wasser-Bolus-Gabe (PWG) ...................................................... 45

3.3.3. Passive Gel-Bolus-Gabe (PGG) .......................................................... 46

3.4. Quantitative Auswertung in Abhängigkeit von den Modalitäten....................... 48

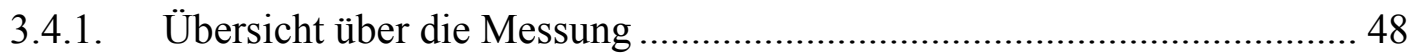

3.4.2. Aktive Bolus-Einnahme (ABE) ......................................................... 50

3.4.3. Passive Wasser-Bolus-Gabe (PWG) ..................................................... 51

3.4.4. Passive Gel-Bolus-Gabe (PGG) ….......................................................... 51

3.5. Spezifische Korrelationen von quantitativen und qualitativen Merkmalen ....... 53

3.5.1. Aktive Bolus-Einnahme (ABE) ......................................................... 53

3.5.2. Passive Wasser-Bolus-Gabe (PWG) ........................................................ 56 


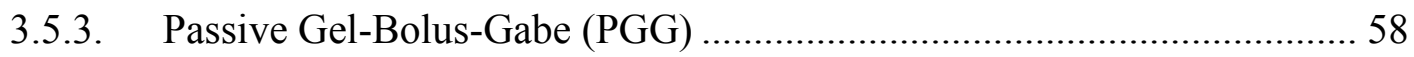

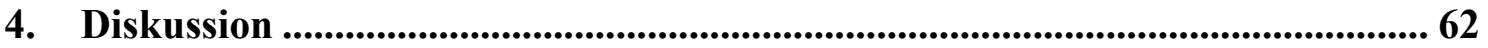

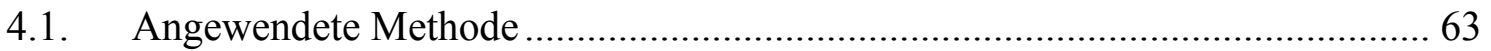

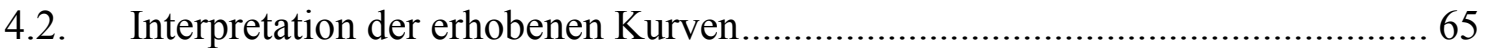

4.2.1. Die aktive Bolus-Einnahme - manometrische Darstellung der

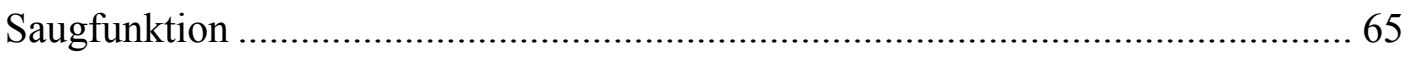

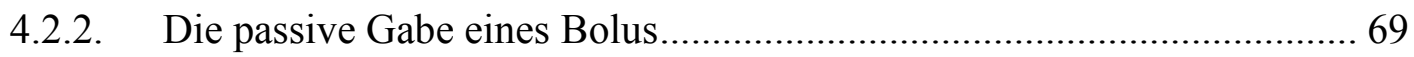

4.3. Darstellung des Schluckvorganges nach dem biofunktionellen Modell ............ 77

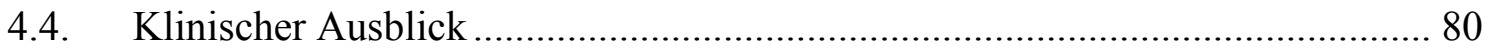

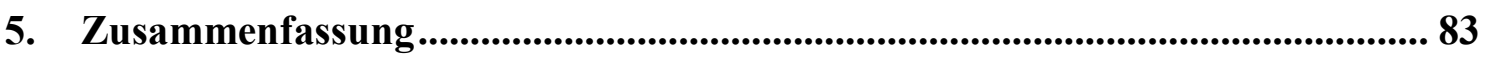

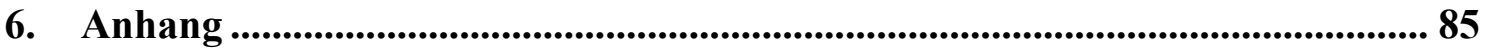

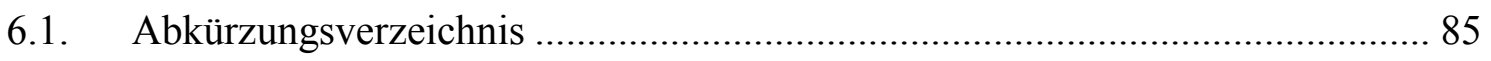

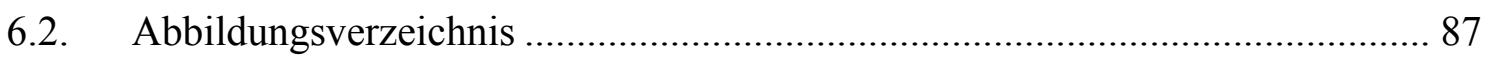

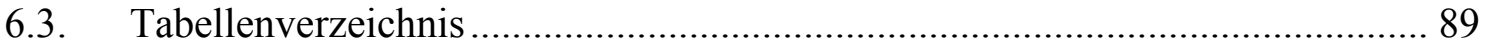

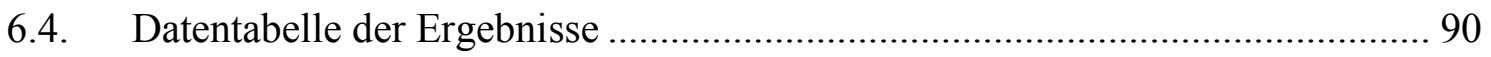

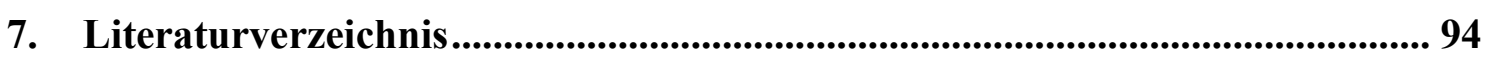




\section{Einleitung}

Das Schlucken ist ein hochkomplexer Mechanismus, der die Nahrungsaufnahme sicherstellt. Dieser Prozess erfordert die koordinierte Bewegung der verschiedenen Strukturen, die den Transport des Bolus in den Magen ermöglichen und den Luftweg schützen. Um den Schluckakt besser zu erfassen, sind Grundkenntnisse der Anatomie und der Physiologie sowie Kenntnisse über verschiedene Untersuchungsmethoden notwendig.

\subsection{Allgemeine Anatomie des orofazialen Systems}

Das orofaziale System umfasst zwei lebenswichtige biologische Aktivitäten - die Nahrungsaufnahme und die Atmung. Soziale Funktionen dieses Systems beziehen sich auf die Kommunikation mit Lautbildung und Mimik sowie auf sensorische Funktionen (Friedrich und Bigenzahn 1995).

Das orofaziale System besteht aus dem nasalen und pharyngealen Luftweg sowie aus der Mundhöhle mit den angrenzenden Strukturen. Zwischen diesen Komponenten gibt es eine enge Beziehung. Komplexe Interaktionen treten im Oropharynx auf, wo die Atmung und der Schluckakt von den Muskeln unterstützt werden (Matsuo und Palmer 2009).

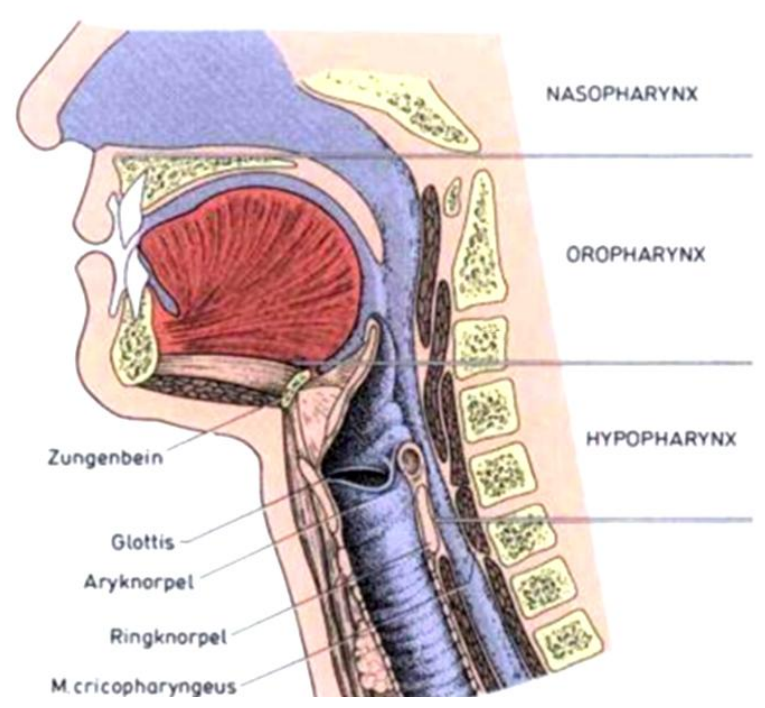

Abbildung 1 Schematische Darstellung des orofazialen Systems (Bartolome und Schröter-Morasch 2010, Seite 2) 
Die Mundhöhle (Cavitas Oris) wird von den Zahnbögen in zwei Räume eingeteilt. Die eigentliche Mundhöhle liegt innerhalb des Zahnbogens. Außerhalb des Zahnbogens liegt der Vorhof, der von Lippen und Gingiva begrenzt ist (Lippert 2006).

Die Kaumuskulatur hängt den Mandibulakörper auf und bestimmt während der Aktivität dessen Position in dreidimensionaler Ebene. Die Kaumuskulatur besteht aus dem M. masseter, dem M. temporalis und den Mm. pterigoidaei. Zusammen mit der mimischen Muskulatur sorgen die Kaumuskeln für die Zerkleinerung des Bolus während des Kauaktes. Dieser beginnt mit dem Abbeißen und endet mit dem Aufbereiten und der Formung eines breiartigen und somit schluckbereiten Bolus (Rohen und Lütjen-Drecoll 2006).

In der Mundhöhle befindet sich die Zunge - ein Muskelkörper mit außergewöhnlich vielfältigen Bewegungsmöglichkeiten. Auch eine aktive Verlängerung der Zunge ist möglich. Dies ist eine alleinige Eigenschaft quergestreifter Muskeln (Felton et al. 2007). Bei der Zunge wird zwischen einer äußeren und inneren Muskulatur unterschieden. Die äußere Zungenmuskulatur wird von Muskelfasern gebildet, die die Zunge in alle drei Raumrichtungen ziehen und für die Zungenbeweglichkeit zuständig sind. Dazu gehören der M. genioglossus, der von der Spina mentalis hauptsächlich zur Aponeurosis linguae läuft und Nebenansätze am Os hyoideum und an der Epiglottis hat; der M. hyoglossus, der seinen Ursprung am Os hyoidei (Corpus und Cornu majus) hat und an der Aponeurosis linguae ansetzt und der M. styloglossus, der vom Processus styloideus zum Seitenrand der Zunge verläuft (Lippert 2006).

Die Binnenmuskulatur der Zunge entspringt aus dem Bindegewebe und hat ihren Ansatz am Zungenkörper. Zur Binnenmuskulatur gehören der M. longitudinalis superior, der unter der Schleimhaut verläuft, und tiefer gelegen der M. longitudinalis inferior. Beide verlaufen der Länge nach in der Zunge von der Zungenspitze bis zum Zungengrund. Sie besitzen die Fähigkeit, die Zunge zu verkürzen und zu verbreitern, sowie die Zungenspitze zu bewegen. Der M. transversus lingualis verläuft quer zu den Mm. longitudinalis. Seine Aufgabe besteht in der Verlängerung und Verschmälerung der Zunge. Der M. verticalis linguae verläuft senkrecht und flacht die Zunge ab beziehungsweise verlängert sie (Dauber und Feneis 2005).

Aufgrund ihres Aufbaus besitzt die Zunge große Bewegungsmöglichkeiten und somit eine vielfältige Funktionalität. Dies ist zum Beispiel bei der Lautbildung, der Sprache 
und bei der oralen Phase des Schluckaktes zu beobachten. Eine Fehlbildung oder Fehlhaltung der Zunge kann orale Funktionen behindern (Lippert 2006).

Kaudal wird die Mundhöhle vom Mundboden begrenzt. Hier verlaufen drei Muskeln: der M. mylohyoideus, der M. geniohyoideus und der M. digastricus. Der $M$. mylohyoideus spannt sich quer auf, er hat seinen Ursprung an der Linea mylohyoidea der Mandibula und verläuft zum Zungenbein. Somit gibt er dem Mundboden eine stabile Basis. Der M. geniohyoideus entspringt von der Spina mentalis und setzt am Zungenbein an. Zu seinen Funktionen gehören die Verstärkung und Verspannung des Mundbodens in Längsrichtung. Diese Funktion weist auch der Venter anterior des M. digastricus auf. Der vordere Anteil dieses zweibauchigen Muskels hat seinen Ursprung am Unterrand des Unterkiefers und verläuft kaudal- und lateralwärts bis zu einer Zwischensehne, die auch den Ansatz für den posterioren Anteil des Muskels bildet, dessen Ursprung sich am Os temporale befindet (Lippert 2006).

Nach kranial wird die Mundhöhle durch den Gaumen begrenzt, der in anterior posteriorer Richtung zu zwei Dritteln aus knöchernem harten Gaumen besteht, und dessen dorsales Drittel durch den weichen Gaumen, beziehungsweise durch das Velum palatini gebildet wird. Seitlich des Velums befinden sich die zwei Gaumenbögen, von anterior nach posterior gesehen der Arcus palatoglossus und der Arcus palatopharyngeus. Mittig des Gaumensegels befindet sich eine zäpfchenartige Struktur, die Uvula, die im entspannten Zustand auf der Zungenwurzel ruht. Das Velum spannt sich segelartig zwischen dem harten Gaumen und dem Nasopharynx auf. Im angespannten Zustand trennt das Velum den Nasopharynx vom Oropharynx (Morris 1988).

Fünf Muskeln bilden das Velum palatini. Der $M$. tensor veli palatini hat seinen Ursprung im Processus pterygoideus und der Lamina membranacea der Tuba auditiva, er zieht nach kaudal zum Hamulus pterygoideus und hat seinen Ansatz in der Aponeurosis palatina. Seine Aufgabe ist die Spannung des Gaumensegels und die Öffnung des Tubeneingangs zum Druckausgleich (Misurva 1976; Lippert 2006).

Der M. levator veli palatini verläuft schräg von der Facies inferior der Pars petrosa des Ossis temporalis und des Cartilago tubae auditivae nach vorne, unten und medianwärts. Oberhalb des Hamulus pterygoideus strahlt er in die Aponeurosis palatina in den weichen Gaumen ein. Er hebt und spannt das Gaumensegel. Zusammen mit dem M. 
constrictor pharyngis superior schließt der $M$. levator veli palatini den Nasenrachenraum beim Schlucken. Der M. uvulae hat seinen Ursprung in der Aponeurosis palatina und seinen Ansatz in der Schleimhaut der Spitze der Uvula palatina, die er auch verkürzen kann.

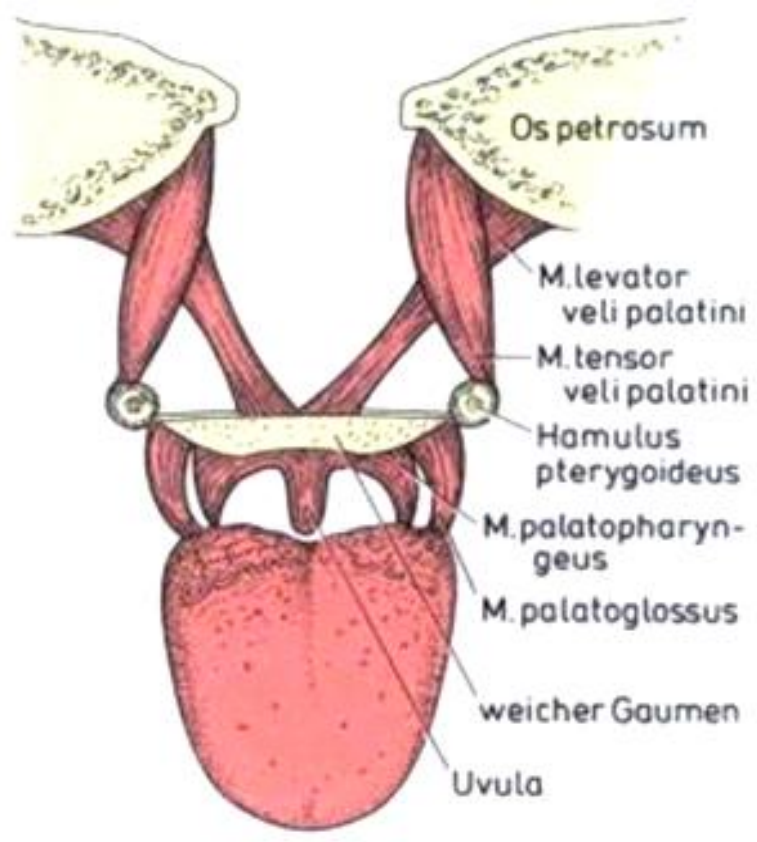

Abbildung 2 Schematische Darstellung der beteiligten Muskeln bei der Anhebung des weichen Gaumens (Bartolome und Schröter-Morasch 2010, Seite 5)

Der Ursprung des M. palatoglossus liegt am Seitenrand der Zunge an einer Abspaltung des M. transversus linguae, setzt an der Aponeurosis palatina an und bildet so die muskuläre Grundlage des vorderen Gaumenbogens. Zu seinen Funktionen zählen das Senken des Gaumensegels und der Verschluss der Schlundenge beim Schlucken. Zwischen beiden Gaumenbögen liegt die Fossa tonsillaris mit der Tonsilla palatina. Der hintere Gaumenbogenmuskel ist der M. palatopharyngeus. Dieser hat seinen Ursprung an der dorsalen Pharynxwand und am Schildknorpel. Sein Ansatz befindet sich an der Aponeurosis palatina. Zu seinen Aufgaben gehören das Senken des Gaumensegels mit Hilfe des M. stylopharyngeus und das Heben des Rachens beim Schlucken (Lippert 2006). 


\subsubsection{Das biofunktionelle Modell}

Dem orofazialen System werden verschiedene Funktionen zugeordnet, daher ist es nicht nur deskriptiv, sondern auch funktionell zu betrachten. Diese Funktionen sind vielfältig und erfordern ein großes Anpassungsvermögen des orofazialen Systems. Nach Engelke et al. (2011) wird das orofaziale System als ein multifunktionelles Hohlorgansystem betrachtet und in einem biofunktionellen Modell systematisch beschrieben. Gegliedert wird das System in zwölf biofunktionelle Einheiten (BFE), die eine integrierte Betrachtung der Physiologie und Anatomie des orofazialen Systems erlaubt (Engelke et al. 2011).

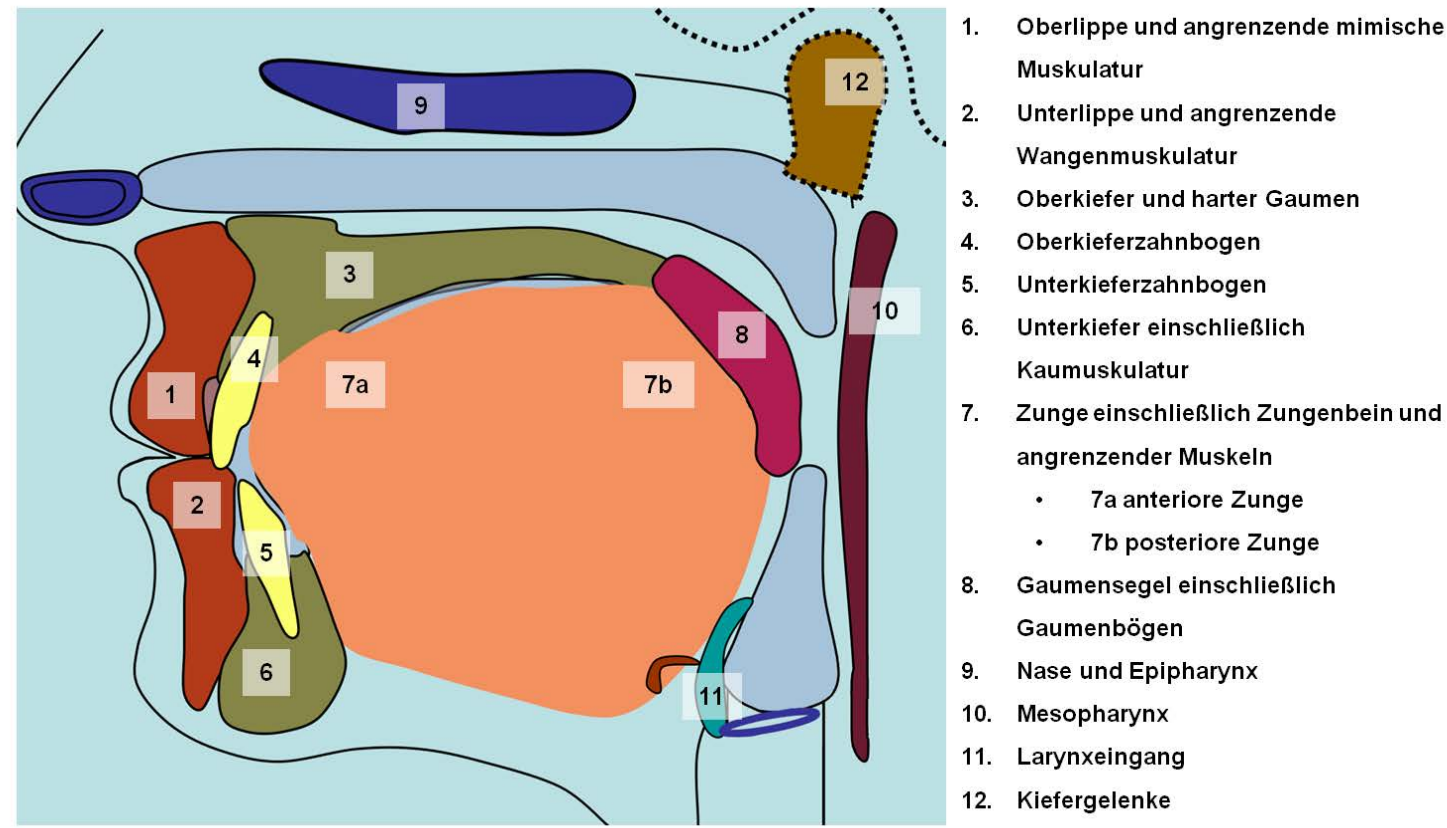

Abbildung 3 Orofaziales System und biofunktionelle Einheiten nach dem biofunktionellen Modell (Modifiziert nach Engelke 2007, Seite 15)

Die biofunktionellen Einheiten besitzen verschiedene Eigenschaften. Bei dem Kontakt zweier Strukturen, wie zum Beispiel zwischen Ober- und Unterlippe, entstehen Verschlüsse. So sind sieben Verschlüsse (V) zu erkennen. Der von diesen Verschlüssen begrenzte Raum kann durch dieses Modell definiert werden. Hierbei handelt es sich um vier biofunktionelle Kompartimente (BFK). Auf diesen Bestandteilen (Einheiten und Verschlüsse, die die Kompartimente begrenzen) beruht das biofunktionelle Modell und erlaubt eine integrierte Betrachtung der Physiologie und Anatomie des orofazialen 
Systems (Engelke et al, 2011). Die Verschlüsse (V1-V7) und die dabei entstehenden Kompartimente (BFK1-BKF4) werden in einem Sagittalschnitt in der Abbildung 4 schematisiert dargestellt.

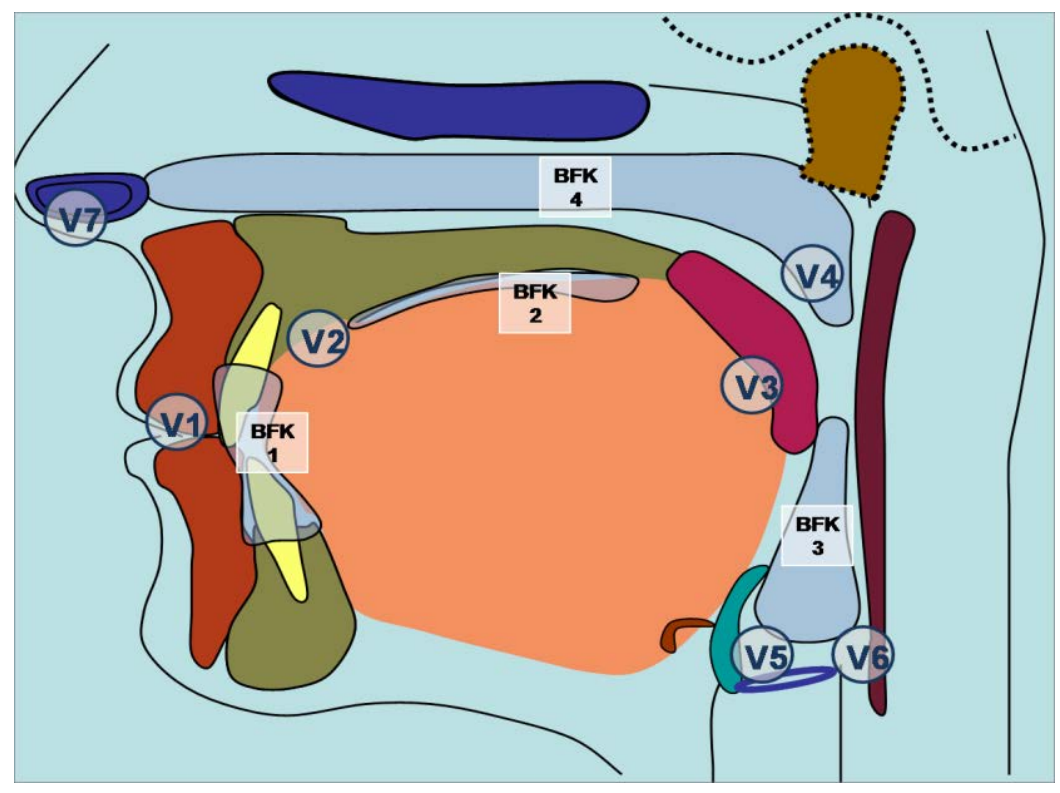

Abbildung 4 Verschlüsse und biofunktionelle Kompartimente nach dem biofunktionellen Modell (Modifiziert nach Engelke 2007, Seite 15)

Die Verschlüsse werden nach den beteiligten Strukturen benannt, so handelt es sich bei V1 um einen Lippenverschluss, bei V2 um den palatolingualen Verschluss, bei V3 um den velolingualen Verschluss und bei V4 um den velopharyngealen Verschluss. V5 wird durch den Verschluss der Glottis gebildet, V6 vom ösophagealer Sphinkter. Ein inkompletter Verschluss ist V7, der Naseneingang.

Die von den Verschlüssen gebildeten biofunktionellen Kompartimente werden nach den jeweiligen anatomischen Grenzen benannt. Hierbei wird das biofunktionelle Kompartiment 1 als interokklusales Kompartiment definiert und entspricht dem Kauschlauch oder dem Raum, der die Zahnreihen umschließt. Nach außen wird es durch die Lippen (V1) und Wangenweichteile begrenzt, nach oral durch den Zungenrand, der den harten Gaumen berührt (V2). Ins Kompartiment 1 münden die Ausführungsgänge der großen Speicheldrüsen. Das biofunktionelle Kompartiment 2 besteht aus dem subpalatinalen Raum und wird vom Gaumengewölbe und der Zungenoberfläche begrenzt (V2). Nach dorsal wird es vom Gaumensegel und dessen Kontakt zum Zungengrund abgegrenzt (V3). Das biofunktionelle Kompartiment 3 liegt im 
Mesopharynx und wird vom velopharyngealen Sphinkter (V4) und den Verschlüssen am Larynx- (V5) und Ösophaguseingang (V6) begrenzt. Bei dem biofunktionellen Kompartiment 4 handelt es sich um ein zur Respiration und Lautbildung dienendes Kompartiment. Dieses wird durch den Kontakt des weichen Gaumens mit der Pharynxwand (V4) und von einem externen manuellen Verschluss des Naseneinganges (V7) gebildet, wie es beim Nasenblähen der Fall ist.

Die Entstehung der obengenannten Verschlüsse und Kompartimente ist von der durchgeführten Bewegung des orofazialen Systems abhängig. Hierbei muss beachtet werden, dass sich die orofazialen Strukturen zu circa 85\% der Zeit in der Ruhelage befinden (Zirkler 2002), was die Bedeutung dieser Position erheblich steigert. Nach dem biofunktionellen Modell wird in eine offene und eine geschlossene Ruhelage unterteilt.

In einer geschlossenen Ruhelage sind Verschlüsse vorhanden. Die Lippen sind entspannt, aber geschlossen, die Kaumuskulatur ist ebenfalls entspannt und der Unterkiefer befindet sich in einer Ruheschwebelage, in der er biofunktionell zentriert ist. Besonders zu betrachten ist die Zungenlage. Der Zungenrücken ist an den Gaumen angelagert. Das Kompartiment 2 wird räumlich minimiert, da das Zungenvolumen den Raum füllt. Das Gaumensegel und die Gaumenbögen liegen entspannt auf dem posterioren Anteil der Zunge (Engelke et al. 2011). In diesem Ruhezustand ist ein negativer Druck in den biofunktionellen Kompartimenten 1 und $2 \mathrm{zu}$ finden, die so zu einem Spaltraum zwischen den benachbarten Strukturen schrumpfen. Bei einer offenen Ruhelage sind keine Verschlüsse zu erkennen. Die Lippen sind geöffnet und es besteht kein Kontakt zwischen Gaumen und Zunge. Der intraorale Druck ist gleich dem extraoralen Druck. Die Lage des Unterkiefers wird hauptsächlich vom Muskeltonus und der Schwerkraft definiert. Die Strukturen sind frei beweglich und nur durch die eigene Anatomie begrenzt. In dieser Lage ist es nicht möglich, eine Definition der Zungen- und Unterkieferposition in der oralen Kavität zu bestimmen (Engelke et al. 2011). Beispiele für eine offene, semi-geschlossene und eine geschlossene Ruhelage sind in der Abbildung 5 gegeben. 


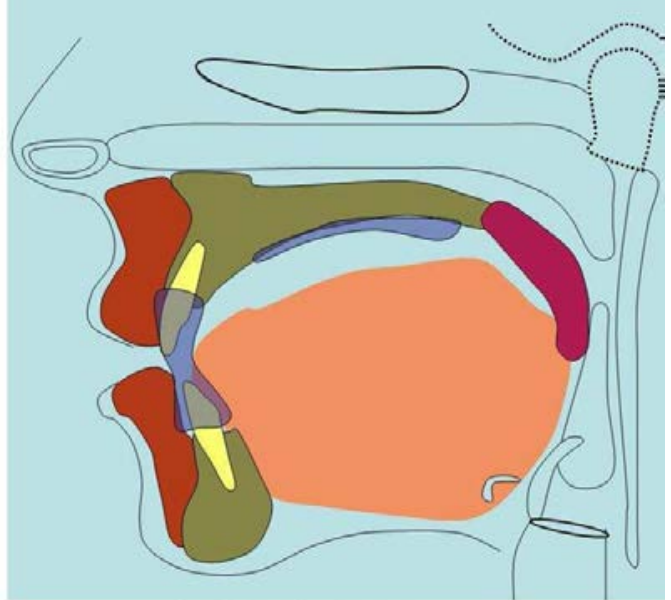

A. Offene Ruhelage

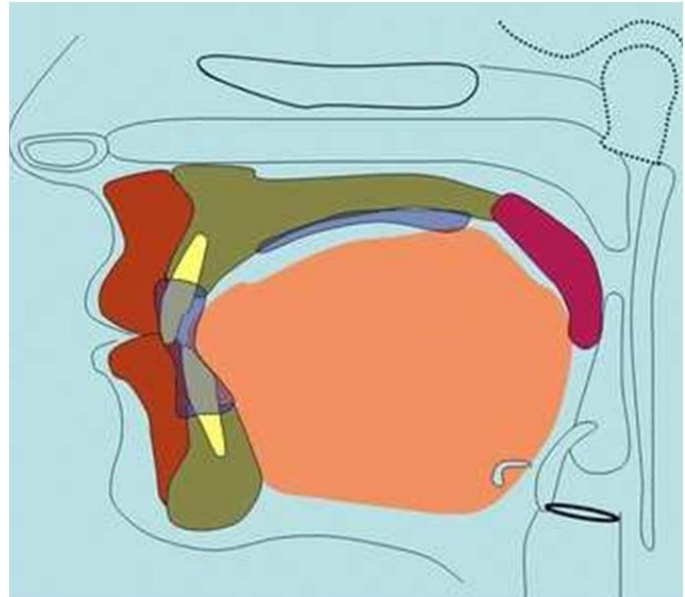

B. Semi-geschlossene Ruhelage

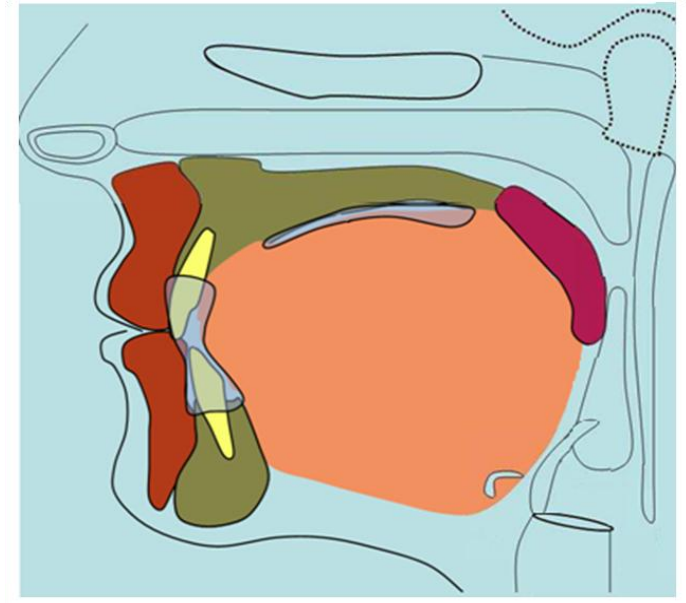

C. Geschlossene Ruhelage

Abbildung 5 Ruhelagen des biofunktionellen Modells (Modifiziert nach Engelke et al. 2011, Seite 167)

Die stabilisierenden Eigenschaften bei einer geschlossenen Ruhelage betreffen besonders die Zunge und ihre Position in Bezug auf die Zahnreihe. Dieser Aspekt ist aus kieferorthopädischer Sicht wichtig, da die Zahnstellung von der Zungenposition beeinflusst wird (Melsen et al. 1987). Des Weiteren hat die Zungenlage bei einer geschlossenen Ruhelage - und daher bei der Entstehung der Verschlüsse V2 und V3 einen schützenden Einfluss auf den pharyngealen Luftweg und verhindert den Kollaps bei schlafbedingten Störungen der Atmung (Engelke et al. 2010). Um eine geschlossene Ruhelage hervorzurufen, ist ein forciertes Schlucken notwendig. Nach Untersuchungen von Engelke et al. (2006) konnte bewiesen werden, dass nach der Ausführung eines kräftigen Schluckens (oder eines Zungenrepositionsmanövers) ein negativer Druck im 
Kompartiment $2 \mathrm{zu}$ finden ist. Auch die Position der orofazialen Strukturen bei einer geschlossen Ruhelage konnte so geprüft werden (Engelke et al. 2006).

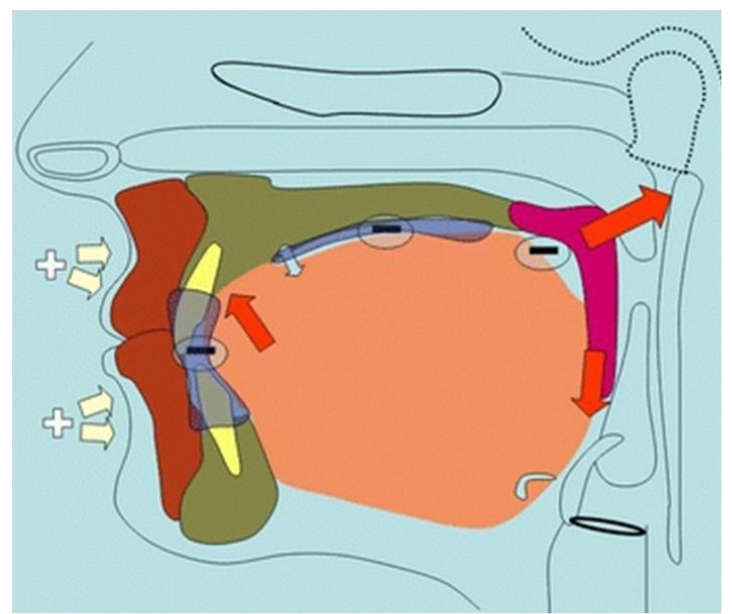

A. Druckverhältnisse während des ZRM

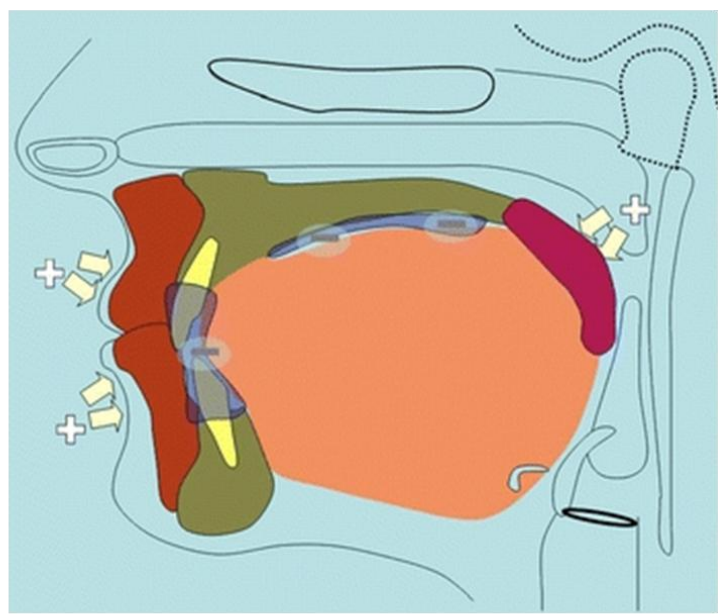

B. Geschlossene Ruhelage nach dem ZRM

Abbildung 6 Einfluss des Zungenrepositionsmanövers (ZRM) auf das biofunktionelle Modell (Engelke et al. 2011, Seite 168)

In der Abbildung 6 wird eine schematische Darstellung des biofunktionellen Modells gezeigt. Im Schema A wird das Bestehen von negativen Drücken in den Kompartimenten 1 und 2 beim forcierten Schluckvorgang gezeigt. Extraoral herrscht der atmosphärische Druck. Die roten Pfeile weisen auf neuromuskuläre Aktivität hin. Im Schema B wird die geschlossene Ruhelage dargestellt, die durch die gebildeten Verschlüsse V1, V2 und V3 gekennzeichnet ist. 


\subsection{Physiologie und Pathologie des Schluckaktes}

Schlucken ist ein komplexer physiologischer Mechanismus, der die Vorbereitung der Speise und deren Transport zum Magen sichert. Dazu ist eine intakte Koordinierung mit der Atmung notwendig (Matuso und Palmer 2009). Meist bleibt der Schluckvorgang unbeachtet, indem der im Mund gesammelte Speichel zwischen den Mahlzeiten und im Schlaf geschluckt wird. Nach etwa $0,5 \mathrm{ml}$ Speichelakkumulation wird der Schluckakt ausgelöst, was eine Anzahl von 800 bis zu 1200 Schluckvorgängen am Tag mit einer Frequenz von ungefähr einem Schluckvorgang pro Minute bedeutet (Rakosi 1970; Dodds et al. 1990).

Die neurologische Regulation des Schluckaktes erfolgt in einem großen Areal des Hirnes, besonders im sensomotorischen Kortex, der Insula und dem temporopolaren Kortex, im Hirnstamm sowie im Kleinhirn. Die Repräsentationsareale für das Schlucken sind im Kortex meist asymmetrisch, dementsprechend ist eine Großhirnhemisphäre schluckdominant. Die breite Verteilung und Beteiligung der verschiedenen Hirnareale könnte eine Erklärung für die hohe Variabilität der Schluckstörungen sein (Hamdy et al. 1999). Die motorischen Hirnnervenkerne des efferenten Systems, die sensorischen Fasern der Hirnnerven des afferenten Systems und die „Central Pattern Generators“ an der Medulla oblongata sind hier von Bedeutung (Böhme 2003; Prosiegel et al. 2005).

Für einen koordinierten und geschützten Schluckakt sorgen fünf Hirnnerven und drei zervikale Nerven (C1-3). Zu den beteiligten Hirnnerven gehören der N. trigeminus (V), der die Kaumuskulatur innerviert, der N. facialis (VII), der die mimische Muskulatur innerviert, der N. glossopharyngeus (IX) innerviert die Pharynxmuskulatur und sorgt für die sensible Innervation des hinteren Zungendrittels, der Gaumenbögen und des oralen Anteils des Pharynx (Böhme 2003). Zentral wird der Schluckakt von der Medulla oblongata gesteuert. Erreicht der Bolus die Triggerzonen, die sich an den Gaumenbögen, dem Zungengrund und der Rachenhinterwand befinden, wird der Schluckakt reflektorisch ausgelöst. Der Impuls wird von den afferenten sensorischen Fasern des N. glossopharyngeus und N. vagus zum Reflexzentrum geleitet (Lang 2009). 


\subsubsection{Schluckphasen}

Zur vereinfachten Darstellung und um den Schluckakt besser zu verstehen, wird dieser in Phasen unterteilt. Es handelt sich jedoch um einen kontinuierlichen Prozess. Eine der bekannten Klassifikationen unterscheidet eine willkürliche und eine unwillkürliche Phase. Zur willkürlichen Phase gehören die Vorbereitung der Speise zu einem geeigneten Bolus und dessen Transport zur Triggerzone. Ab diesem Punkt wird der Bolustransport vom Oropharynx bis zum Magen durch einen unwillkürlichen Mechanismus übernommen (Böhme 2003).

Eine weitere Differenzierung in drei Schluckphasen wurde 1813 erstmalig von Magendie vorgeschlagen. Er unterteilte den Schluckakt in eine orale, eine pharyngeale und eine ösophageale Phase (Magendie 1813). Eine vierte Phase, die Vorbereitungsphase, wurde von Logemann (1988) zu der von Magendie beschriebenen Klassifikation hinzugefügt (Logemann 1988; Logemann 1998).

\subsubsection{Die Vorbereitungsphase}

Diese Phase beginnt mit der Aufnahme der Nahrung und endet mit dem für den Schluckakt fertig präparierten Bolus. Abhängig von der Nahrungsart ist die Aufnahme durch das Beißen einer festen Speise, durch das Einfließen einer Flüssigkeit oder das Ansaugen mit einem Strohhalm möglich. Sobald sich die Speise bzw. Flüssigkeit in der Mundhöhle befindet, wird sie entsprechend der Konsistenz verarbeitet. Bei fester Nahrung gewinnt die Kaufunktion an Bedeutung, damit die Speise zerkleinert wird (Orchardson und Cadden 2009). Die Zunge führt den Bolus auf die Kauflächen, mischt und befeuchtet ihn mit Speichel. Hierbei führt der Unterkiefer Öffnungs- und Seitwärtsbewegungen aus. Die Kaumuskulatur ist aktiv beteiligt und die Lippen sind geschlossen (Logemann 1988). Die Dauer dieser Phase ist von Genuss und persönlichen Angewohnheiten abhängig (Wendler et al. 2005). Bei flüssiger Kost ist diese Phase deutlich kürzer, da keine Modifikation zu einem adäquaten Bolus notwendig ist.

\subsubsection{Die orale Phase}

In dieser Phase wird der fertig vorbereitete Bolus mit koordinierten Zungenbewegungen den Triggerzonen präsentiert. Sollte sich der Bolus nach der Vorbereitungsphase bereits auf dem Zungenrücken befinden, dann handelt es sich nach radiologischen 
Untersuchungen von Arbeitsgruppen Cook et al. (1989) und Dodds et al. (1989) um einen „Schneidezahntyp“. Wenn der Bolus im vorderen Mundbodenbereich liegt, bezeichnet man das als „Schöpflöffeltyp“. In beiden Fällen wird der Bolus bis zum Bereich der Gaumenbögen geführt. Nach der Berührung dieses Areals und der oropharyngealen Hinterwand wird der Schluckreflex ausgelöst. In röntgenologischen Aufzeichnungen konnte beobachtet werden, dass sich hierbei die Verbindung des Velums mit dem Zungengrund löst und der Bolus in den Pharynx gelangen kann (Dodds et al. 1990). Damit wird die orale Phase beendet. Die Dauer der oralen Phase ist von der Boluskonsistenz abhängig und dauert in der Regel weniger als eine Sekunde (Böhme 2003).

\subsubsection{Die pharyngeale Phase}

Die pharyngeale Phase gehört zu den unwillkürlichen Phasen des Schluckens und ist von wichtigen neuromuskulären Ereignissen geprägt (Robbins 1996). Eine nasale Regurgitation wird durch den Verschluss des Naseneinganges verhindert. Dieser wird vom Velum, dessen Kontakt zur pharyngealen Hinterwand und der Aktion der Konstriktoren realisiert (Foucart et al. 1998). Die Elevation des Larynx und des Hyoids verhindern den Boluseintritt in den laryngealen Luftweg. Nach dieser Hebung stößt der Larynx gegen den Zungengrund und es resultiert ein Larynx-, Taschenfalten-, und Stimmlippenverschluss. Die Ary-Region nähert sich der laryngealen Fläche der Epiglottis und die Zungenbasis zieht sich ein (Böhme 2003). Der Bolus erreicht in diesem Moment die Valleculae und den Sinus piriformis. Die krikopharyngeale Region wird extrinsisch gestreckt. Durch die Elevation des Pharynx wird der ösophageale Sphinkter geöffnet und der Bolus tritt in den Ösophagus ein (Böhme 2003). Die pharyngeale Phase dauert in der Regel eine Sekunde (Atkinson et al. 1957).

\subsubsection{Die ösophageale Phase}

Die letzte Phase des Schluckens ist von peristaltischen Bewegungen gekennzeichnet. Es handelt sich um unwillkürliche Kontraktionen, die in zwei Wellen von kranial ausgehend nach kaudal stattfinden, um den Bolus zu mobilisieren (Hendrix 1993). Unterdrücke, die einen Sog bilden, begleiten diese Phase und spielen eine wichtige Rolle (Blonski et al. 2008). Am Mageneingang befindet sich der untere ösophageale Sphinkter. Nach dessen Öffnung gleitet der Bolus in den Magen hinein. Zur gleichen 
Zeit senken sich der Larynx und das Hyoid, die Epiglottis richtet sich wieder auf. Die ösophageale Phase ist langsamer und kann sechs bis zwölf Sekunden dauern. Mit ihr ist der Schluckvorgang beendet (Böhme 2003; Logemann 1988; Prosiegel und Weber 2009).

Eine schematische Darstellung der Schluckphasen ist in der Abbildung $7 \mathrm{zu}$ sehen. Da die Vorbereitungsphase stark variiert und individuell abhängig ist, wurde sie nicht in der Abbildung dargestellt.
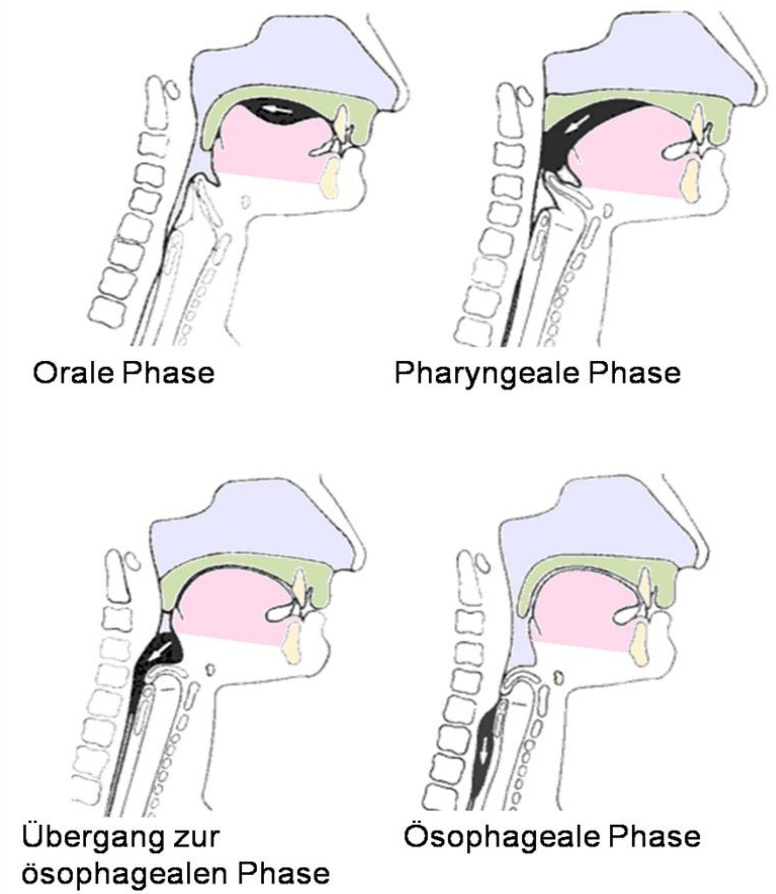

Abbildung 7 Verlauf der Schluckphasen bei einem normalen Schluckakt (Modifiziert nach Logemann 1998, Seite 28)

\subsubsection{Entwicklung der oralen Saugschluckfunktion}

Beim Säugling verläuft die Schluckfunktion reflektorisch, mit dem lebensnotwendigen Ziel, Milch aus der Brust zu gewinnen. Dieser Reflex findet schon beim Feten statt, indem Fruchtwasser geschluckt wird (Rakosi 1970). Der genaue Mechanismus, wie Säuglinge Milch aus der Brust gewinnen, ist noch umstritten. Bisher wurde über eine repetitive Kompression der Brustwarze zwischen den Kieferkämmen, peristaltischen 
Bewegungen der Zunge (Wein et al. 1993) und über die Erzeugung von einem intraoralen Vakuum diskutiert (Geddes et al. 2008).

Das beim Säugling physiologische infantile Schlucken wird auch viszerales Schluckmuster genannt. Dieses Muster ist von einer anterioren Lage der Zunge ausgezeichnet, die zwischen beiden Kieferkämmen liegt. Die Zungenspitze bildet mit den Lippen einen Zungenlippenkontakt (Rakosi 1970). Die Stabilisierung des Unterkiefers während des viszeralen Schluckens wird durch die Aktivität der perioralen Muskulatur und der Wangenmuskulatur unterstützt (Moyers 1973). Im Laufe der Zeit entwickelt sich der Schluckakt von einem infantilen Schluckmuster beim Säugling zu einem somatischen. In der Zwischenzeit kann ein Übergangsschluckmuster auftreten (Graber 1963).

Physiologisch bleibt das viszerale Schluckmuster etwa bis zum sechsten Lebensmonat erhalten, bis der Durchbruch der Milchzähne stattfindet (Lavelle 1957). Dieser Moment stimmt auch mit einer Änderung der Nahrung überein, da die bisher alleinige Milchnahrung durch breiartige Speisen ergänzt wird. Der Durchbruch der Frontzähne verhindert die anteriore Zungenlage zwischen den Kieferkämmen. Im posterioren Bereich passiert dies mit der Eruption der Milchmolaren. Im normalen Fall verändert sich hierbei das Schluckmuster, die Zunge befindet sich während des Schluckaktes hinter den Zahnreihen und berührt die Papilla incisiva. Die Stabilisierung des Unterkiefers wird von den Schließmuskeln übernommen, die periorale Muskulatur bleibt daher beim Schlucken entspannt (Lavelle 1957).

\subsubsection{Habituelle Schluckstörungen}

Die ersten Beobachtungen von Schluckvariationen wurden im Zusammenhang mit Malokklusionen durchgeführt (Rix 1961). Eine „,pervertierte Schluckweise“ wurde 1951 von Straub beschrieben. Bei den von ihm untersuchten Kindern konnte er feststellen, dass sich die Zunge in der Ruhelage zwischen die Frontzähne schob. Beim Schlucken beobachtete er auch, dass ein Grimassieren der Lippen auftrat und ein positiver Druck im Mundraum generiert wurde (Straub 1951). 
Habituelle Schluckstörungen können unterschiedliche Teilfunktionen betreffen und sich durch manuelle Interaktionen, Körperhaltungsanomalien oder die Benutzung von Gegenständen manifestieren (Friedrich und Bigenzahn 1995).

Gekennzeichnet wird das persistierende viszerale Schluckmuster von den Bewegungen der Zunge und der perioralen Muskulatur. Die Zunge kann gegen die Frontzähne drücken oder zwischen ihnen liegen. Auch die Zungenränder können gegen oder zwischen die Zahnreihen gepresst werden. Wichtig ist, dass die Zungenmitte nicht den Gaumen berührt, sondern an den Zähnen oder am Mundboden liegt (Kittel 2001). Der Muskeltonus der Zunge ist oft unausgeglichen, das heißt, die Zungenmitte ist meist schwach, der Zungenrand hingegen verstärkt angespannt (Kittel 2001). Die Zungenbewegungen, die beim viszeralen Schluckmuster in sagittaler Richtung durchgeführt werden können, sind verschieden und zahlreich (Schwestka-Polly et al. 1992). Bei Patienten mit offenem Biss kann es sich auch um vertikale und Ab- und Vorwärtsbewegungen handeln (Peng et al. 2003).

Im Gegensatz zum somatischen Schlucken ist beim viszeralen Schluckmuster extraoral eine Aktivität der Lippen-, Wangen- und Kaumuskulatur sichtbar (Gross et al. 1990). Die Lippen werden beim viszeralen Schlucken stark gepresst, somit sind sie manuell nicht zu öffnen. Hierbei tritt häufig eine dystonische Oberlippe mit erhöhter Aktivität des M. mentalis auf. Der M. mentalis zieht die Unterlippe bis zum Kontakt mit der Oberlippe nach kranial (Kydd et al. 1963). Der Schluckakt wird oft von einer anterioren Kopfbewegung und einer extraoral sichtbaren Aktivität der mimischen Muskulatur begleitet (Straub 1951).

\subsubsection{Organische Schluckstörungen}

Eine pathologische Alteration des Schluckmechanismus wird Dysphagie genannt und kann einzelne oder mehrere Phasen des Schluckaktes in verschiedenem Ausmaß betreffen (Böhme 2003). Auffällig werden Schluckstörungen durch Dehydratation, Verlust an Körpergewicht und Verschlucken während der Aufnahme von Nahrung oder Flüssigkeit. Beim Verschlucken entsteht das größte Risiko für eine Aspiration (van der Maarel-Wierink et al. 2011). 
Ursachen für Dysphagien sind am häufigsten neurogen bedingte Erkrankungen oder Strukturveränderungen nach einer Tumorresektion im orofazialen Bereich. Andere Ursachen können angeborene Einschränkungen der oralen Phase sein, wie bei neurogenen Zerebralparesen oder strukturellen Defekten (z.B. Lippen- Kiefer-GaumenSpalte). Seltenere Ursachen für erworbene Störungen des Schluckens sind Zustände nach Zahnverlust, insuffiziente Zahnprothesen oder ein medikamentös sekundär reduzierter Speichelfluss (Böhme 2003; Redford-Badwal et al. 2003).

\subsubsection{Entwicklungsbedingte neurogene Schluckstörungen}

Eine neurogene Dysphagie kann konnatal präsent sein, wenn eine neurologische Erkrankung vorliegt. Hierbei liegt die Ursache meistens in einer infantilen Zerebralparese (Love et al. 1980), bei der besonders die orale und die pharyngeale Phase stark beeinträchtigt werden (Otapowicz et al. 2010). Andere neurogen bedingte Störungen im perinatalen Alter können als Folge eines fetalen Alkoholsyndroms (Prasse und Kikano 2008) und bei einer Frühgeburt auftreten. In diesen Fällen ist die Dysphagie durch ein unreifes Reflexmuster beim Schlucken bedingt, das nach einigen Wochen kompensiert wird (Tuchman 1988; Bootz 1995; Jensen et al. 2003).

\subsubsection{Erworbene neurogene Schluckstörungen}

Erworbene neurogene Dysphagien können auf verschiedene Schädigungen zurückgeführt werden. Im Laufe des Kindesalters können verschiedene Ereignisse wie Hypoxien, Anoxien, neurodegenerative Erkrankungen, Infektionen des zentralen Nervensystems und Schlaganfälle stattfinden, die als Folge eine Dysphagie hervorrufen können (Christensen 1989). Bei Erwachsenen können auf gleiche Art fokale Hirnverletzungen des Schluckkortex und Hirnstammes zu neurogenen Dysphagien führen. Nach einer Studie von Prosiegel et al. (2002) gehören neurogene Verletzungen mit circa 50\% zur häufigsten Ursache bei neurogener Dysphagie (Prosiegel et al. 2002). Hierbei handelt es sich meistens um Hirninfarkte oder Hirnblutungen (Kuhlemeier 1994; Prosiegel et al. 2002). Dass eine neurogene Dysphagie nach einem Schlaganfall entsteht, wird bei einem Drittel der betroffenen Patienten beobachtet (Smithard et al. 1997), in akuten Fällen sogar bei $80 \%$ der Patienten (Suntrup et al. 2012). Weitere neurogene Ursachen sind unter anderem: schwere Schädel-Hirn-Traumata, Hirntumoren, pseudobulbäre Paresen, neurodegenerative und multisystemische 
degenerative Erkrankungen wie Morbus Parkinson und Multiple Sklerose (Prosiegel et al. 2004; Cook 2009; Restivo et al. 2011).

Bei einer neurogenen Dysphagie ist die Koordination, der beim Schlucken benötigten Muskeln sowie das Auslösen des Schluckreflexes beeinträchtigt. Besonders betroffen sind die orale und pharyngeale Phase (Otapowicz et al. 2010). In der oralen Phase spielt die verlorene Zungenkontrolle eine wichtige Rolle (Lazarus und Logemann 1987), was sich in einer eingeschränkten Bolusführung und einem erhöhten Risiko der zu frühen Boluspassage in den Pharynx äußert (Logemann 1985). Diese pathologische Aktivität der Zunge ist auch mit einer verlängerten oralen Transitzeit verbunden (Field und Weiss 1989).

\subsubsection{Angeborene strukturell bedingte Schluckstörungen}

$\mathrm{Zu}$ morphologischen Defiziten, die mit einer Dysphagie als Folge auftreten können, gehören angeborene Defekte wie die Lippen-Kiefer-Gaumen-Spalte (Choi et al. 1991; Reid et al. 2007). Bei einem geringeren Defekt, der isoliert nur einen Teil der intraoralen Strukturen betrifft, werden kompensatorische Maßnahmen ergriffen, wie zum Beispiel bei einer Lippenspalte. In diesen Fällen kann der äußere Verschluss der Mundhöhle erschwert werden und es besteht das Risiko einer extraoralen Leckage; meist ist aber die Nahrungsaufnahme trotzdem möglich (Shah und Wong 1980). Bei einer fehlerhaften Entwicklung des harten und weichen Gaumens entsteht eine Verbindung zur Nasenhöhle, welche das Schlucken stark behindert (Cooper-Brown et al. 2008). In diesen Fällen ist eine prothetische Versorgung des Defekts und der Einsatz von Spezialschnullern und komprimierbaren Trinkflaschen notwendig, um die frühe Ernährung zu ermöglichen (Reid 2004; Masarei et al. 2007). Gemäß dem Wachstum des betroffenen Kindes folgen operative Korrekturen, die im Rahmen einer multidisziplinären Behandlung - wie zum Beispiel mit einer kieferorthopädischen und phoniatrischen Therapie - erfolgen müssen, um so eine definitive Korrektur des

Defektes zu erreichen (Habel et al. 1996; Sánchez-Ruiz et al. 1999; Pearson und Kirschner 2011).

\subsubsection{Erworbene strukturell bedingte Schluckstörungen}

Erworbene strukturelle Störungen, die eine Dysphagie verursachen können, werden beispielsweise durch vorübergehende entzündliche Zustände verursacht oder können 
eine bedeutendere Pathogenese haben: dazu zählen Tumore der Mundhöhle, des Oropharynx, des Hypopharynx und der Supraglottis und deren folgende therapeutische Maßnahmen (Jäghagen et al. 2008). Hypopharynxdivertikel, extrapharyngeale Kompressionen, Verätzungen und Systemerkrankungen wie Amyloidose oder Sklerodermie können ebenfalls Schluckstörungen hervorrufen (Motsch 2005).

In Bezug auf die oben genannten strukturellen Ursachen kommen Tumoren im orofazialen Bereich am häufigsten vor. Kopf- und Hals-Tumoren haben laut der aktuellen GLOBOCAN-Studie (2008) eine Inzidenz von 4.6\% (Ferlay et al. 2010) und verteilen sich abnehmend von Karzinomen in der Mundhöhle, über Larynx und Oropharynx zu seltener auftretenden Nasopharynx-Karzinomen (Parkin et al. 2001). Tumore im oropharyngealen Bereich korrelieren stark mit Risikofaktoren wie Tabakund exzessivem Alkoholgenuss (Wynder et al. 1957; Blot et al. 1988). Auch virale Infekte können ein Risikofaktor sein, z.B. Epstein-Barr-Virus oder der humane Papilloma-Virus (Dictor und Johnson 2000). Ein gastroösophagealer Reflux (El Serag et al. 2001) und diätetische Aspekte (McLaughlin et al. 1988) spielen ebenfalls eine Rolle.

Ein Tumor im oropharyngealen Bereich kann eine Dysphagie durch seine Ausdehnung auslösen. Ebenfalls kann eine Schluckstörung durch die therapeutischen Maßnahmen bedingt sein. Dazu zählen je nach Tumorart die Resektion, die Rekonstruktion, Radiound Chemotherapie (Suarez-Cunqueiro et al. 2008). In der Regel kann man sagen, je größer die Resektionsfläche ist, desto stärker kann die Schluckfunktion betroffen sein (McConnel et al. 1994). Besonders nach der Resektion eines Zungenanteils werden Schluckstörungen häufig beobachtet (Pauloski et al. 2004). Außer aus den resultierenden Weichteil- und Knochendefekten spielen auch der chirurgische Zugang und die Rekonstruktionsmethode eine wichtige Rolle. Dementsprechend können die Motorik, die Sensibilität und Innervation nach einer Tumorresektion geschädigt werden (Mittal et al. 2003). Die nach der Tumorresektion oft vorgesehene Radio- und Chemotherapie trägt ebenso $\mathrm{zu}$ einer Verschlechterung des Schluckvorgangs bei (Gaziano 2002). Hier ist mit dem Auftreten von Fibrosen und Schrumpfungen des Gewebes zu rechnen (Mittal et al. 2003), sowie mit einer Reduktion des Speichelflusses, sensorischen Missempfindungen (Lazarus 1993) und einer Parese der Stimmbänder (Wu et al. 2000). 


\subsection{Diagnostik und Beurteilung des Schluckaktes}

$\mathrm{Zu}$ einer zuverlässigen Betrachtung des Schluckaktes sind Anamnese sowie klinisch und technisch gestützte Verfahren notwendig. Auch Kenntnis über systemische Grunderkrankungen und medikamentöse Therapien müssen für eine korrekte Beurteilung des Schluckaktes miteinbezogen werden.

\subsubsection{Klinische Anamnese}

Nach Schröter-Morasch (1994) gehören zum klinischen Befund Fragestellungen über Schluckbeschwerden, Husten und Lokalisation der Symptome. Weiter zu beachten sind das Auftreten von „Globus pharyngis“ (Kloß-Gefühl) und Stimm-, Sprech- und Sprachstörungen (Schröter-Morasch 1994).

Eine klinische Untersuchung der Schluckfunktion wird unternommen zur Beurteilung folgender Aspekte: Nahrungsaufnahme, Mundschlussbildung, Kauvermögen, Schluckdauer, Schluckreflexauslösung, Bewegungen des Kehlkopfes und Zungenbeins, Effektivität des Schluckvorgangs, Auftreten von Leitsymptomen einer Aspiration wie Husten und Erstickungsanfälle, Änderung der Stimmqualität (Wendler et al. 2005).

\subsubsection{Diagnostische Verfahren}

Bei der Untersuchung des Schluckaktes sind bildgebende Verfahren von großer Bedeutung, die den Schluckverlauf räumlich und zeitlich darstellen. Untersuchungen mit einer schnellen Auflösung können zur Diagnostik einer Schluckstörung oder einer unauffälligen Aspiration beitragen.

\subsubsection{Videofluoroskopie}

Die Videofluoroskopie bezeichnet eine in hoher Geschwindigkeit ablaufende röntgenologische Aufzeichnung des Schluckvorganges. Als Kontrastmittel wird normalerweise Barium genutzt, welches mit Flüssigkeiten oder Nahrung verschiedener Konsistenz gemischt werden kann. Mit dieser Methode können dynamische Änderungen während des Schluckens erkannt werden. Der Bolus-Transit und die Bewegungen der pharyngealen Strukturen können während der Schluckphasen 
überprüft werden (Logemann 1993; Kahrilas et al. 1997). Eine Stauung des Bolus oder das Eindringen in die unteren Atemwege im Falle einer Aspiration kann hiermit untersucht werden. Obwohl diese Methode als Standard in der Schluckdiagnostik etabliert ist, sind einige Nachteile zu beachten, wie zum Beispiel die Strahlenbelastung, die Einnahme von Kontrastmitteln und der Bedarf einer radiologischen Ausstattung (Logemann et al. 1998).

\subsubsection{Videoendoskopie}

Eine Alternative zur Videofluoroskopie ist die Videoaufzeichnung durch eine endoskopische Untersuchung des Pharynx (Videoendoskopie). Hier wird eine flexible Optik nasal zum Pharynx geführt und der Schluckakt in vivo aufgezeichnet (Langmore et al. 1991). Abhängig von der Höhe des Endoskops, können verschiedene Etagen des Pharynx beim Schlucken beurteilt werden. Anatomisch erlaubt dieses Verfahren eine Untersuchung des nasalen und pharyngealen Weichgewebes, was auch für einen Rezidivausschluss nach Tumorresektionen in diesem Bereich relevant ist (Simental und Carrau, 2004). Funktionell werden während des Schluckaktes das Verbleiben von Bolusresten oder die Ansammlung von Sekreten überprüft. Abnorme Bewegungen des Pharynx, des Larynx und der Stimmlippen können ebenso beurteilt werden. Vorteile dieses Verfahrens sind die qualitativen Beurteilungen des Schluckvorganges, die Möglichkeit, diese Untersuchung am Bett des Patienten durchführen zu können, und die geringen Nebenwirkungen (Hiss und Postma, 2003). $\mathrm{Zu}$ den Nachteilen zählen die fehlende Auskunft über die orale und pharyngeale Phase. Die orale Phase wird überhaupt nicht untersucht und der Pharynx kann nur vor und nach der Bolus-Passage dargestellt werden (Logemann et al. 1998).

Videofluoroskopie und Videoendoskopie gehören $\mathrm{zu}$ den etablierten schluckdiagnostischen Verfahren bei dysphagischen Patienten. Nach einer Studie von Aviv (2000) konnte festgestellt werden, dass beide Untersuchungen einen hohen Stellenwert bei der Diagnose von Aspiration haben. Entscheidend sind der Kostenaufwand, die Kooperation der Patienten und die Fertigkeit des Untersuchers bei der Interpretation der Ergebnisse (Aviv 2000). 


\subsubsection{Elektromyographie}

Da der Schluckakt muskelabhängig verläuft, ist eine elektromyographische Untersuchung hilfreich, bei der eine individualisierte Muskelaktivität gemessen werden kann. Hierbei ist eine zeitliche Differenzierung zwischen kontrahierten und relaxierten Muskelzuständen möglich (Palmer 1989). Wertvoll ist diese Untersuchung bei der gezielten Identifizierung von neuromuskulären Ursachen einer Schluckstörung und für die Differenzialdiagnose zwischen einer Dysphagie und einer Odynophagie. Mangelhaft ist diese Untersuchungsmethode bei der Diagnose einer neurogenen Dysphagie (Vaiman und Eviatar 2009).

\subsubsection{Akustische Verfahren}

Die akustische Auswertung des Schluckvorganges basiert auf der zervikalen Auskultation, die normalerweise mit einem Stethoskop erfolgt. Hierbei werden pharyngeale Geräusche wie zum Beispiel die der Epiglottis während des Schluckens abgehört (Santamato et al. 2009). Diese Laute können vom Untersucher klinisch ausgewertet oder für eine Vergleichskontrolle aufgenommen werden. Vorteilhaft bei diesem einfachen und kostengünstigen Verfahren ist, dass Dysphagien und Aspirationen leicht festgestellt werden können. Trotzdem kann die Auskultation aufgrund limitierter Aussagekraft nur als adjuvante Technik betrachtet werden (Cichero und Murdoch 2002).

\subsubsection{Magnetresonanztomografie (MRT)}

Bei dieser Untersuchung wird der Schluckakt und dessen Verlauf mit einer schnellen bildgebenden Auflösung gezeigt ohne Nebenwirkungen oder Risiken für den Patient. Sowohl Weichteilgewebe als auch der Bolus können mit dieser Methode dargestellt werden. Die MRT-Untersuchung erlaubt eine genaue Beurteilung der Zungenbewegungen, des weichen Gaumens und anderer beteiligter Strukturen während des Schluckens (Breyer et al. 2009). Verhindert wird der massive Einsatz dieser Technik durch die hohen Kosten.

\subsubsection{Palatographie}

Dieses Verfahren wird verstärkt bei Sprachstörungen eingesetzt. Es werden mittels Verfärbungen die Stellen im Mundraum sichtbar, wo Zunge und Gaumen in Kontakt 
getreten sind. Dieses Verfahren stellt kein biologisches Risiko für die Patienten dar und beeinflusst den normalen Verlauf des Schluckvorganges nicht (Engelke et al. 1990). Ein Nachteil ist, dass keine Aussage über den temporären Ablauf der Zungenbewegungen getroffen werden kann. Eine Variation der Palatographie ist mit der Elektropalatographie gegeben, bei der keine Farben zum Einsatz kommen, sondern die Mundraumkontakte über Elektroden bestimmt werden (Hardcastle et al. 1991; Hiiemae und Palmer 2003).

\subsubsection{Sonographie}

Der Ultraschall ist ein diagnostisches Verfahren, das mittels Schallwellen und deren Reflexion, eine Beurteilung der Position von Strukturen in einer Zeitachse erlaubt. So werden qualitative und quantitative Analysen der Zungenbewegungen durchgeführt, ohne in den intraoralen Raum einzudringen. Die Sonographie ist eine Methode, die bei Kindern und Erwachsenen mit verminderter Compliance leicht anzuwenden ist (Böcker et al. 1989).

Mit dieser Technik könnem die Zungenmuskulatur und ihre Bewegungen besonders beobachtet werden. Begrenzt ist dieses Verfahren, wenn eine umfassende Beurteilung des Schluckaktes im oralen, pharyngealen und laryngealen Bereich angestrebt wird (Chi-Fishman et al. 1998; Chi-Fishman 2005).

\subsubsection{Elektromagnetische Artikulographie (EMA)}

Mit der elektromagnetischen Artikulographie ist es möglich, elektrische Potenziale der Muskeln abzuleiten, indem an der Oberfläche der Muskeln Elektroden befestigt werden. Im Falle des Schluckaktes werden Stärke und Dauer der Muskelaktivität bestimmt (Engelke et al. 1996). Ein besonderer Vorteil dieser Technik ist die hohe Auflösung und die präzise Untersuchung der Muskeln sowie das Fehlen von Bestrahlung oder Kontrastmittel. Die Anwendung dieser Methode ist aber aufgrund des hohen Aufwands und der nicht möglichen Abbildung von Bolus und benachbarten Strukturen begrenzt (Steele und Van Lieshout 2004).

\subsubsection{Druckmessungen}

Der Mechanismus, mit dem der Bolus den Mund verlässt und in den Pharynx gelangt, wird traditionell als eine Wirkung der Zunge gegen den Gaumen beschrieben (Steele 
und Van Lieshout 2009). Diese Zungenkraft wird mittels Druckmessungen quantifiziert - ein Verfahren, das sich als wichtige Untersuchung bewährt und etabliert hat (McConnel et al. 1988a).

Zur manometrischen Diagnose sind verschiedene Alternativen möglich, die sich im Laufe der Zeit und gemeinsam mit technischen Fortschritten entwickelt und differenziert haben. Ein wichtiger Aspekt bei Druckmessungen ist einerseits die Entscheidung, ob der Kontaktdruck zwischen verschiedenen Strukturen oder der Kompartimentdruck in einem Raum Ziel der Messung ist. Andererseits besteht die Frage, ob es sich um kieferorthopädische oder um schluckphysiologische Untersuchungen handelt.

Die wichtigsten Komponenten bei der manometrischen Interpretation des orofazialen Systems sind der Kauapparat und die Zunge, wobei diese beiden Elemente interagieren und in enger Beziehung zueinander stehen (Ardran und Kemp 1951; Weinstein et al. 1963). Genauere Aspekte der Interaktionen und mögliche Wirkungen zwischen Zunge und Zahnreihe sind heutzutage noch umstritten (Tomes 1873; Proffit 1978). Wichtig beim Schluckakt ist die Erkenntnis, dass die Zunge eine dominierende Rolle bei der Bildung, Positionierung und Manipulation des Bolus sowie bei dessen Transport in den Pharynx spielt (Magendie und Revere 1855; Dodds 1989).

Die erste bekannte Untersuchung zur oralen Druckmessung während der Kaufunktion stammt aus Italien und wurde für die Messung der Muskelkraft bei Bewegungen des Unterkiefers 1681 von Borelli angewendet. Diese Messung ist bekannt unter dem Namen „Gnathodynamometer“. Sie erfolgte extraoral, indirekt und stützte sich auf die Anwendung eines einfachen Aufbeißhebels. Weitere Gnathodynamometer, basierend auf Spiralfedern, wurden später auch zur Kaukraftmessung von Haber 1926 und 1927 angewendet (Brawley und Sedwick 1938).

Eine Perfektionierung dieses Systems und dessen intraorale Anwendung konnte mit der Einführung von Dehnungsmessstreifen erreicht werden. Das Prinzip der Dehnungsmessstreifen beruht auf Drücken, die den Dehnungsstreifen verformen. Die Verformungen lösen eine Änderung des elektrischen Widerstands aus, die als Druckänderungen interpretiert werden können. Dieses Phänomen wurde bereits 1856 von Lord Kelvin entdeckt (Window 1992) und 1938 als Druckmessgerät von Simmons und Ruge - unabhängig voneinander - erfunden (Hesse und Schnell 2004). 
Die Anwendung von Dehnungsmessstreifen bei intraoralen Druckmessungen verblieb lange Zeit im kieferorthopädischen Bereich und wurde erstmals 1948 von Howell und Manly genutzt. Hierbei haben die Wissenschaftler besonders auf die Dimension des Gerätes geachtet, da die vorherigen Druckmesssysteme ein sehr großes Volumen aufwiesen (Howell und Manly 1948). Da diese Messmethode sich als erfolgreich erwies, wurde sie erweitert und für die Messung der Kaukraft angewendet (Howell und Brudevold 1950). Einige Jahre später verbreitete sich diese Technik und wurde von anderen Wissenschaftlern übernommen (Winders 1956). So untersuchte Kydd im Jahre 1956 die Kraft der Zunge gegen den Zahnbogen (Kydd 1956). Auch Proffit et al. nutzten dieses Verfahren, um die Zungenfunktion und ihren Einfluss auf den Zahnbogen im Wachstum zu erforschen (Proffit et al. 1969). Später wendeten sie das System an, um die Entwicklung vom infantilen zum somatischen Schluckakt zu prüfen (Proffit 1972). Winders verwendete Dehnungsmessstreifen bei seinen SchluckaktUntersuchungen im Jahre 1958 und stellte dabei fest, dass bei normalen okklusalen Verhältnissen die Lippen und Wangenmuskulatur während des Schluckakts nicht kontrahierten und dass die Zungenspitze sich lingual der oberen Schneidezähne befand. Er konnte auch erkennen, dass die Zunge eine signifikant stärkere Kraft gegen den Zahnbogen während der Funktion ausübte als die periorale Muskulatur (Winders 1958). Diese Ergebnisse wurden etwas später von Gould und Picton 1964 bestätigt, als sie verschiedene orale Funktionen mit Dehnungsstreifen untersuchten (Gould und Picton 1964).

Eine der ersten Bemühungen, den Schluckakt unabhängig von der Kaukraft genauer zu erfassen, führt ins Jahr 1966 zurück, als Lear und Morrees (1966) mit einem sogenannten „frequency modulated telemetry system“ akustische Langzeitmessungen des Schluckvorganges registrierten. Vier Jahre später ergänzten sie diese Methode mit Dehnungsmessstreifen und konnten so die Kraft der perioralen und lingualen Muskulatur auf den Zahnbogen beim Schlucken messen. Obwohl diese Methode weiterhin kieferorthopädische Fragen beantworten sollte, konnten hierbei die ersten manometrischen Daten zur Schluckfunktion extrapoliert werden (Lear und Moorrees 1969).

Eine der ersten rein manometrischen Schluckuntersuchungen wurde 1988 von Shaker et al. durchgeführt. Auch hier wurden Dehnungsmessstreifen genutzt, die an verschiedenen intraoralen Punkten - sub- und supralingual - positioniert wurden. Ziel 
dieser Untersuchung war es, Druckgradienten während des Schluckvorganges zu registrieren. Gekoppelt wurden diese Messungen mit videoröntgenologischen Aufzeichnungen. Mit dieser Versuchsanordnung konnten Druckänderungen während der oralen Phase des Schluckens festgestellt werden, die zeitlich und anatomisch korrelierten (Shaker et al. 1988).

Ein alternatives Messverfahren zum Dehnungsmessstreifen wurde von Robin et al. im Jahre 1992 präsentiert. Hierbei handelte es sich um einen luftgefüllten Bulbus. Dieser wurde intraoral positioniert und extraoral an einen Drucksensor angeschlossen (Robin et al. 1992). Dieses Gerät, „Iowa Performance Instrument (IOPI)“ (IOPI Northwest ${ }^{\circledR}$, Carnation, WA) genannt, wurde von verschiedenen Wissenschaftlern zur Schluck- und Sprechdiagnostik verwendet (Robbins et al. 1995; Lazarus et al. 2000; Vitorino 2010) und wird heute noch als Diagnostik- und Übungsgerät eingesetzt. Genauere Informationen zum Schlucken können mit den "Kay Elemetrics" (Kay Elemetrics Corp ${ }^{\circledR}$, Lincoln Park, NJ) gewonnen werden. Dieses Gerät gehört heutzutage zu den meistverwendeten Druckmesssystemen mit kombinierten manometrischen, elektromyographischen und endoskopischen Aufzeichnungen. Dabei erfolgt die Manometrie mittels luftgefüllter Bulbi, die intraoral platziert werden können (Hind et al. 2005; Ozgursoy und Salassa 2010). Im Allgemeinen wurde der Druck der Zunge gegen Strukturen im Mundraum gemessen. Änderungen des intraoralen Kompartimentdruckes wurden von den meisten Arbeitsgruppen nicht beachtet.

Erneut wurden aus der Kieferorthopädie die ersten Versuche gestaltet, um ein breiteres Spektrum zu identifizieren. Diese Messungen sollten Aufzeichnungen im negativen sowie im positiven Bereich erlauben, um den Druck zu messen, der von den Weichteilen auf die Zahnreihe ausgeübt wird. Thüer et al. nutzten zu diesen Zwecken im Jahre 1985 ein Mundstück, das über eine Kanüle mit einem Wasserbehälter und komprimierter Luft verbunden war (Thüer et al. 1985). Dieses Messverfahren funktionierte als ein fluidmechanisches System, wobei Druckschwankungen an der Kanüle Druckänderungen im System bewirkten. Diese Druckänderungen wurden von einem Manometer registriert und aufgezeichnet. Mit dieser Methodik konnten Thüer et al. sowie 1991 und 1992 Fröhlich et al. an verschiedenen Oberflächen repräsentativer Zähne im Oberkiefer positive und negative Druckimpulse registrieren (Thüer et al. 1985; Fröhlich et al. 1991; Fröhlich et al. 1992). 
Dieses Messungsspektrum zu erfassen, gelang auch in der bereits erwähnten Studie von Shaker et al. (1988). Rund zwanzig Jahre später wurden Druckänderungen beim Schlucken im positiven und negativen Bereich auch von den Arbeitsgruppen von Kieser (2008) und Kennedy (2010) registriert. Sie verwendeten hierfür MiniaturdruckAufnahmegeräte, die an einer Oberkieferschiene im Gaumenbereich und am Zahnbogen befestigt waren (Kieser et al. 2008; Kennedy et al. 2010). 


\subsection{Ziel der eigenen Untersuchung}

Mittels Manometrie soll die orale Phase des Schluckaktes bei einem gesunden Probandenkollektiv erforscht werden. Die ermittelten Grundlagen sollen als Normwerte für die spätere Interpretation eines klinischen Funktionstests dienen. Die vorgesehen Schluckbedingungen beinhalten Änderungen in der Gabe des Bolus (aktiv und passiv) sowie Konsistenzänderungen des Bolus (Wasser und Gel).

Neben der Messung der Amplituden und der Dauer des entstehenden Druckes sollen auch morphologische Beschreibungen der erhobenen Druckprofile erfasst werden.

Die zu bearbeitenden Fragen während der vorliegenden Arbeit lauten:

1. Werden während der oralen Phase des Schluckaktes intraorale Druckveränderungen der Kompartimente beobachtet?

2. Können diese Druckänderungen dem Schluckakt zugeordnet werden?

3. Sind diese Druckänderungen abhängig von der Art der Bolus-Gabe?

4. Sind diese Druckänderungen abhängig von der Konsistenz des Bolus?

5. Bestehen unter den verschiedenen Bedingungen typische morphologische Merkmale der Druckprofile?

6. Ist eine klinische Anwendung der beschriebenen Untersuchung bei schluckgestörten Patienten möglich?

Das gewonnene Material soll ermöglichen, die Physiologie des Schluckvorganges näher zu verstehen und zu beurteilen. Die durch eine qualitative und quantitative Analyse der Daten gewonnenen Erkenntnisse sollen als Grundlage für zukünftige Untersuchungen dysphagischer Patienten dienen. 


\section{Material und Methode}

\subsection{Das Probandenkollektiv}

Die biometrischen Daten von 52 Probanden wurden für die vorliegende Untersuchung verwendet. Es handelte sich hierbei um gesunde erwachsene Probanden, die sich nach einer ausgehängten Einladung zur Studienteilnahme gemeldet haben. Das Durchschnittsalter betrug 25.48 Jahre mit einer Standardabweichung von 5.68 Jahren, die Altersspanne reichte von 20 bis zu 45 Jahren. Die Geschlechtsverteilung lag bei 42 Frauen und 10 Männern.

Zur Durchführung dieser Studie wurde ein Antrag an das Ethik-Komitee der Universitätsmedizin Göttingen gestellt. Dieser Antrag (Antragsnummer: 24/3/11) wurde positiv begutachtet.

\subsubsection{Ein- und Ausschlusskriterien}

Für die vorliegende Studie wurden gesunde Probanden einbezogen, die eine freiwillige Kooperation auswiesen. Alle Probanden wurden voruntersucht und nach den Ein- und Ausschlusskriterien ausgewählt. Ausschlusskriterien waren: eine mangelhafte Kooperation, Hindernisse bei der Nasenatmung, das Bestehen von orofazialen Dysfunktionen, ein pathologisches Schluckmuster, Sprechstörungen, unvollständige Bezahnung, abnorme Okklusion und das Nutzen von kieferorthopädischen Geräten. Ausgeschlossen wurden auch Probanden mit einer Vorgeschichte chirurgischer Eingriffe im Gesicht- und Halsbereich, abgesehen von Weisheitszahnextraktionen und Tonsillektomien. 


\subsection{Messinstrumente und Geräte}

Die Druckverhältnisse im Mund während des Schluckvorgangs wurden mit Hilfe eines Mundstückes und eines extraoral platzierten Manometers aufgezeichnet. Verbunden wurden diese Elemente durch ein Schlauchsystem.

\subsubsection{Das Mundstück}

Um dem im Mund herrschenden Druck während der Funktion zu messen, wurde eine stabile Positionierung der Schlauchsysteme benötigt. Hierfür wurde ein zur biofunktionellen Therapie geeignetes Mundstück (Silencos ${ }^{\circledR}$, Bredent, Senden, Deutschland) modifiziert und verwendet (Abbildung 8).

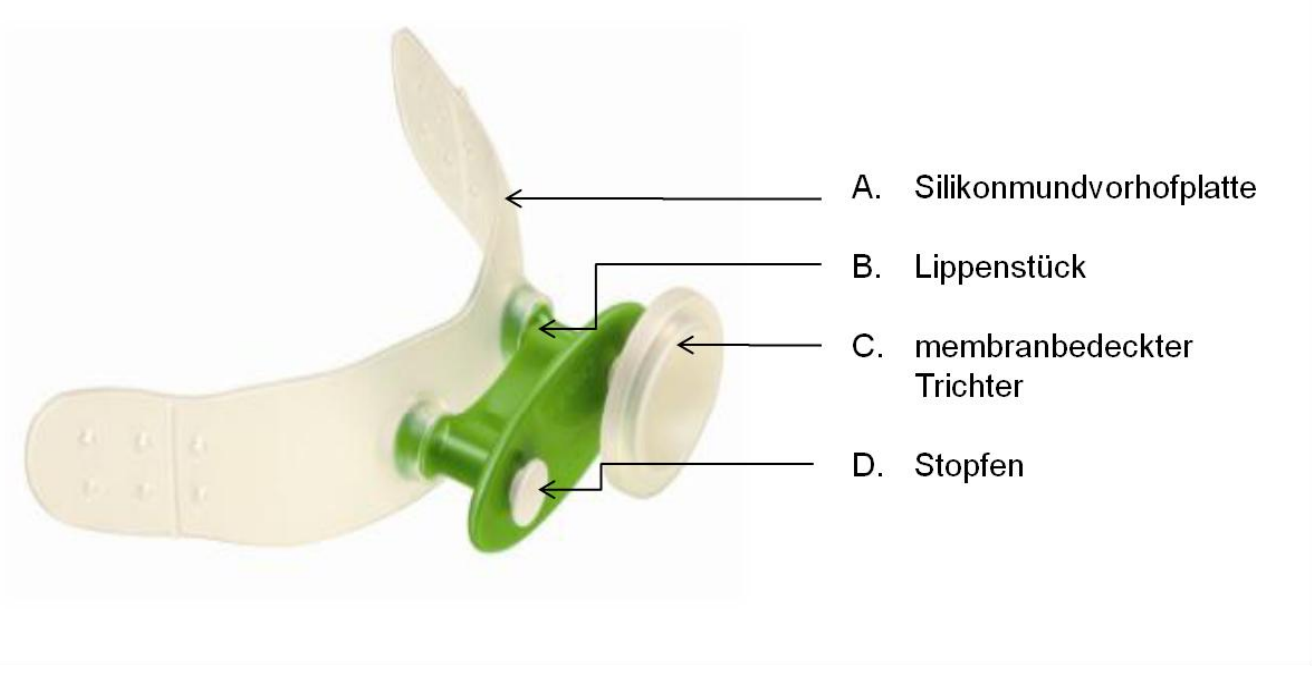

Abbildung 8 Mundstück zur biofunktionellen Therapie der Firma Bredent

Das in der Abbildung 8 dargestellte Mundstück besteht aus einer Mundvorhofplatte aus Silikon und einem Lippenstück. Die Mundvorhofplatte wird vor der Zahnreihe positioniert, gewährt dem Mundstück eine sichere intraorale Stabilität und hält so funktionellen Bewegungen, wie sie zum Beispiel beim Saugen und Schlucken entstehen, stand. Das Lippenstück, das zwischen den Lippen platziert wird, besitzt zwei Eingänge in den intraoralen Raum. Der erste Eingang geht von einem membranbedeckten Trichter aus. Der zweite Eingang ist mit einem Stopfen geschlossen. Die Bedeutung des mit der Membran bedeckten Trichters beruht auf dem Anzeigen der 
intraoralen Druckverhältnisse, da sich die Membran abhängig von den intraoralen Drücken nach außen oder nach innen einziehen kann.

Die unternommene Modifikation des Mundstückes für die Durchführung der Untersuchung ist in der Abbildung 9 dargestellt. Sie basierte auf der Nutzung der Zugänge im Lippenstück für die Zufuhr des Bolus und für die Aufzeichnung von manometrischen Ereignissen. Hierfür wurde ein Silikonschlauch mit einem Außendurchmesser von $3 \mathrm{~mm}$ durch einen der Eingänge geführt. Dieser Schlauch bildete eine Schlaufe, die bis zur Höhe der ersten oberen Molaren reichte und an der zweiten Öffnung des Lippenstücks heraus geführt wurde. Mit einer 2-mm-Stanze wurde die Schlaufe an zwei Punkten perforiert, so dass die Boluszufuhr und die Druckmessung ermöglicht wurden.

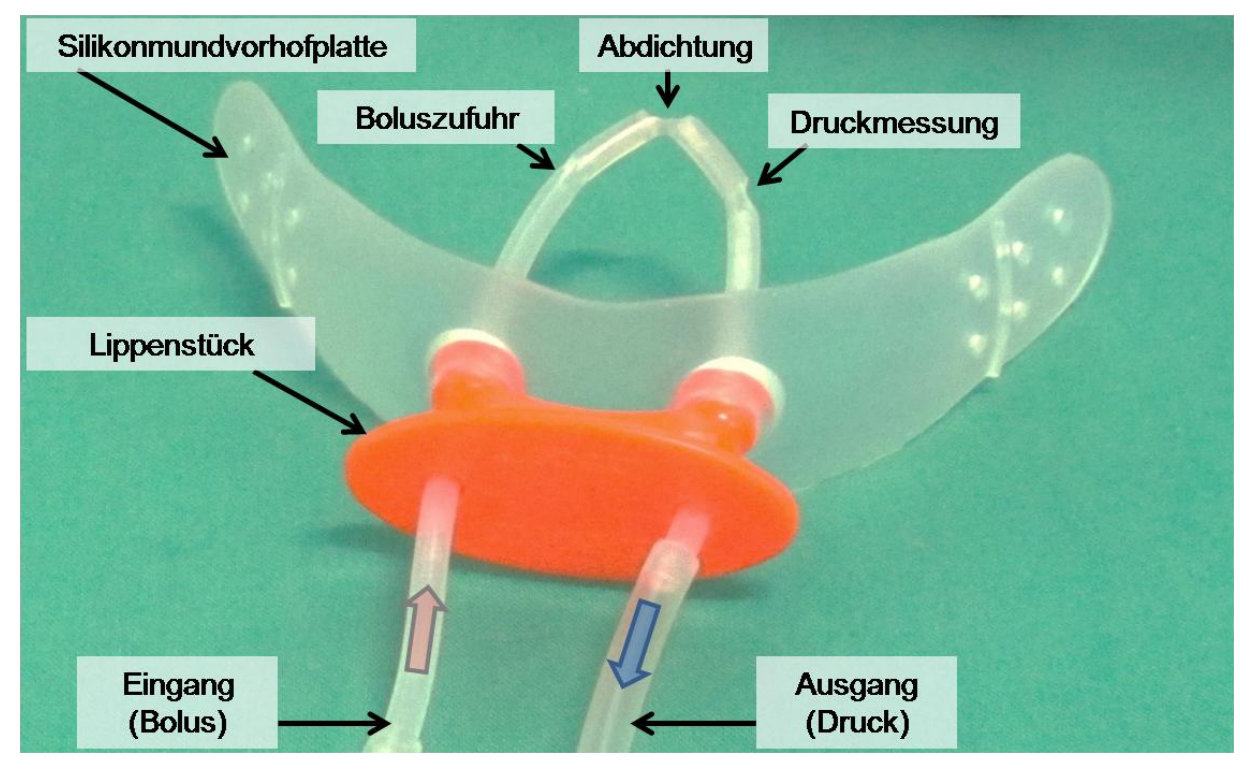

Abbildung 9 Modifiziertes Mundstück mit einer Silikonschlaufe zur Boluszufuhr und intraoralen Druckmessung

Diese beiden Perforationen erlaubten den direkten Zugang zum biofunktionellen Kompartiment 2. Auf diese Art konnte der Messbereich präzise definiert werden. Um eine Abtrennung zwischen den beiden Perforationen (Boluszufuhr und Druckmessung) zu erreichen, wurde am Scheitelpunkt der Schlaufe das interne Lumen mit Kunststoff (Relino Soft ${ }^{\circledR}$, Kanidenta, Herford, Deutschland) gefüllt und auf diese Art verschlossen. Auch das Eindringen des Bolus in den Messbereich wurde mit diesem methodischen Aufbau reduziert. 


\subsubsection{Die Schlauchsysteme}

Außerhalb der Mundhöhle wurden die zwei Zugänge des Mundstückes (Boluszufuhr und Druckmessung) an 4-mm-PVC-Schläuche (Fresenius Kabi ${ }^{\circledR}$, Bad Homburg, Deutschland) weitergeleitet. Einer dieser Schläuche, in der Abbildung 9 als „Eingang“ bezeichnet, reichte zu einer 20-ml-Spritze (B. Braun ${ }^{\circledR}$, Melsungen, Deutschland). Diese Spritze beinhaltete je nach Test verschiedene Flüssigkeiten. Die zweite Verbindung, in der Abbildung 9 ,Ausgang“ benannt, führte über den oben beschriebenen PVCSchlauch zu einem Wasserabscheider (Aqua-Knot II Water Trap®, GE Medical Systems, Milwaukee, WI, USA) und einem Bakterienfilter (Dräger Medical®, Lübeck, Deutschland).

Da während des Schluckvorganges und trotz der Abdichtung der Schlaufe Flüssigkeiten in die Druckmessungsleitung eindringen konnten, diente der Wasserabscheider zum Vermeiden von Messungsfehlern. Zum Schutz der Probanden wurde in der Druckleitung ein Bakterienfilter interponiert. Nach dem Bakterienfilter und über eine medizinische Druckleitung (Anesthesia Gas Sample Line ${ }^{\circledR}$, GE Healthcare, Helsinki, Finnland) wurde der Drucksensor erreicht.

\subsubsection{Das Druckmessgerät}

Das Druckmessgerät bestand aus einem piezoresistiven Sensor (GMSD2BR ${ }^{\circ}$, Greisinger electronics, Regenstauf, Deutschland), der an ein computergesteuertes digitales Manometer (GDUSB 1000®, Greisinger electronics, Regenstauf, Deutschland) angeschlossen wurde. Dieses Druckmessgerät erlaubte Messungen in einem Bereich von 2000 bis -1000 mbar, in einer Messungsfrequenz von $1 \mathrm{kHz}$ und einer Auflösung von 1 mbar. Gesteuert wurde das Manometer mit einem batteriebetriebenen Laptop (Compaq Presario CQ50®, Hewlett-Packard, Palo Alto, CA, USA).

Die Aufhebung der Daten und spätere Bearbeitung der Datensätze gelang mit Hilfe der für Windows geeigneten Software (GSOFT-USB ${ }^{\circledR}$, Greisinger electronics, Regenstauf Deutschland). Eine schematische Darstellung der Versuchsanordnung ist in vereinfachter Form in der Abbildung 10 zu sehen. 


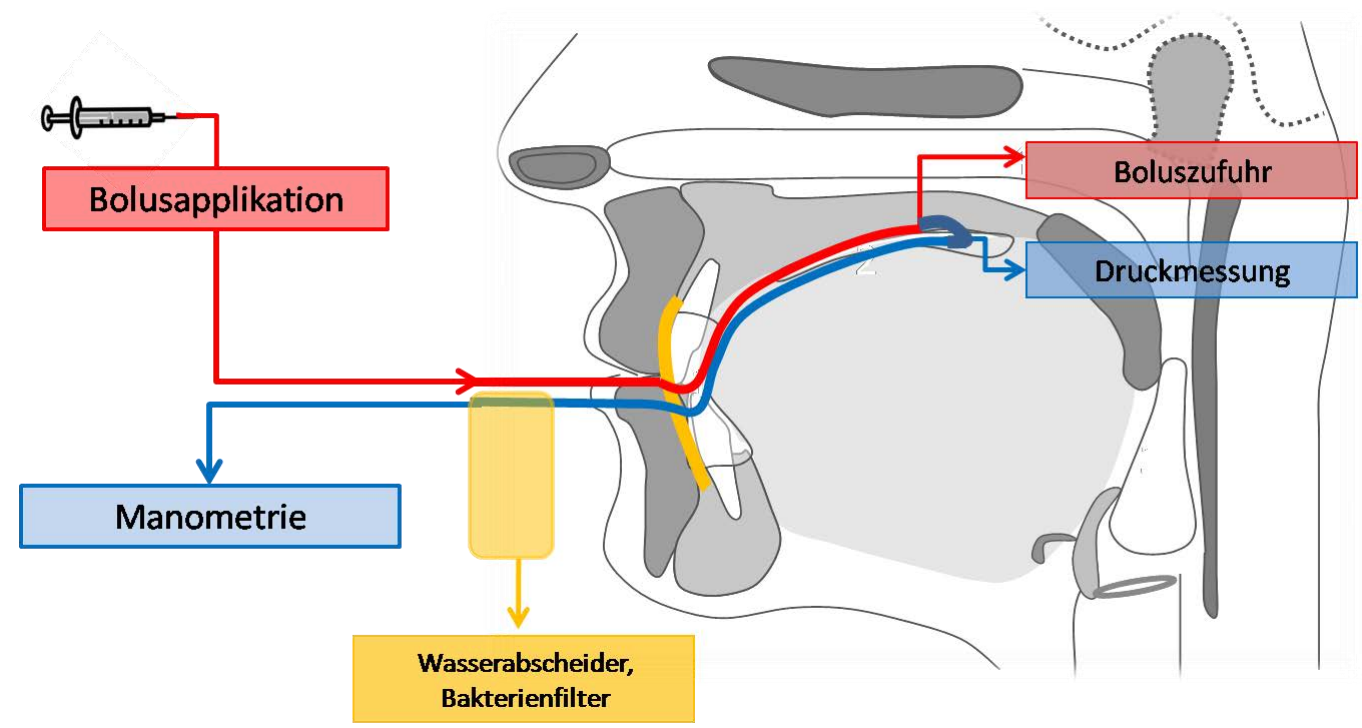

Abbildung 10 Schematische Darstellung der Versuchsanordnung

In diesem schematischen sagittalen Schnitt des orofazialen Systems wird die Lage des intraoralen Mundstückes sowie die extraorale Ableitung über Schlauchsysteme gezeigt. Rot dargestellt ist die Applikation der Flüssigkeit in den Mund (beziehungsweise in Kompartiment 2). Die Spritze stellt das Reservoir für die benutzten Flüssigkeiten dar. Mit Blau ist die Druckableitung aus dem Kompartiment 2 gekennzeichnet. Der Wasserabscheider und der Bakterienfilter vor der Ableitung zur Manometrie werden mit Gelb angezeigt.

Eine grafische Darstellung des gesamten Messaufbaus ist in der Abbildung 11 gegeben. 


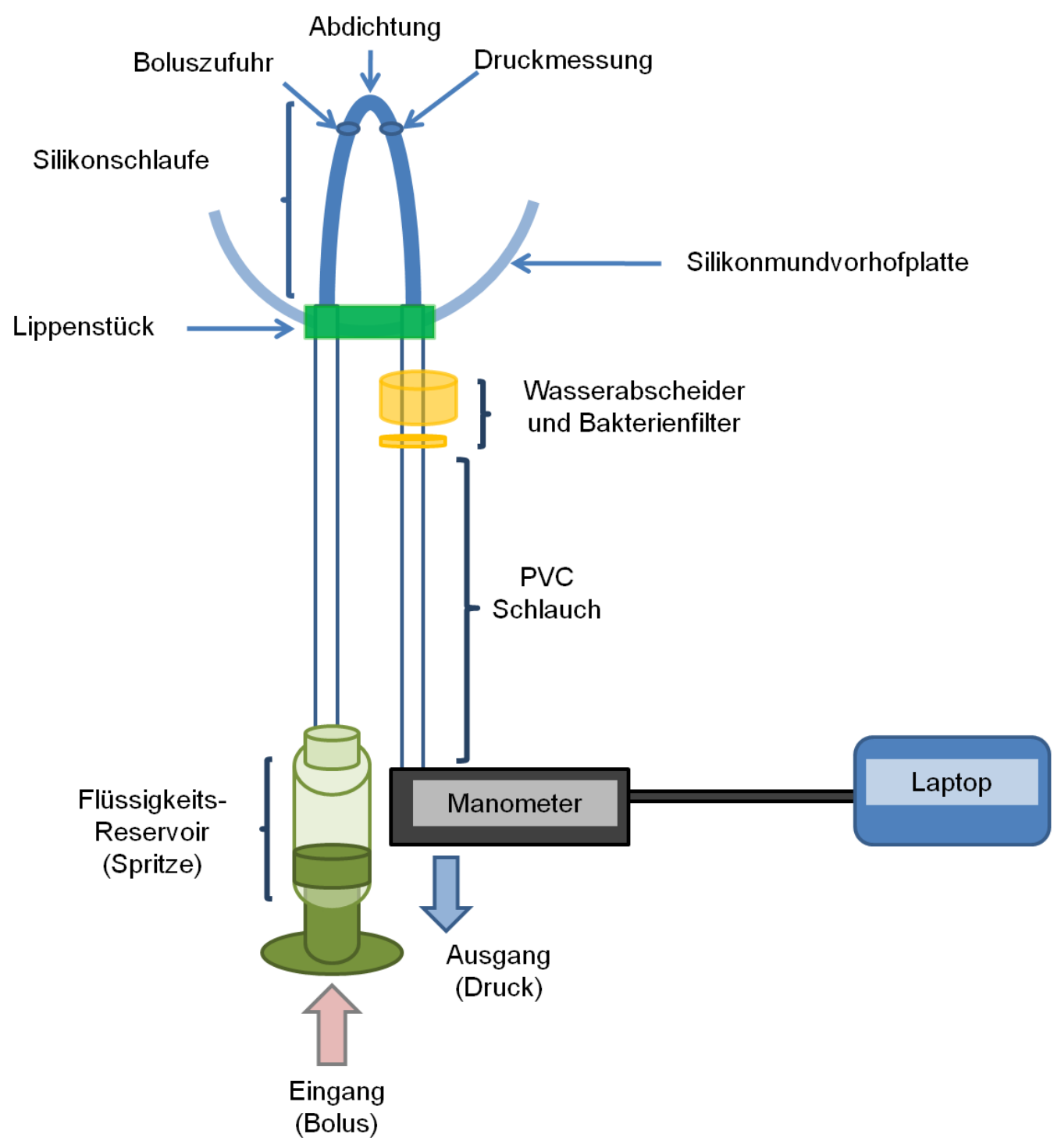

Abbildung 11 Schematische Darstellung des methodischen Aufbaus

\subsubsection{Die verwendeten Flüssigkeiten}

Gemäß der Zielsetzung dieser Studie wurden zwei Bolusarten verwendet, die eine unterschiedliche Konsistenz aufwiesen. Bei den ersten zwei Schluckbedingungen wurden Druckverhältnisse beim Schlucken von Wasser registriert. Hierbei wurde stilles Mineralwasser genutzt (Heil- und Mineralquellen Germeta ${ }^{\circledR}$, Warburg, Deutschland). Bei der dritten Versuchsanordnung wurde angedicktes Wasser (Nutilis Aqua ${ }^{\circledR}$, Nutricia Nutilis, Erlangen, Deutschland) appliziert. Hierbei handelt es sich um ein konsistenzadaptiertes Getränk, welches als medizinisches Produkt zur Nahrungsunterstützung bei dysphagischen Patienten benutzt wird. Dieser gelartige 
Bolus (Abbildung 12) hatte einen angenehmen Orangengeschmack und war amylaseresistent, so dass sich bei dem Kontakt mit Speichel seine Konsistenz nicht veränderte.

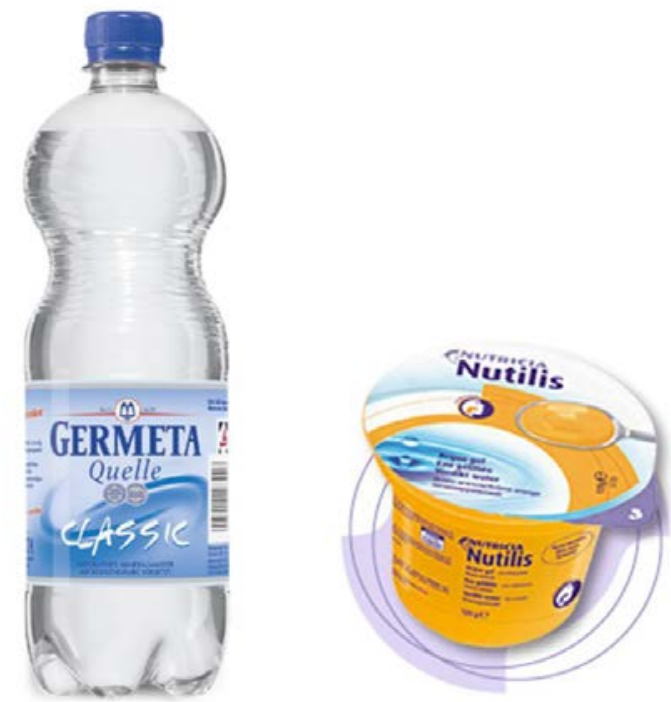

Abbildung 12 Natürliches Mineralwasser und angedicktes Wasser 


\subsection{Durchführung der klinischen Untersuchung}

\subsubsection{Ort der Untersuchung}

Die gesamte Datenerhebung bei den Probanden wurde in Untersuchungseinheiten der Abteilung für Phoniatrie und Pädaudiologie im Zentrum für Augenheilkunde und Hals-, Nasen-, Ohrenheilkunde der Universitätsmedizin Göttingen durchgeführt. Alle Messungen erfolgten unter Betreuung und Aufsicht eines HNO-Facharztes. Die erhobenen Daten wurden bearbeitet im Labor für Polysensografie der Abteilung Mund-, Kiefer- und Gesichtschirurgie der Universitätsmedizin Göttingen.

\subsubsection{Aufklärung der Probanden}

Die Probanden wurden im Vorfeld der Untersuchung über die Messungsanordnung unterrichtet. Schriftlich wurden die Probanden durch ein Informationsblatt über das Ziel und die Art der Versuche sowie über mögliche Risiken aufgeklärt. Nach der Aufklärung wurde von jedem Probanden eine unterschriebene Einverständniserklärung aufbewahrt.

\subsubsection{Anamnese}

Nach der Einverständniserklärung wurden die Probanden nach den bereits erläuterten Ein- und Ausschlusskriterien von einem HNO-Facharzt und einem Zahnarzt untersucht. Die bei dieser Studie gewonnenen Daten und Aussagen der Probanden unterliegen der ärztlichen Schweigepflicht. Die Informationen wurden entsprechend dem Datenschutzrecht behandelt und dienten ausschließlich medizinisch-wissenschaftlichen Aussagen.

\subsubsection{Vorbereitungen der Probanden und Eichung der Geräte}

Für die Untersuchung saßen die Probanden in aufrechter und entspannter Haltung auf einem Stuhl. Vor der intraoralen Messung bekamen die Probanden das Mundstück im Mund platziert, um sie an das Tragen zu gewöhnen. Alle Probanden bekamen vor Untersuchungsbeginn beide verwendeten Flüssigkeiten zum Probieren appliziert. Vorübungen zum Saugen und Schlucken mit dem Mundstück wurden von jedem 
Proband verlangt. Die aus dem Mundstück hervorgehenden Leitungen wurden einerseits mit einer mit Flüssigkeit gefüllten Spritze verbunden und andererseits mit einem Drucksensor (siehe Abbildung 9). Diese beiden Gegenstände wurden vor dem Proband auf dessen Mundhöhe platziert. Auf diese Weise wurden Messungsfehler bezüglich Druckdifferenzen und möglichen Wassersäulen vermieden. Vor jedem Versuch wurden die Geräte geprüft und geeicht. Vor Einsetzen des Mundstückes in den Mund wurde der Druck im Leitungssystem überprüft, der bei 0 mbar (atmosphärischer Druck) liegen sollte. Der Wasserabscheider wurde vor jedem Schlucktest entleert.

\subsubsection{Schluckuntersuchungen}

Mit den oben beschriebenen Bedingungen wurden bei jedem Proband 3 Schluckuntersuchungen durchgeführt. Jede Schluckuntersuchung beinhaltete jeweils 10 Schluckversuche, die mittels einer Mundöffnung getrennt wurden. Während der gesamten Untersuchung wurden manometrische Daten erfasst.

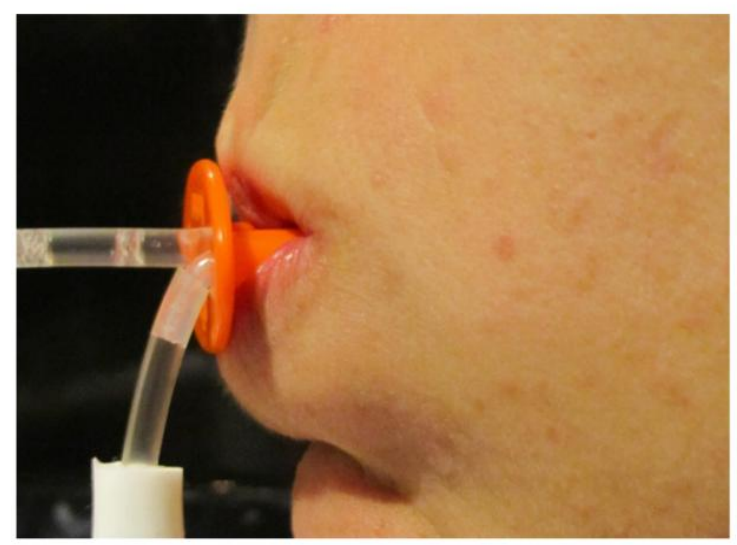

A. Schluckvorgang

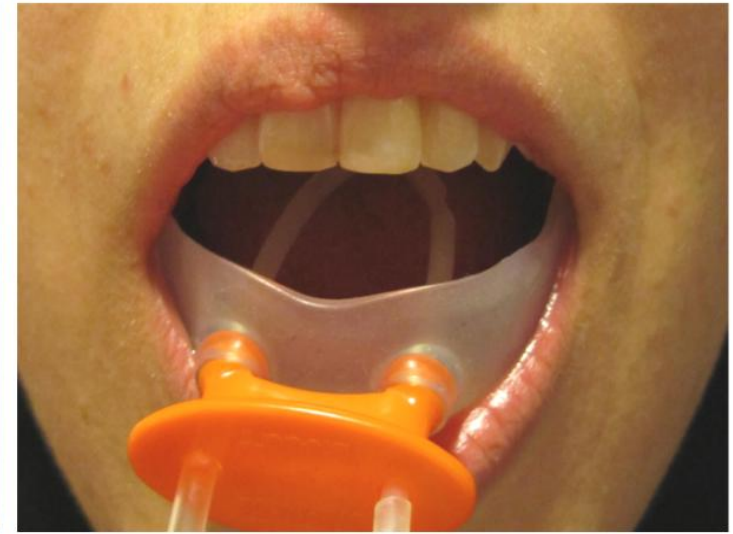

B. Mundöffnung

Abbildung 13 Mundstück in situ während des Tests. Abbildung A: geschlossener Mund während der Bolus-Einnahme. Abbildung B: Darstellung der Mundöffnung zwischen den Schluckvorgängen

\subsubsection{Aktive Bolus-Einnahme (ABE)}

In diesem Versuch wurden die Probanden gebeten, aktiv das Wasser aus der Spritze zu saugen. Um die spontane Saugaktivität zu erfassen, bekamen die Probanden die Anweisung, eine notwendige Menge an Wasser in den Mund aufzunehmen, bis der 
Schluckakt hervorgerufen werden konnte. Anschließend wurden die Versuchspersonen gebeten, $\mathrm{zu}$ schlucken und danach eine Mundöffnung durchzuführen. Diese drei Ereignisse wurden vom Untersucher rhythmisch erläutert, indem folgende Instruktionen laut angesagt wurden: „saugen“ - „schlucken“ - „Mund öffnen“. Die Mundöffnung führte zu einer Unterbrechung der Druckkurve durch atmosphärischen Druck (siehe Abbildung 13B und Abbildung 17).

\subsubsection{Passive Wasser-Bolus-Gabe (PWG)}

Bei diesem Test erfolgte die Gabe eines 2-ml-Wasser-Bolus. Dieses Volumen wurde mit der Spritze injiziert und über das beschriebene Schlauchsystem direkt in Kompartiment 2 appliziert. Die Probanden bekamen von dem Untersucher folgende Ansagen: „Wasser kommt“ - „schlucken“ - Mund öffnen“. Diese Anweisungen erfolgten in einem ruhigen Tempo, so dass die Probanden entspannt und ohne Anstrengung den Test durchführen konnten. Die vorgesehene abschließende Mundöffnung diente zur manometrischen Trennung der Schluckereignisse.

\subsubsection{Passive Gel-Bolus-Gabe (PGG)}

In dieser Versuchsanordnung bekamen die Probanden einen 2-ml-Gel-Bolus (Nutilis Aqua ${ }^{\circledR}$, Nutricia Nutilis, Erlangen, Deutschland) in Kompartiment 2 appliziert. Die Bolus-Gabe erfolgte dabei in gleicher Weise wie bei dem zuvor erläuterten Versuch. Die Anweisungen „Gel kommt“ - „schlucken“ - Mund öffnen“ wurden auch hier in einem ruhigen Rhythmus gegeben. Die abschließende Mundöffnung diente ebenfalls der Abtrennung der manometrischen Schluckereignisse. 


\subsection{Analyse der erhobenen Daten}

Die bei der Untersuchung entstandenen Daten wurden während der Messung in einem batteriebetriebenen Laptop gespeichert. Diese Daten wurden anschließend aus dem Laptop exportiert, auf eine Festplatte transportiert und in einem Computer des Labors für Polysensografie in der Abteilung für Mund-, Kiefer- und Gesichtschirurgie gespeichert und ausgewertet. Aus dem Rohdatensatz wurden alle Daten mit der bereits genannten Software bearbeitet. Für jeden Probanden wurden drei Grafen und tabellarische Datensätze hergestellt, die jeweils den drei untersuchten Schluckbedingungen entsprachen. Eine Abbildung des angewendeten Programms für die Bearbeitung der Daten ist in der Abbildung 14 dargestellt.

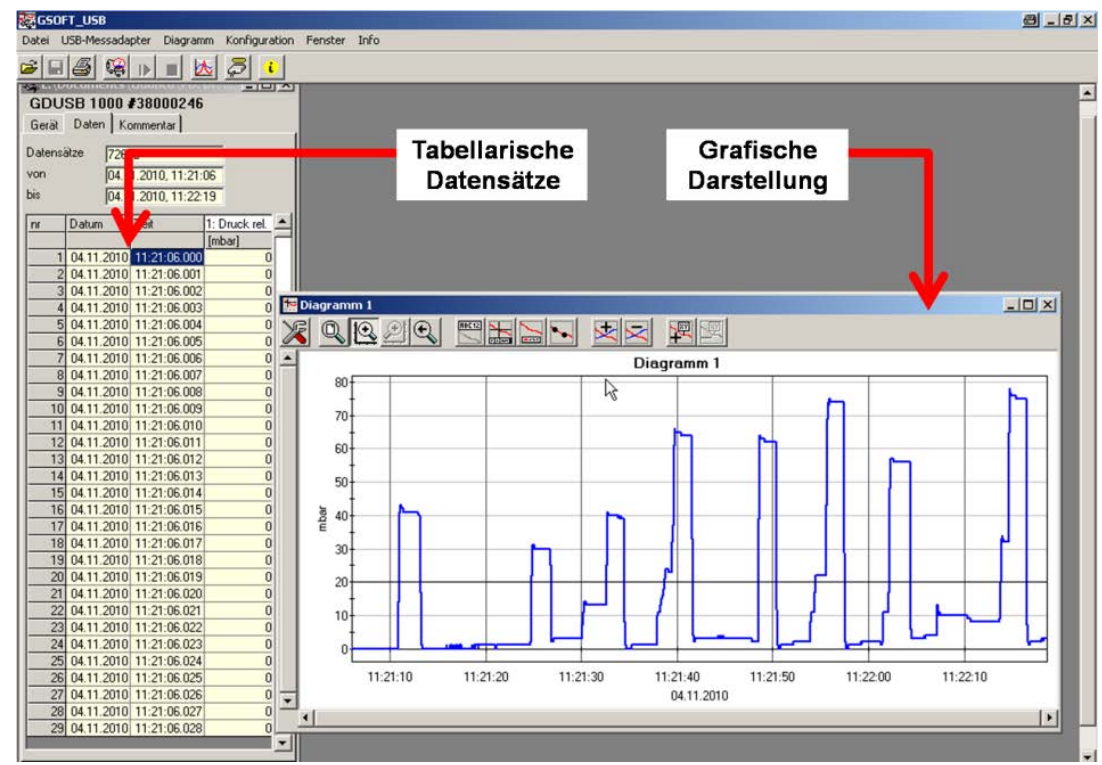

Abbildung 14 Software und Datenpräsentation des Programms GSOFT-USB ${ }^{\circledR}$ 


\subsection{Statistische Methoden}

Bei der Analyse der erhobenen Daten wurden qualitative und quantitative Parameter mit den Programmen Statistica Version 10 (StatSoft GmbH, Hamburg, Deutschland) und SAS Version 9.3 (SAS Institute Inc. Cary, USA) ausgewertet. Das intraindividuelle Verhalten wurde mit der Streuung und den Mittelwerten von jedem einzelnen Probanden dargestellt. Die Beurteilung des gesamten Kollektivs wurde anhand der erfassten Mittelwerte durchgeführt.

Bei allen angewendeten Tests wurde das Signifikanzniveau für die Ablehnung der Nullhypothese auf 5\% festgelegt. Für die Paarvergleiche wurde der p-Wert mittels Bonferroni adjustiert. Folgende Tests wurden angewendet: ANOVA (analysis of variance) für den Globaltest auf Unterschiede zwischen mehreren Gruppen, der T-Test für Paarvergleiche und der McNemar Test für binäre/dichotome Merkmale bei abhängigen Beobachtungen. 


\section{Ergebnisse}

\subsection{Allgemeine Beschreibung der Druckverläufe beim Schluckvorgang.}

Eine Zuordnung von manometrischem Ereignis und Schluckvorgang ließ sich regelmäßig durch negative Druckamplituden bei allen Applikationsarten und Bolusformen erkennen. So konnte für jeden Schluckvorgang eine Druckveränderung erkannt werden, die bezüglich ihrer Morphologie im Sinne der Amplitude und Dauer ausgewertet werden konnte. Der Druck wurde in Millibar (mbar) gemessen, die Zeit in Sekunden (s) (siehe Abbildung 15).

Im Hinblick auf mögliche Messfehler ist die Bildung einer Wassersäule im Schlauchsystem zu berücksichtigen. Diese konnte zwischen dem Mundstück und dem Wasserabscheider auftreten. Die Distanz zwischen dem Messort im subpalatinalen Raum und den Wasserabscheider betrug $5 \mathrm{~cm}$ (siehe Material und Methoden). Daraus resultiert ein möglicher systematischer absoluter Messfehler von 5 mbar bei Annahme einer unbeabsichtigten Wassersäule von $5 \mathrm{~cm}$ im Messsystem. Die Druckamplituden, die im Bereich des Messfehlers beobachtet worden konnten ( -5 mbar bis zu 5 mbar), wurden nicht in die Auswertung der Arbeit miteinbezogen. Nicht nur bei der Amplitude, sondern auch bei der Berechnung der Dauer der einzelnen Druckkurven wurde diese Messfehlergrenze berücksichtigt, indem die Dauer der Ereignisse ab dem Punkt bestimmt wurde, an dem die Kurve einen Wert von 5 mbar erreichte und auf diesen zurückfiel (Abbildung 15).

In der vorliegenden Arbeit ist $\mathrm{zu}$ beachten, dass der Zeitverlauf auf der X-Achse aufgetragen ist; die Amplituden auf der Y-Achse. Negative Druckamplituden werden oberhalb der X-Achse und positive Amplituden unterhalb der X-Achse abgebildet, wie es in der Abbildung 15 schematisch dargestellt wird. 


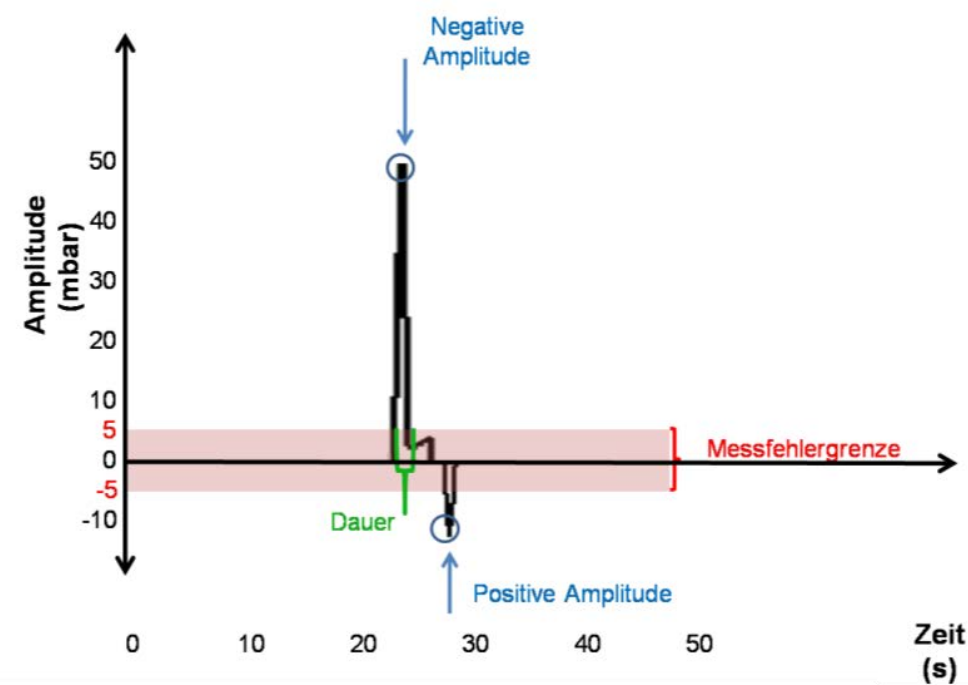

Abbildung 15 Merkmale der manometrischen Messung (Amplitude und Dauer) bei einem Schluckversuch. Darstellung der Messfehlergrenze bei positiven und negativen Druckverläufen

Insgesamt wurden bei jedem Probanden 3 Tests durchgeführt. Die Durchführung der Tests wies keine Probleme auf. Alle Probanden akzeptierten den methodischen Aufbau, konnten die verlangten Aufgaben fehlerfrei ausführen und benötigten keine zusätzlichen Erklärungen. Keiner der Probanden verschluckte sich bei der Untersuchung oder wies den Bolus zurück.

Bei jedem Test wurden 10 Schluckvorgänge aufgezeichnet, die grafisch dargestellt werden konnten. Für die gesamte Studie wurden so 156 Aufnahmen mit insgesamt 1560 Schluckvorgängen erstellt. Da nach jedem Schluckvorgang eine Mundöffnung verlangt wurde, konnten Druckschwankungen, die bei jedem Schluckvorgang erzeugt wurden, durch Druckamplituden mit dem atmosphärischen Druck (0 mbar) unterbrochen werden.

Beispiele für die drei Tests sind in den Abbildungen 16 bis $18 \mathrm{zu}$ sehen. 


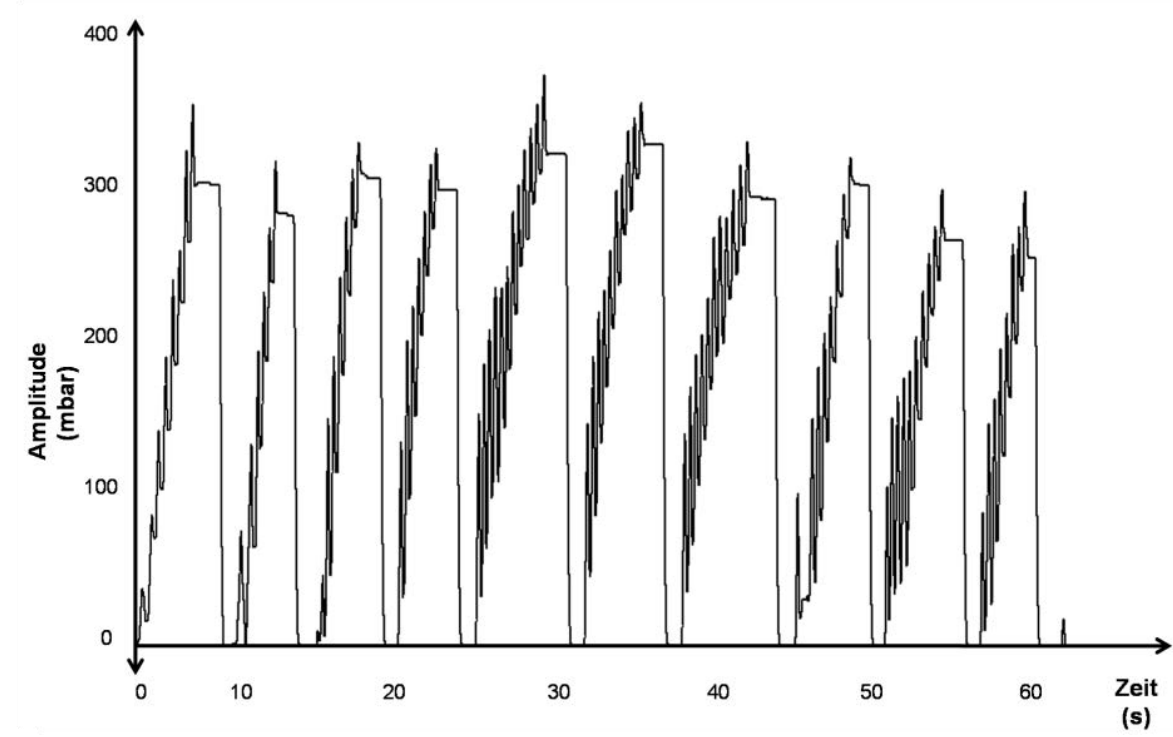

Abbildung 16 Druckdiagramm eines Probanden bei der aktiven Bolus-Einnahme. Die Mundöffnung wird durch einen Druckausgleich (0 mbar) zwischen den BolusEinnahmen gekennzeichnet

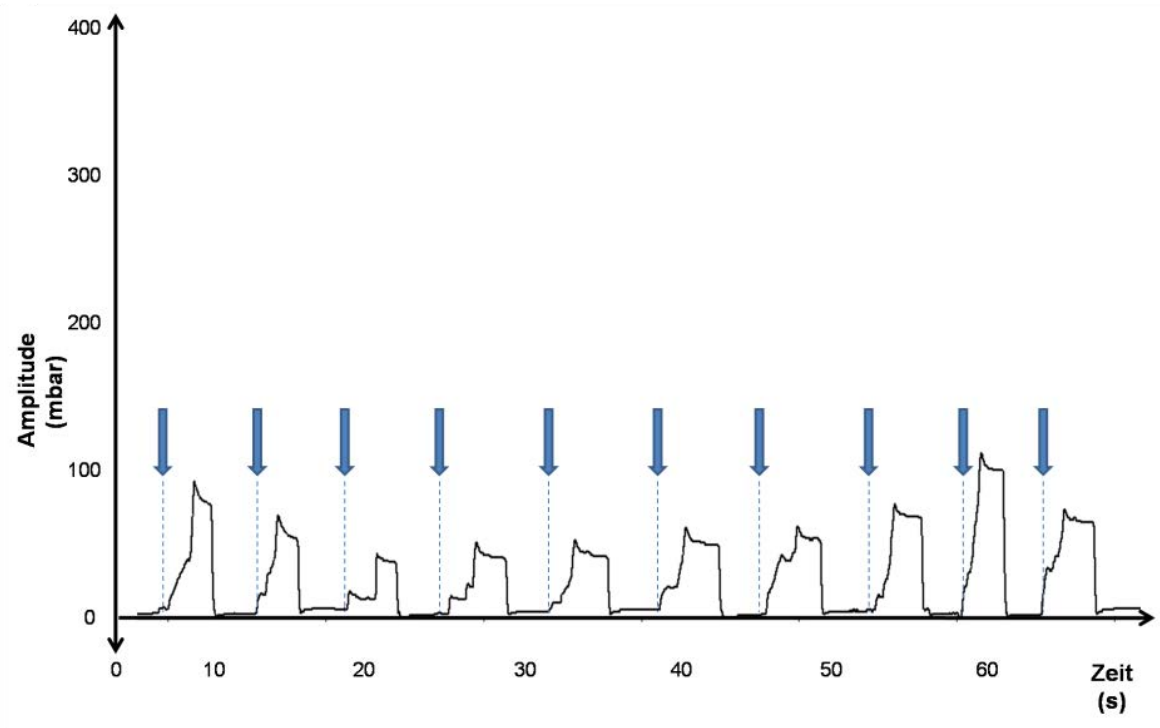

Abbildung 17 Druckdiagramm eines Probanden bei der passiven Wasser-Bolus-Gabe. Mit einem blauen Pfeil wird die Bolusapplikation gekennzeichnet, die Mundöffnung wird durch einen Druckausgleich (0 mbar) zwischen den Amplituden gekennzeichnet 


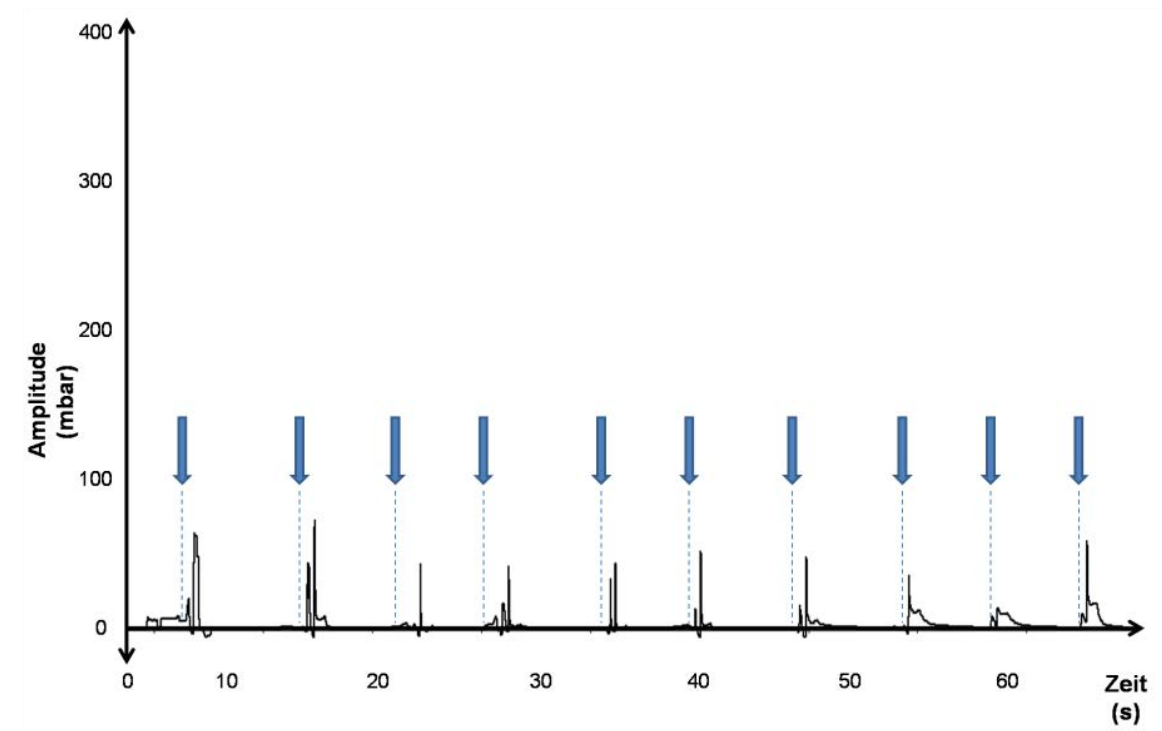

Abbildung 18 Druckdiagramm eines Probanden bei der passiven Gel-Bolus-Gabe. Mit einem blauen Pfeil wird die Bolusapplikation gekennzeichnet, die Mundöffnung wird durch einen Druckausgleich (0 mbar) zwischen den Amplituden gekennzeichnet 


\subsection{Intraindividuelle Variabilität}

Das individuelle Verhalten jedes einzelnen Probanden im Verlauf der Untersuchung konnte anhand von dessen Standardabweichung (SD) dargestellt werden. Für die Auswertung des gesamten Kollektivs wurde hingegen der Mittelwert genutzt. Die Auflistung aller Mittelwerte und SD der einzelnen Probanden ist im Anhang zu finden. Ein Beispiel der ersten fünf untersuchten Probanden ist in der Tabelle 1 dargestellt.

\begin{tabular}{l|c|c|c|c|c}
\hline Proband & Test & $\begin{array}{c}\text { MW } \\
\text { Amplitude }\end{array}$ & $\begin{array}{c}\text { SD } \\
\text { Amplitude }\end{array}$ & $\begin{array}{c}\text { MW } \\
\text { Dauer }\end{array}$ & $\begin{array}{c}\text { SD } \\
\text { Dauer }\end{array}$ \\
\hline \multirow{4}{*}{1} & ABE & 287.3000 & 46.2530 & 5.650300 & 1.196565 \\
\cline { 2 - 6 } & PWG & 31.7000 & 15.6706 & 5.256300 & 3.218566 \\
\cline { 2 - 6 } & PGG & 67.6000 & 50.2819 & 3.239800 & 1.859435 \\
\hline \multirow{4}{*}{3} & ABE & 376.2000 & 52.9545 & 4.525100 & 1.239039 \\
\cline { 2 - 6 } & PWG & 41.6000 & 16.5677 & 2.949000 & 0.824347 \\
\cline { 2 - 6 } & PGG & 85.0000 & 44.0202 & 1.126400 & 0.640064 \\
\hline \multirow{4}{*}{4} & ABE & 274.4000 & 23.5947 & 3.973000 & 0.259466 \\
\cline { 2 - 6 } & PWG & 38.6000 & 15.7353 & 2.396000 & 0.935602 \\
\cline { 2 - 6 } & PGG & 31.3000 & 17.3849 & 2.208200 & 1.056706 \\
\hline & ABE & 182.3000 & 18.1233 & 4.107900 & 0.672037 \\
\cline { 2 - 6 } & PWG & 22.5000 & 16.5613 & 2.430200 & 1.857629 \\
\cline { 2 - 6 } & PGG & 23.0000 & 10.6458 & 5.102800 & 2.622470 \\
\hline 5 & ABE & 123.8000 & 19.7191 & 5.901900 & 1.259777 \\
\cline { 2 - 6 } & PWG & 104.5000 & 22.1973 & 6.018600 & 1.270848 \\
\cline { 2 - 6 } & PGG & 74.5000 & 18.1246 & 2.562600 & 0.948809 \\
\hline
\end{tabular}

Tabelle 1 Beispiel der erhobenen Mittelwerte und Standardabweichungen der Amplituden und Dauer bei der gesamten Untersuchung bei 5 Probanden

Alle gewonnenen Kurven wurden anhand der Amplituden und der Dauer analysiert. Eine grafische Darstellung der Mittelwerte der erhobenen Amplituden und der Dauer im Verlauf der gesamten Untersuchung (ABE, PWG, PGG) ist in den Abbildungen 22 für die Amplitude und in der Abbildung 23 für die Dauer dargestellt. Genauer beschrieben werden diese Werte in den nächsten Abschnitten. 


\subsection{Qualitative Auswertung in Abhängigkeit von der Schluckmodalität}

Ein unterschiedlicher Druckverlauf konnte in Abhängigkeit von den verschiedenen Modalitäten erkannt werden. Diese Muster ließen sich qualitativ beschreiben und erlaubten eine Unterteilung der Probanden.

\subsubsection{Aktive Bolus-Einnahme (ABE)}

Bei der aktiven Einnahme eines Bolus konnten zwei Merkmale der Kurve separat beschrieben werden. Ein einfacher Verlauf war durch eine lineare Steigung des Druckes, ein mehrfacher Aufbau durch wiederholte ansteigende Druckpeaks charakterisiert (Abbildung 19).

Der Druckabfall konnte anhand von zwei Mustern beschrieben werden: Ein stufenförmiger Abfall und ein rascher Druckabfall, bei dem ein abrupter Abfall zurück zur Nulllinie beobachtet werden konnte (Abbildung 19).

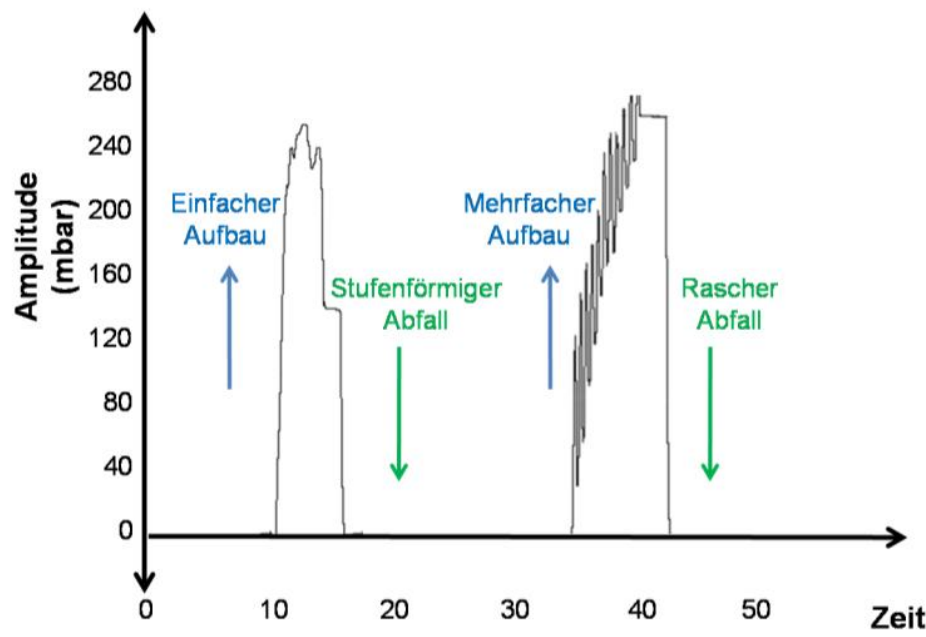

(s)

Abbildung 19 Grafische Darstellung der morphologischen Merkmale bei der aktiven Bolus-Einnahme

\subsubsection{Passive Wasser-Bolus-Gabe (PWG)}

In dieser Modalität konnte das Auftreten von positiven Druckverläufen mit niedrigeren Amplituden als Ergänzung zu den dem Schluckakt zugeordneten Druckschwankungen 
erkannt werden. Dieses morphologische Merkmal wird in der Abbildung 20 gezeigt. Polyphasische Kurven wurden bei der passiven Gabe eines Bolus aus Wasser nicht erkannt.

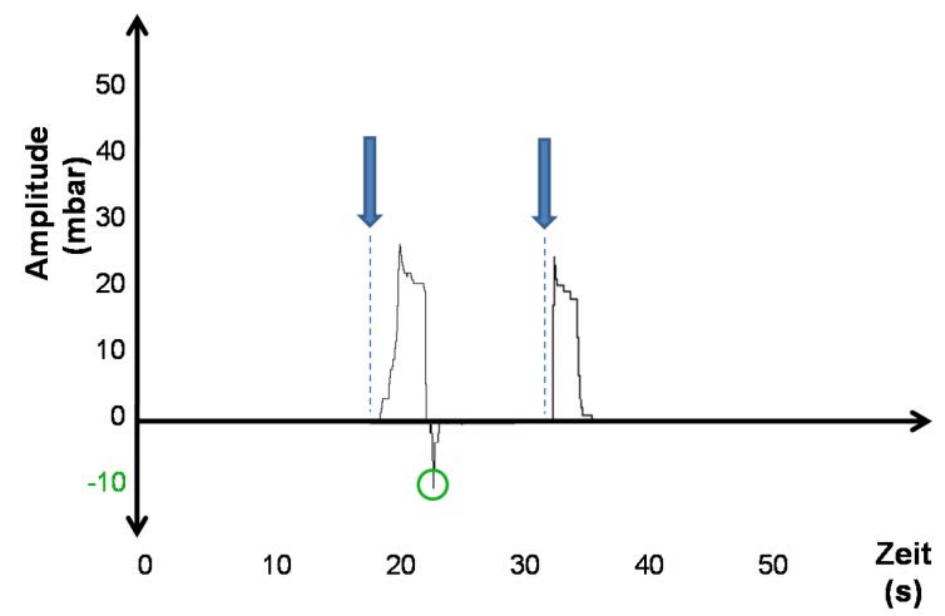

\begin{abstract}
Abbildung 20 Beispiele für eine Druckkurve mit terminal positivem Druck (grüne Markierung) und ohne. Mit einem blauen Pfeil wird der Zeitpunkt der Bolusgabe gekennzeichnet
\end{abstract}

\title{
3.3.3. Passive Gel-Bolus-Gabe (PGG)
}

Bei der passiven Gabe eines Bolus aus Gel konnte erneut das Auftreten von positiven Druckamplituden (Abbildung 20) erkannt werden. Zusätzlich konnte bei der morphologischen Beschreibung des Kurvenverlaufs eine monophasische und eine polyphasische Kurve erkannt werden. Die Definition für eine „monophasische Kurve“ basiert auf einer Druckschwankung mit einem einzigen Aufbau und Druckabfall. Im Gegensatz hierzu wird eine ,polyphasische Kurve“ von einem mehrfachen Umschlag der Druckkurve gekennzeichnet, so dass die durch den Schluckakt entstandene Kurve mindestens zwei Peaks beschreibt. Diese polyphasische Charakterisierung erinnert an einen M-artigen Verlauf. Dargestellt sind beide Kurvenarten in der Abbildung 21. 


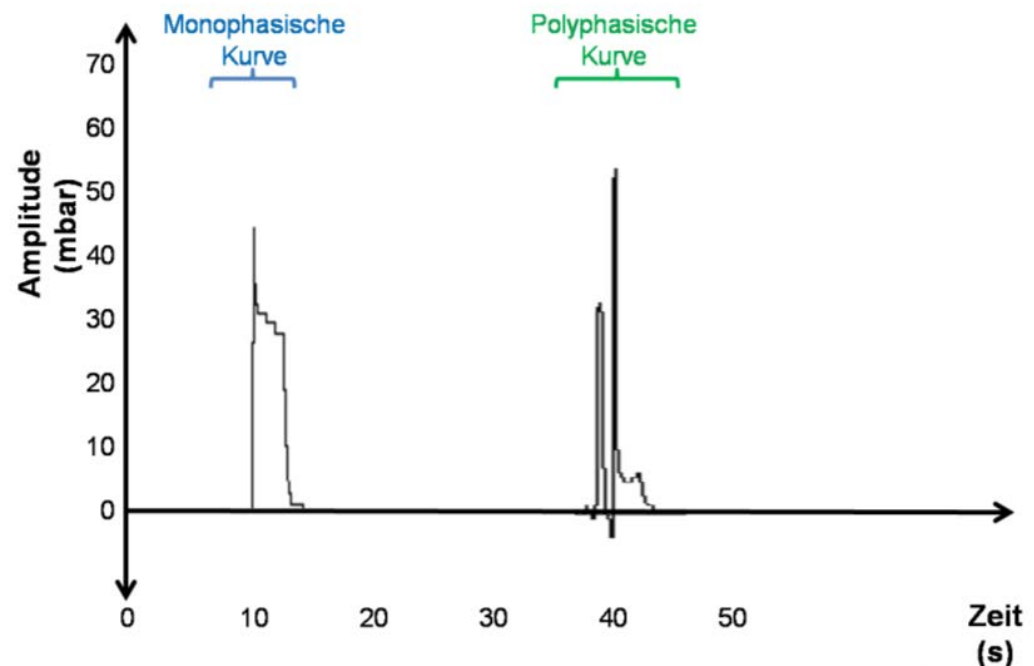

Abbildung 21 Beispiele für eine monophasische und eine polyphasische Kurve bei der passiven Gabe eines Bolus 


\subsection{Quantitative Auswertung in Abhängigkeit von den Modalitäten}

Bei der quantitativen Auswertung der Untersuchung wurden die Amplitude und die Dauer der Vorgänge gemessen. Signifikante Unterschiede zwischen den drei Modalitäten konnten bezüglich der Amplitude $(p<0.0001)$ und der Dauer $(p<0.0001)$ erkannt werden. Eine grafische Darstellung der erhobenen Daten und Vergleiche zwischen den Gruppen werden nachfolgend erwähnt.

\subsection{1. Übersicht über die Messung}

In der Abbildung 22 werden die erhobenen Mittelwerte der Amplituden aller Probanden bei den drei untersuchten Modalitäten dargestellt.

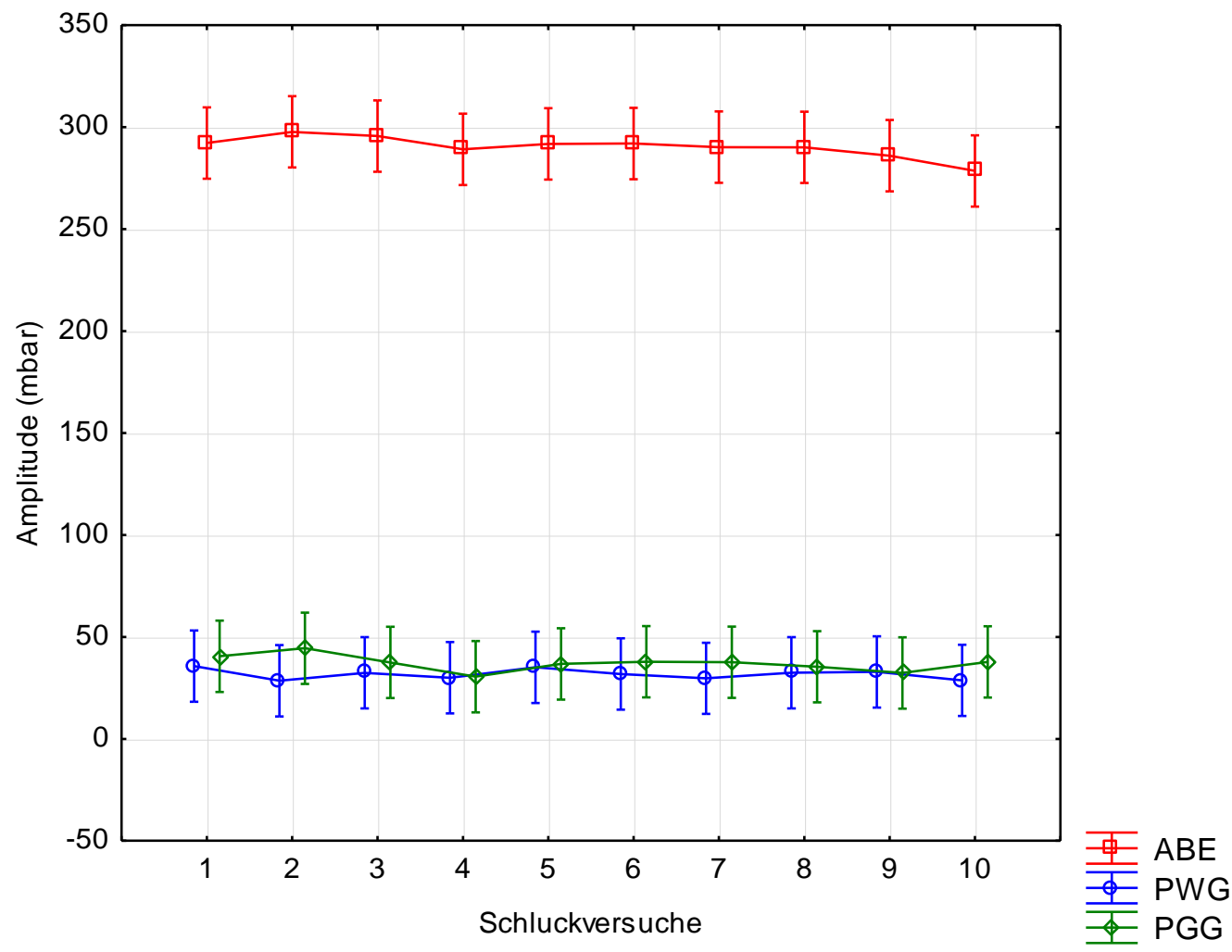

\footnotetext{
Abbildung 22 Grafische Darstellung der Mittelwerte der erhobenen Amplituden bei jedem Schluckvorgang in der gesamten Untersuchung bei allen Probanden. Die vertikalen Linien beschreiben das Konfidenzintervall von 95\%. Jeder Test wird mit einer farbigen Linie dargestellt, in der vorliegenden Grafik steht die rote Linie für die aktive Bolus-Einnahme, die Blaue für die passive Gabe eines Bolus aus Wasser und die Grüne für die passive Gabe eines Bolus aus Gel
} 
In der Abbildung 23 werden die erhobenen Mittelwerte der Dauer von allen Probanden bei den drei untersuchten Modalitäten dargestellt.
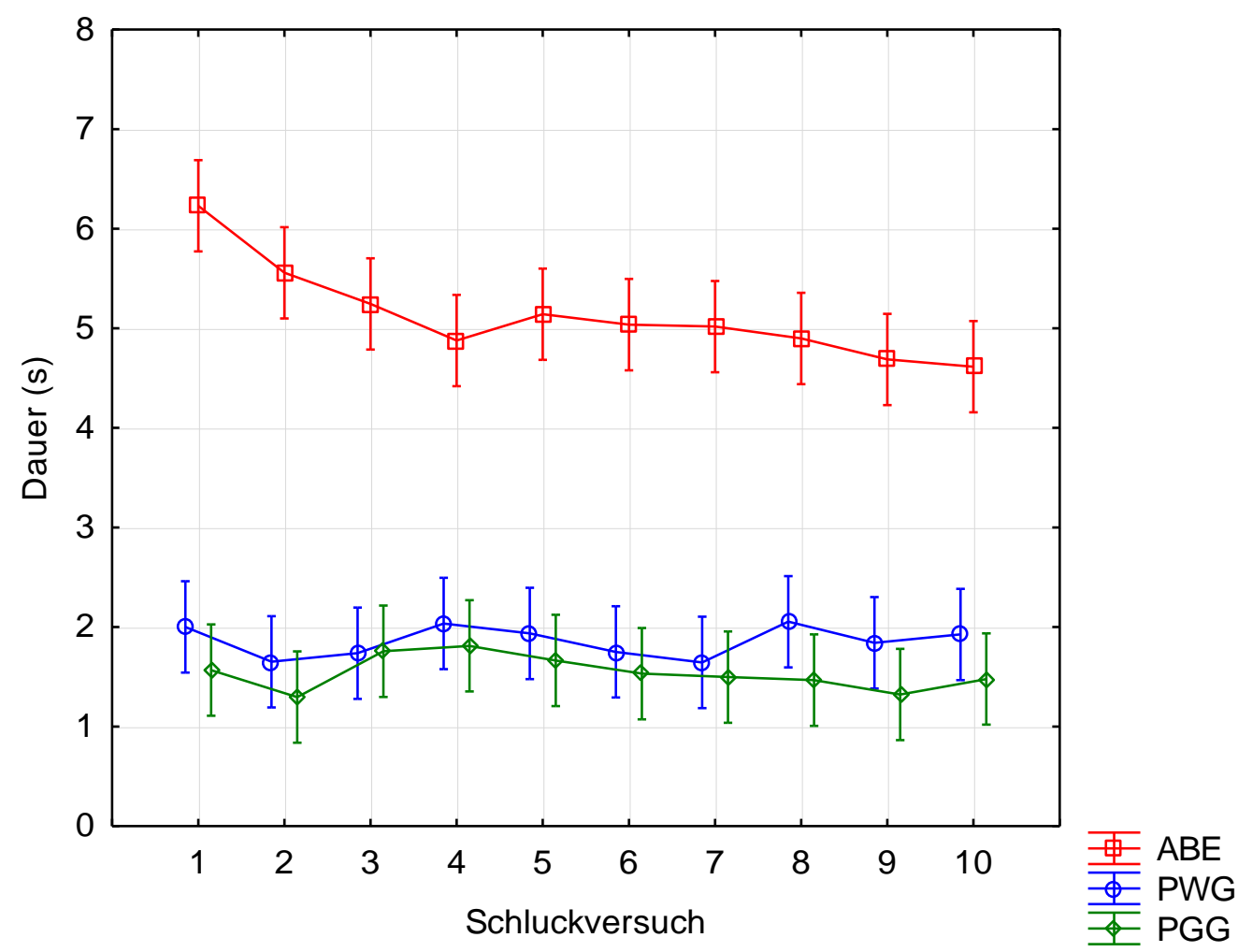

Abbildung 23 Grafische Darstellung der Mittelwerte der erhobenen Dauer bei jedem Schluckvorgang in der gesamten Untersuchung und bei allen Probanden. Die vertikalen Linien beschreiben das Konfidenzintervall von 95\%. Jeder Test wird mit einer farbigen Linie dargestellt, in der vorliegenden Grafik steht die rote Linie für die aktive BolusEinnahme, die Blaue für die passive Gabe eines Bolus aus Wasser und die Grüne für die passive Gabe eines Bolus aus Gel

Anhand der Applikationsart konnten signifikante Unterschiede zwischen den Mittelwerten der Amplitude und der Dauer erkannt werden. Zwischen den beiden passiven Untersuchungen konnte keine signifikante Differenz erkannt werden. Siehe dazu auch Tabelle 2. 


\begin{tabular}{lllcl}
\hline Modalität & Modalität & MW 1 & MW 2 & p-Wert \\
\hline PWG & ABE & 31.7134615 & 290.35 & $<0.0001$ \\
& PGG & 31.71346 & 37.05769 & 0.305122488 \\
ABE & PGG & 290.35 & 37.0576923 & $<0.0001$
\end{tabular}

Tabelle 2 Vergleich (T-Test) zwischen den erhobenen Mittelwerten der Amplituden bei den unterschiedlichen Modalitäten

Bei der Auswertung der Dauer der Schluckereignisse konnte auch ein signifikanter Unterschied zwischen der aktiven Einnahme und den beiden passiven Modalitäten beobachtet werden. Zwischen beiden passiven Modalitäten (Wasser- und Gel-Gabe) konnten keine signifikanten Abweichungen erkannt werden. Siehe dazu auch Tabelle 3.

\begin{tabular}{lllll}
\hline Modalität & Modalität & MW 1 & MW2 & p-Wert \\
\hline PWG & ABE & 1.85727846 & 5.13040288 & $<0.0001$ \\
& PGG & 1.85727846 & 1.53893269 & 0.152425932 \\
ABE & PGG & 5.13040288 & 1.53893269 & $<0.0001$ \\
\hline
\end{tabular}

Tabelle 3 Vergleich (T-Test) zwischen den erhobenen Mittelwerten der Dauer bei den unterschiedlichen Modalitäten

\subsubsection{Aktive Bolus-Einnahme (ABE)}

Bei der aktiven Einnahme eines Bolus wurde bei den Amplituden ein Mittelwert von -290 mbar beobachtet. Die Amplitude hatte ein konstantes Verhalten während der 10 Schluckvorgänge (siehe Abbildung 22). Die Dauer der Druckkurven betrug $5.1 \mathrm{~s}$ und nahm im Verlauf der Untersuchung signifikant ab (siehe Abbildung 23). Der Vergleich zwischen dem Mittelwert der gemessenen Dauer beim ersten und beim zehnten Versuch ergab einen signifikanten Unterschied mit $\mathrm{p}<0.0003$.

Ein relevanter Zusammenhang zwischen Amplitude und Dauer konnte nicht erkannt werden, obwohl die Tendenz zu einer kürzeren Dauer bei geringeren Amplituden in der grafischen Darstellung bestand, was sich aber statistisch anhand des r-Wertes nicht als eine starke Korrelation beweisen ließ $(\mathrm{r}=0.205)$. 


\subsubsection{Passive Wasser-Bolus-Gabe (PWG)}

Bei der passiven Gabe eines Bolus aus Wasser wurde einen Mittelwert von -31 mbar beobachtet. Der Mittelwert der erhobenen Amplitude zeigte während des Verlaufs der 10 Versuche keine Veränderung (siehe Abbildung 22). Der Mittelwert der Dauer der erhobenen Kurven betrug 1.8 s. Im Hinblick auf den zeitlichen Ablauf konnte keine signifikante Änderung der Dauer erkannt werden, die Dauer des ersten und des zehnten Versuches wurden hierfür mit Hilfe des T-Tests verglichen $(p=0.8)$.

Eine Korrelation zwischen Amplitude und Dauer ergab bei der passiven Applikation eines Bolus aus Wasser einen relevanten Zusammenhang. Hierbei konnte bei denjenigen Probanden, die höhere Amplituden aufwiesen, anhand des Korrelationskoeffizienten $(\mathrm{r}=0.6491)$ auch eine längere Dauer der Ereignisse erkannt werden (Abbildung 24).

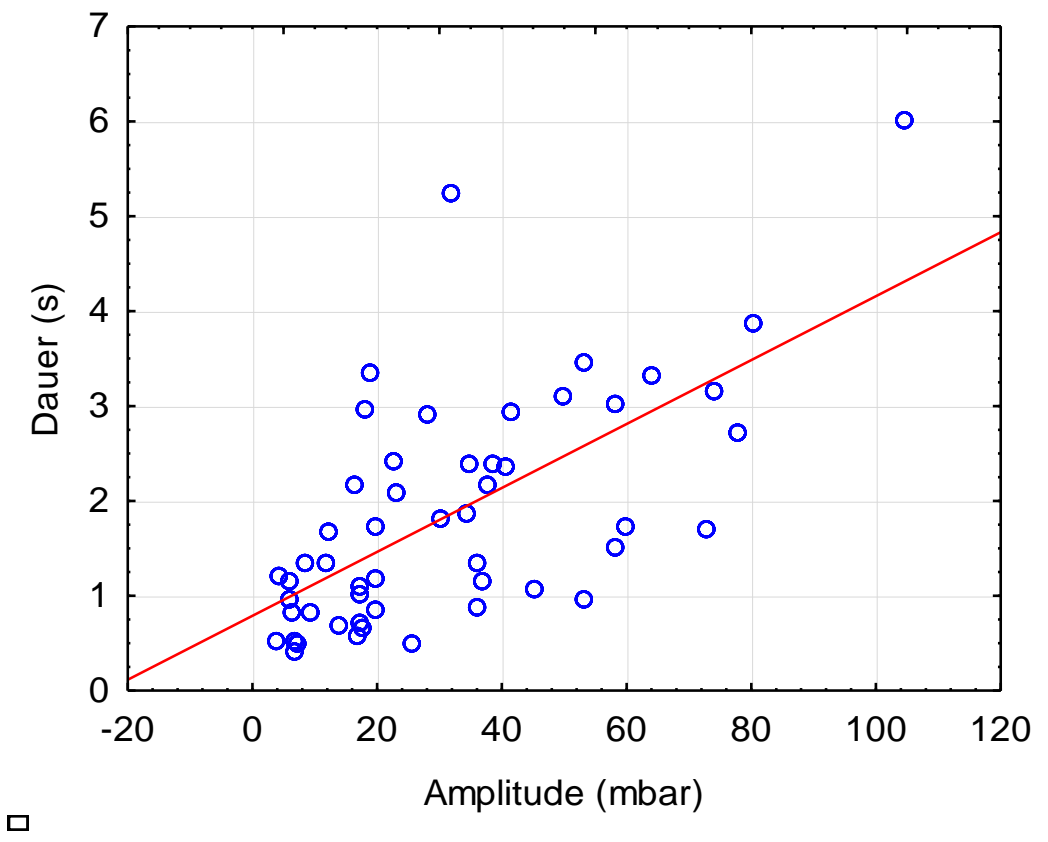

Abbildung 24 Streudiagramm der Mittelwerte der Amplituden und Dauer bei der Applikation eines Bolus aus Wasser

\subsubsection{Passive Gel-Bolus-Gabe (PGG)}

Die Amplituden bei der passiven Applikation eines Bolus aus Gel hatten einen Mittelwert von -37 mbar. Während der Untersuchung konnte keine signifikante zeitliche Tendenz oder Veränderung erkannt werden (siehe Abbildung 22). 
Der Mittelwert der Dauer der Kurven betrug 1.5 s. Im Verlauf der Untersuchung wies die Dauer der Druckkurven keine signifikante Veränderung oder Tendenz auf. Dies ist dem Vergleich zwischen der Dauer des ersten und des zehnten Versuches zu entnehmen $(p=0.58)$.

Auch bei der Korrelation von Amplitude und Dauer konnte trotz der tendenziellen Beobachtung, dass Probanden mit einer höheren Amplitude auch eine längere Dauer auswiesen, kein relevanter Zusammenhang anhand des Korrelationskoeffizienten $(r=0.205)$ erkannt werden. 


\subsection{Spezifische Korrelationen von quantitativen und qualitativen Merkmalen}

Bei der Auswertung der Kurven in Abhängigkeit von den verschiedenen Modalitäten wurden qualitative Parameter, die eine Unterteilung der Probanden erlaubten, festgestellt. Ein Vergleich der entstandenen Gruppen anhand ihrer Amplituden und Dauer konnte anschließend durchgeführt werden.

\subsubsection{Aktive Bolus-Einnahme (ABE)}

Bei der aktiven Einnahme eines Bolus wurde gesondert auf die Kurvenmorphologie im Sinne des beschrieben Druckaufbaus und Druckabbaus - geachtet. Bei diesen zwei morphologischen Merkmalen konnte eine Korrelation mit der Amplitude und Dauer durchgeführt werden.

Bei der Beschreibung des Druckaufbaus konnten zwei Muster erkannt werden: der einfache Aufbau des Druckes, der bei 37 Probanden zu finden war, und ein mehrfacher Aufbau, der bei den restlichen 15 Probanden beobachtet werden konnte. Signifikante Unterschiede konnten zwischen beiden Gruppen erkannt werden. Die Probanden mit einem mehrfachen Aufbau zeigten höhere Amplituden und eine längere Dauer der Vorgänge $(p<0.05)$. Dies wird in der Tabelle 4 zusammengefasst und in den Abbildungen 25 und 26 anhand von Box-Plots dargestellt.

\begin{tabular}{lccccc}
\hline & $\begin{array}{c}\text { MW } \\
\text { Einfach }\end{array}$ & $\begin{array}{c}\text { MW } \\
\text { Mehrfach }\end{array}$ & $\begin{array}{c}\text { SD } \\
\text { Einfach }\end{array}$ & $\begin{array}{c}\text { SD } \\
\text { Mehrfach }\end{array}$ & p-Wert \\
& 262.6405 & 358.7000 & 79.96492 & 87.50833 & 0.001193 \\
Amplitude & 2601093 & 1.590142 & 1.704035 & 0.024839 \\
\hline
\end{tabular}

Tabelle 4 Vergleich (T-Test) zwischen beiden Merkmalen (einfacher und mehrfacher Aufbau) und Amplitude und Dauer bei der aktiven Einnahme eines Bolus 


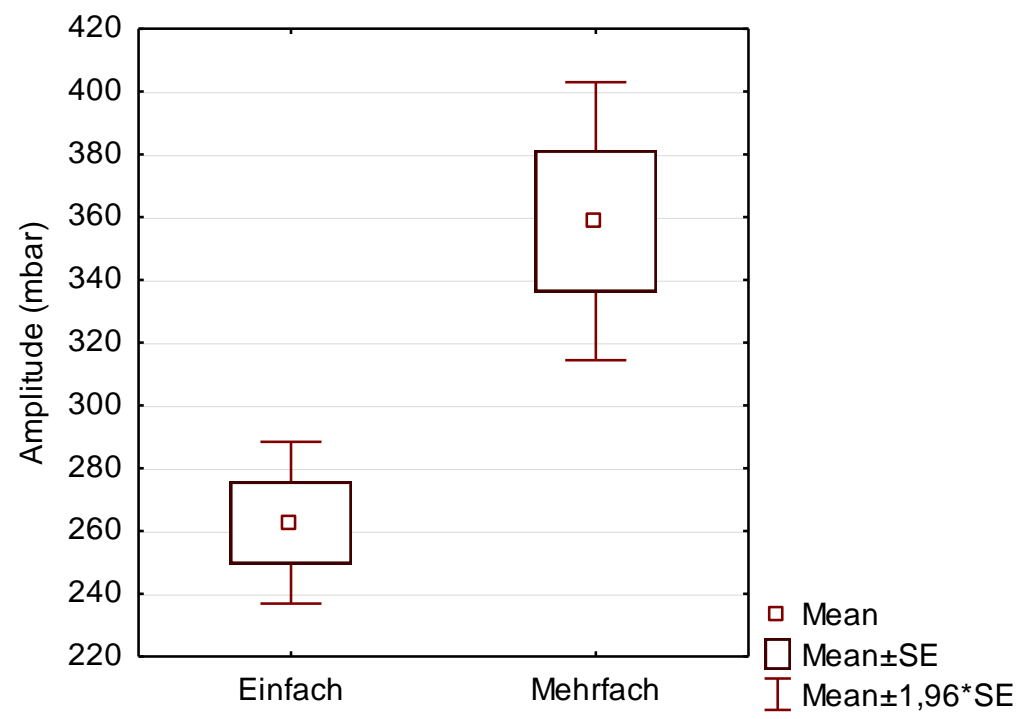

Abbildung 25 Grafische Darstellung der Verteilung der Probanden nach dem Druckaufbau und dem Mittelwert der Amplituden bei der aktiven Einnahme eines Bolus

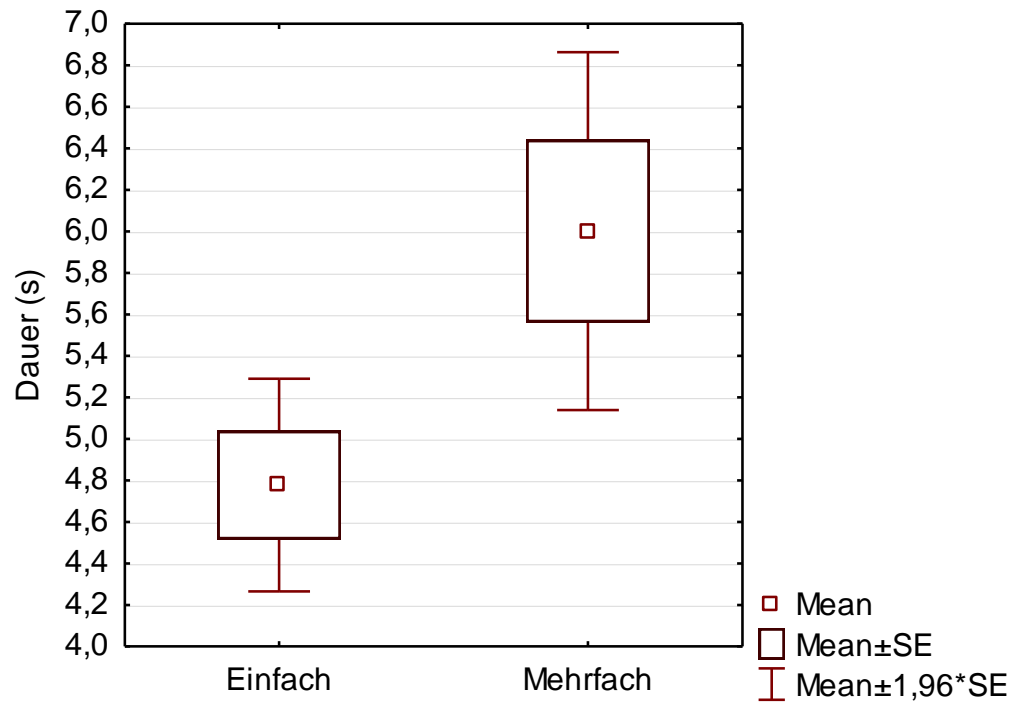

Abbildung 26 Grafische Darstellung der Verteilung der Probanden nach dem Druckaufbau und dem Mittelwert der Dauer bei der aktiven Einnahme eines Bolus

Bei der Beschreibung des Druckabfalls konnten zwei Muster erkannt werden, anhand derer die Probanden unterteilt werden konnten. 35 Probanden zeigten einen raschen Druckabfall, die restlichen 17 einen stufenförmiger Abfall. Diese Unterteilung konnte mit der Amplitude und Dauer der Ereignisse korreliert werden. Hierbei konnte beobachtet werden, dass signifikant höhere Amplituden und eine längere Dauer beim raschen Druckabfall auftraten $(\mathrm{p}<0.05)$. Weitere Angaben sind in der Tabelle 5 und in den Abbildungen 27 und 28 dargestellt. 
Eine Beziehung (McNemar Test) zwischen den Merkmalen beim Druckaufbau und Druckabfall konnte beobachtet werden. Probanden, die einen mehrfachen Aufbau hatten, zeigten meistens auch einen raschen Druckaufbau $(\mathrm{p}<0.05)$.

\begin{tabular}{lrrrrr}
\hline & MW & MW & SD & SD & p-Wert \\
& Rasch & Stufe & Rasch & Stufe & \\
\hline Amplitude & 308.4829 & 253.0176 & 103.8535 & 46.08495 & 0.010345 \\
Dauer & 5.427893 & 4.517924 & 1.789255 & 1.354122 & 0.048047 \\
\hline
\end{tabular}

Tabelle 5 Vergleich (T-Test) der beiden Merkmalen des Druckaufbaus (Stufe und Rasch) mit der Amplitude bzw. Dauer bei der aktiven Einnahme eines Bolus

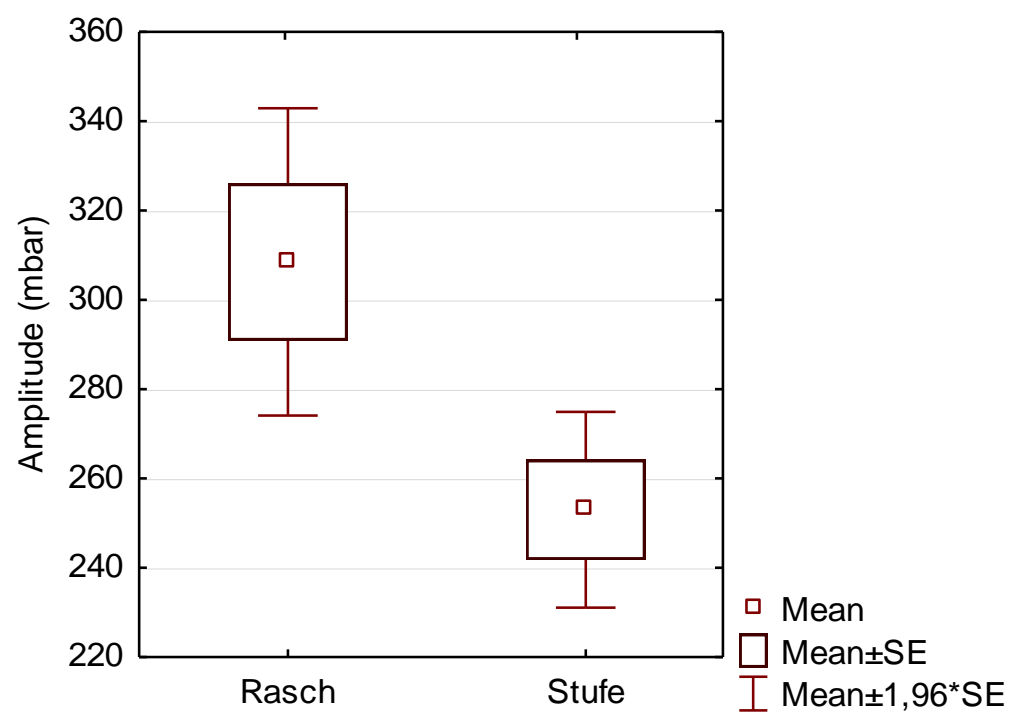

Abbildung 27 Verteilung der Probanden nach dem Merkmal des Druckabfalls und den Mittelwerten der Amplituden bei der aktiven Einnahme eines Bolus 


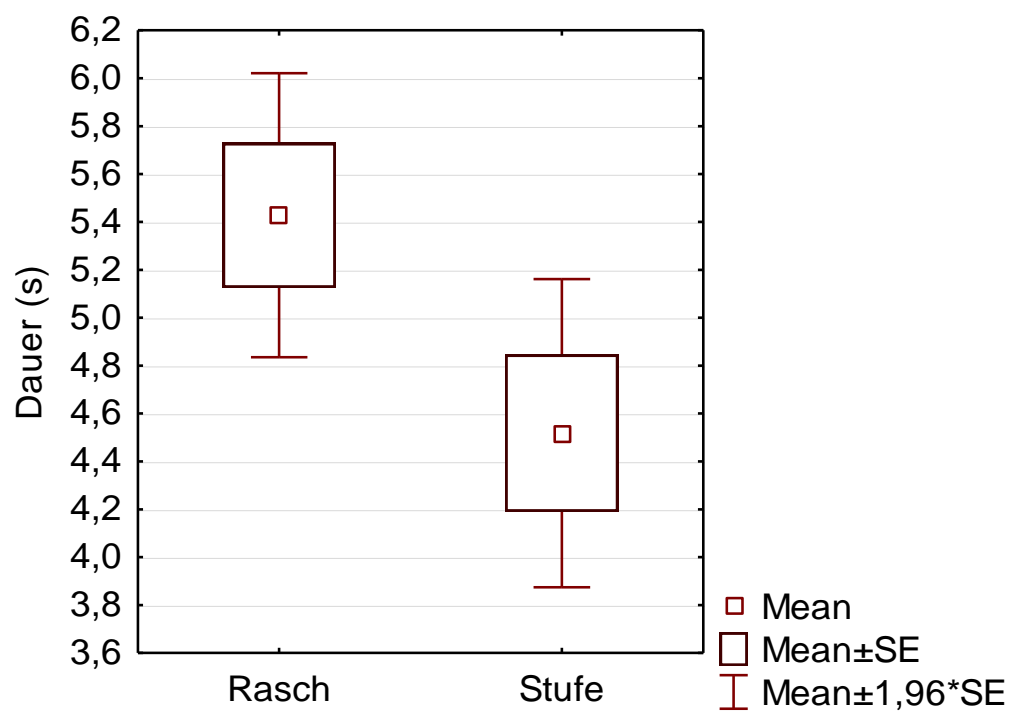

Abbildung 28 Verteilung der Probanden nach dem Merkmal des Druckabfalls und den Mittelwerten der Dauer bei der aktiven Einnahme eines Bolus

\subsubsection{Passive Wasser-Bolus-Gabe (PWG)}

Nicht nur negative Druckamplituden wurden aufgezeichnet, sondern auch Drücke im positiven Bereich, die nach der negativen Druckamplitude auftraten (siehe Abbildung 15). Bei der passiven Gabe eines Bolus aus Wasser konnte bei 6 Probanden das Auftreten von positiven Amplituden erkannt werden. Dieses Merkmal konnte mit der Amplitude und Dauer der Kurven korreliert werden. Bei denjenigen Probanden, die positive Drücke aufwiesen, wurde eine signifikant geringere Amplitude und eine kürzere Dauer beobachtet. Bezüglich der Kurvenkomplexität wurden bei der passiven Gabe eines Wasser-Bolus ausschließlich monophasische Kurven beobachtet.

Eine Zusammenfassung der Korrelation der positiven Druckverläufe und der Amplituden beziehungsweise der Dauer ist in der Tabelle 6 dargestellt und in den Abbildungen 29 bis $30 \mathrm{zu}$ erkennen. 


\begin{tabular}{lccccc}
\hline & MW & MW & SD & SD & p-Wert \\
& Ja & Nein & Ja & Nein & \\
\hline Amplitude & 14.33333 & 33.98043 & 11.30321 & 23.75453 & 0.005367 \\
Dauer & 1.085847 & 1.957900 & 0.604862 & 1.245188 & 0.015441 \\
\hline
\end{tabular}

Tabelle 6 Vergleich (T-Test) der beiden Merkmale $(\mathrm{Ja}=$ Auftreten von positiven Druckamplituden; Nein= Kein Auftreten von positiven Druckamplituden) mit Amplitude bzw. Dauer bei der passiven Gabe eines Bolus aus Wasser

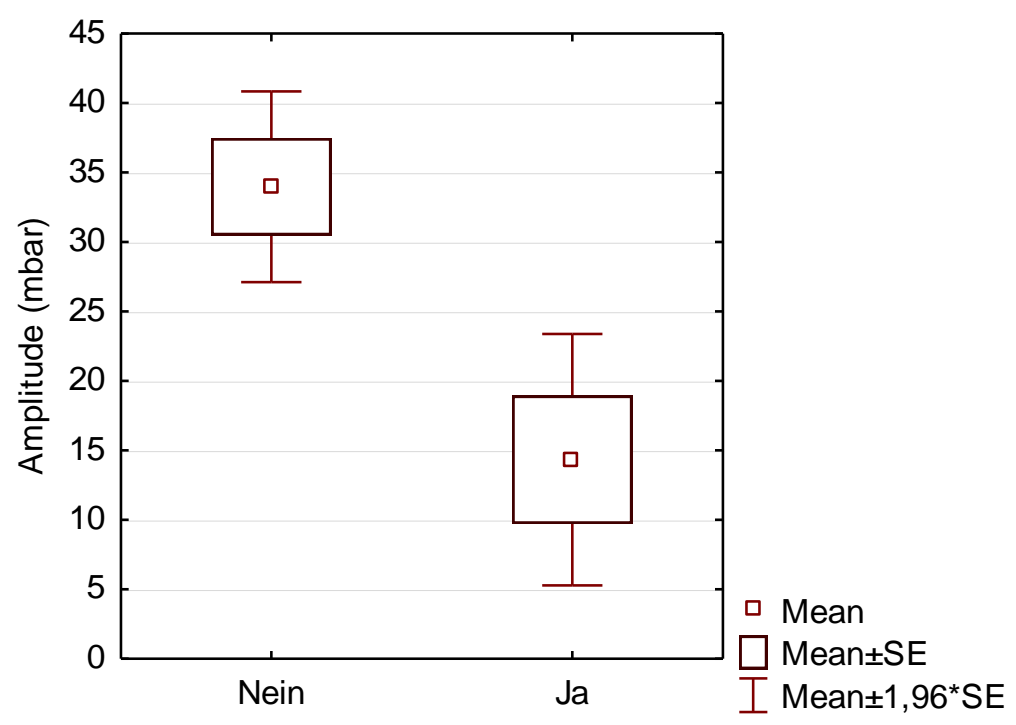

Abbildung 29 Verteilung der Probanden nach dem Auftreten von positiven Druckamplituden und den Mittelwerten der Amplituden bei der passiven Gabe eines Bolus aus Wasser. Ja= Auftreten von positiven Druckamplituden; Nein= Kein Auftreten von positiven Druckamplituden 


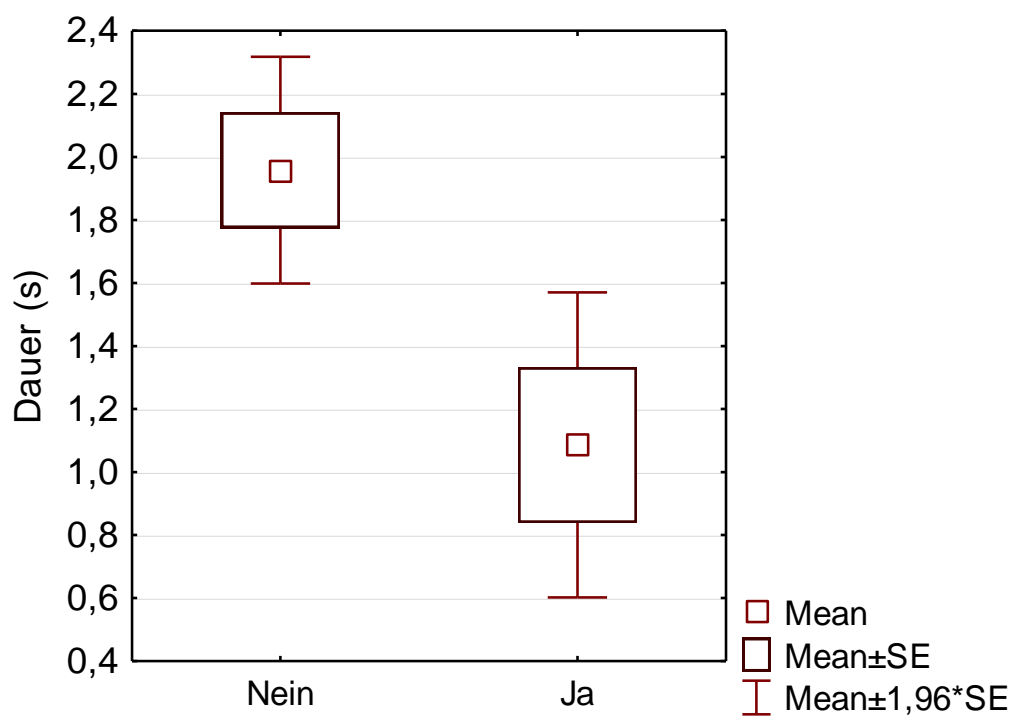

Abbildung 30 Verteilung der Probanden nach dem Auftreten von positiven Druckamplituden und den Mittelwerten der Dauer bei der passiven Gabe eines Bolus aus Wasser

\subsubsection{Passive Gel-Bolus-Gabe (PGG)}

Bei der passiven Applikation eines Bolus aus Gel konnten auch positive Druckamplituden erkannt werden. Diese konnten bei 40 Probanden festgestellt werden. Bei diesen Probanden wurde auch tendenziell erkannt, dass das Auftreten von positiven Druckamplituden mit einer niedrigeren Amplitude des Schluckvorganges und einer kürzeren Dauer der Kurve verbunden war, was aber statistisch nicht zu belegen war. Genauer beschrieben ist diese Korrelation in der Tabelle 7 und in den Abbildungen 31 und 32.

\begin{tabular}{lccccc}
\hline & MW & MW & SD & SD & p-Wert \\
& Ja & Nein & Ja & Nein & \\
\hline Amplitude & 36.54634 & 38.96364 & 29.18847 & 30.09552 & 0.814977 \\
Dauer & 1.431532 & 1.939245 & 0.974061 & 1.157993 & 0.203738
\end{tabular}

Tabelle 7 Vergleich (T-Test) der beiden Merkmale ( $\mathrm{Ja}=$ Auftreten von positiven Druckamplituden; Nein= Kein Auftreten von positiven Druckamplituden) mit der Amplitude bzw. Dauer bei der passiven Gabe eines Bolus aus Gel 


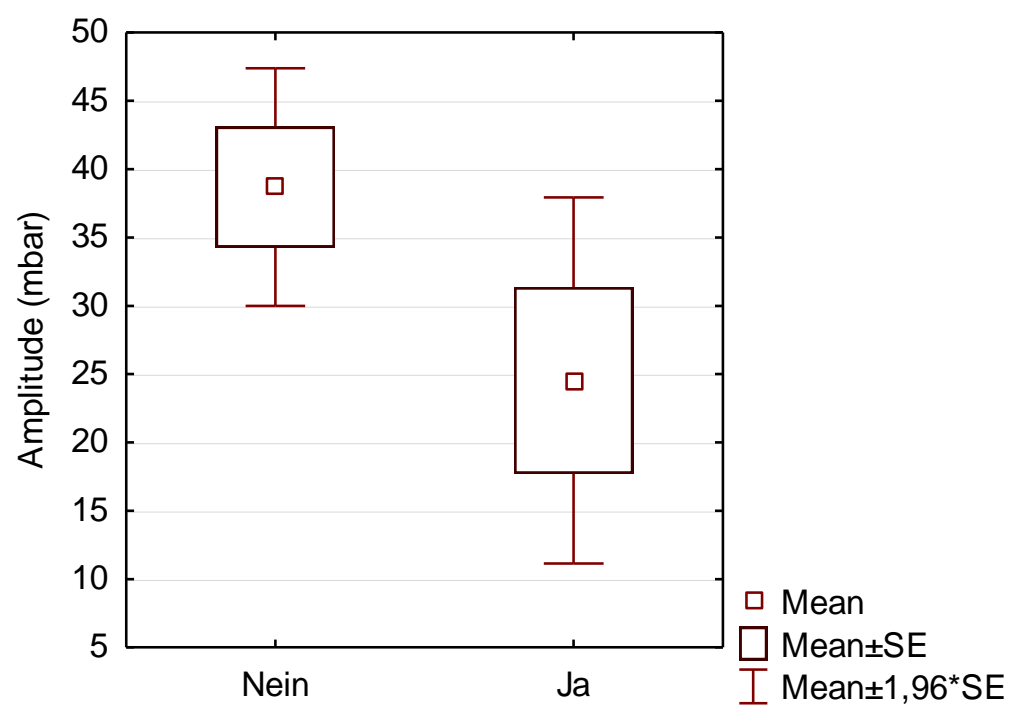

Abbildung 31 Verteilung der Probanden nach dem Auftreten von positiven Druckamplituden und den Mittelwerten der Amplituden bei der passiven Gabe eines Bolus aus Gel

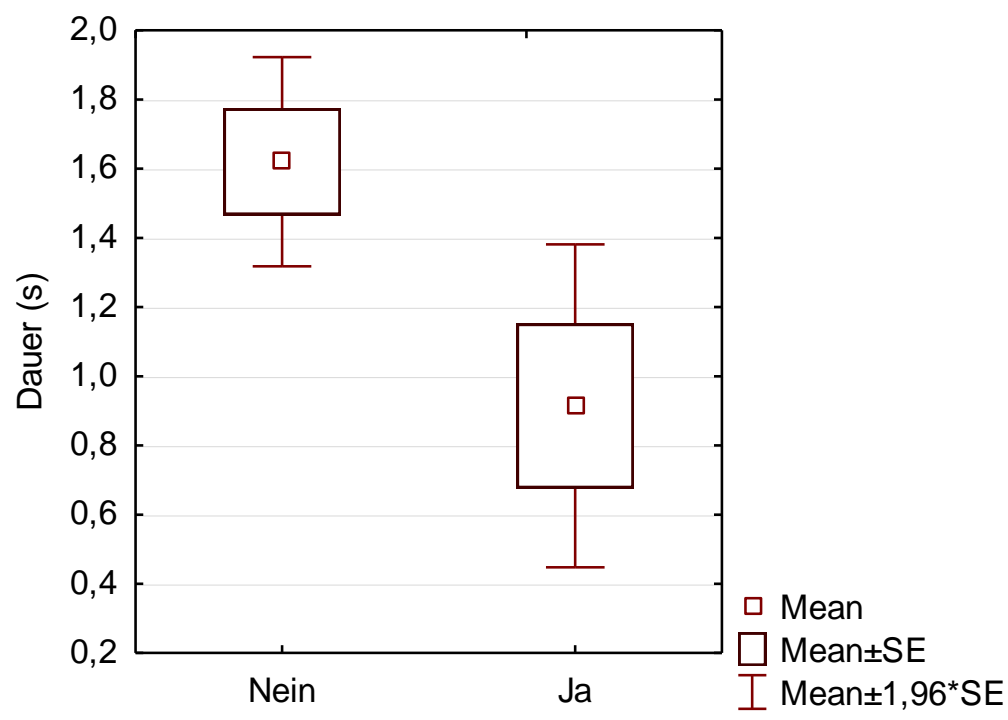

Abbildung 32 Verteilung der Probanden nach dem Auftreten von positiven Druckamplituden und den Mittelwerten der Dauer bei der passiven Gabe eines Bolus aus Gel

Bezüglich der Kurvenkomplexität wurde bei der Gabe eines Gel-Bolus bei 35 von den 52 Probanden eine polyphasische Kurve erkannt, die durch einen komplexen M-artigen Verlauf gekennzeichnet war. Die restlichen 17 Probanden zeigten eine monophasische Kurve. 
Dieses qualitative Merkmal konnte mit der Amplitude und der Dauer korreliert werden. Die Amplitude der polyphasischen Kurven bei der Gabe eines Bolus aus Gel war tendenziell höher, erwies sich aber nicht als signifikant. Dies war auch der Fall bei der Dauer, wo bei Probanden mit einer polyphasischen Kurve eine tendenziell längere Dauer der Vorgänge erkannt werden konnte. Genauer beschrieben werden diese Ergebnisse in der Tabelle 8.

\begin{tabular}{llllll}
\hline & \multicolumn{1}{c}{$\begin{array}{c}\text { MW } \\
\text { Monophasisch }\end{array}$} & \multicolumn{1}{c}{ MW } & \multicolumn{1}{c}{ SD } & SD & p-Wert \\
& Polyphasisch & Polyphasisch & Monophasisch & \\
\hline Amplitude & 26.55882 & 42.15714 & 33.91578 & 25.43636 & 0.105277 \\
Dauer & 1.271818 & 1.668674 & 0.752077 & 1.121251 & 0.138411 \\
\hline
\end{tabular}

Tabelle 8 Vergleich (T-Test) der beiden Merkmale (Monophasisch und Polyphasisch) mit der Amplitude bzw. Dauer bei der passiven Gabe eines Bolus aus Gel

Die in der Tabelle vorgestellte Verteilung der Probanden ist in der Abbildung 33 bezüglich der Amplitude und in der Abbildung 34 bezüglich der Dauer anhand von Box-Plots grafisch dargestellt.

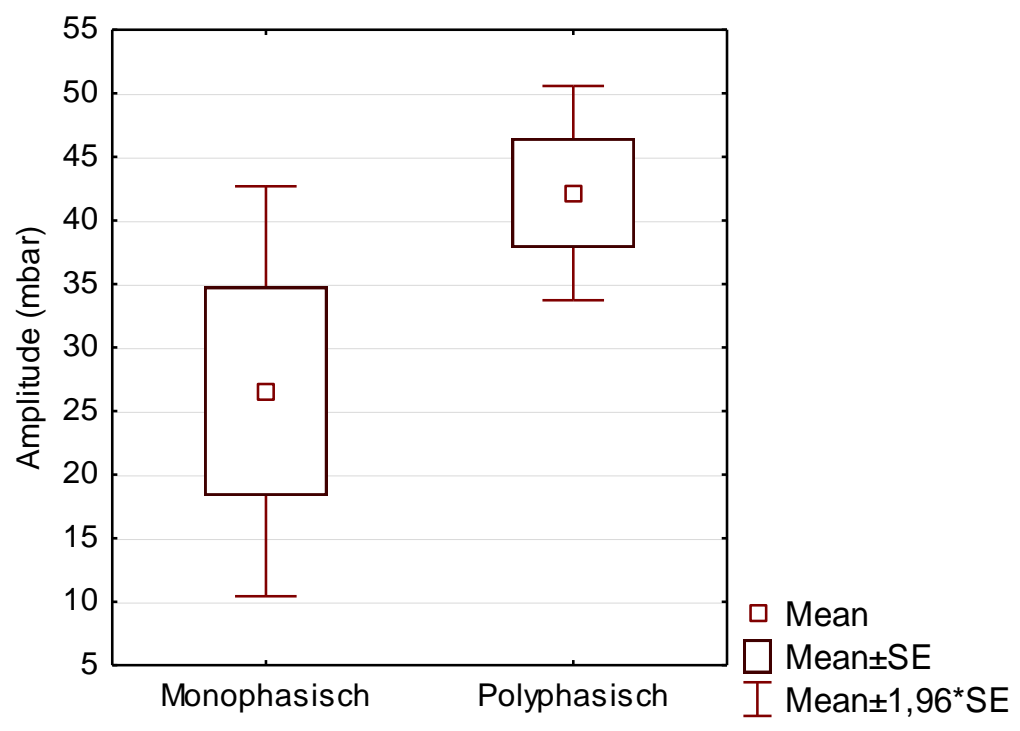

Abbildung 33 Verteilung der Probanden bezüglich der Kurvenkomplexität und der Mittelwerte der Amplituden 


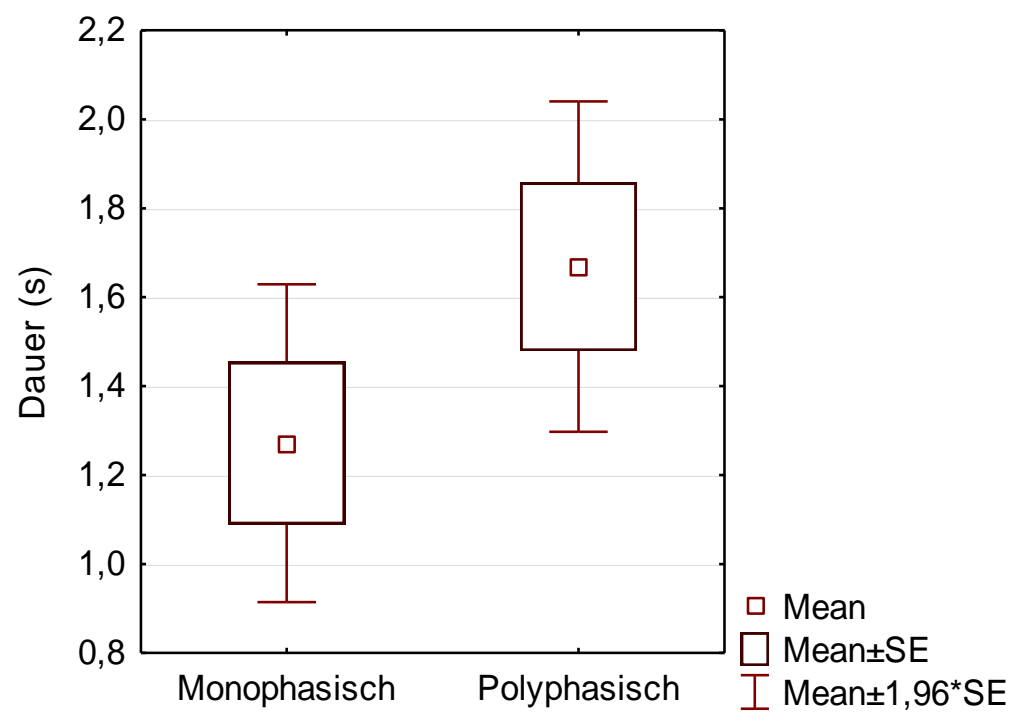

Abbildung 34 Verteilung der Probanden bezüglich der Kurvenkomplexität und des Mittelwertes der Dauer 


\section{Diskussion}

Schlucken ist eine lebensnotwendige Funktion, die eine vollständige Ernährung und Hydratation ermöglicht. Dieser hochkomplexe neuromuskuläre Prozess gewährleistet den Transport des Bolus in den Magen und sichert zugleich den Luftweg (Langmore et al. 1998; Marik und Kaplan 2003). Besonders im Alter ist es wichtig, Schluckstörungen frühzeitig zu erkennen und zu behandeln, da sie Aspirationspneumonien verursachen können (Martin-Harris et al. 2005). Allgemein kann gesagt werden, dass Schluckstörungen im Alter häufiger auftreten (Feinberg et al. 1990). Laut einer epidemiologischen Studie aus dem Jahr 2004 liegt die Prävalenz von Schluckstörungen älterer Personen (älter als 65 Jahre) bei 13.8\% (Gleeson 1999; Kawashima et al. 2004).

Die Beurteilung des Schluckvorganges unterliegt verschiedenen Herausforderungen. Dazu gehört unter anderem die funktionelle Untersuchung bei geschlossener Mundlage, was die klinische Bewertung deutlich erschwert. Andererseits ist auch bei dem methodischen Aufbau zu beachten, dass die strukturellen und physiologischen Abläufe nicht behindert werden. So wurde in den letzen Jahren nach einfach durchzuführenden Methoden geforscht, die gleichzeitig sicher, röntgenhygienisch und kostengünstig sind (Ono et al. 2004; Tamine et al. 2010). 


\subsection{Angewendete Methode}

Die Untersuchung des Schluckaktes mittels Druckmessung hat im letzten Jahrzehnt eine aufsteigende Entwicklung erlebt. Die manometrische Untersuchung zeichnet sich durch die Ermittlung ausführlicher Informationen zur Zungenkraft, Koordination und Funktion aus, ohne dabei den Patienten mit der Einnahme von Kontrastmitteln oder Röntgenbestrahlung zu belasten (Miller A 1982). Gleichwohl ist zu beachten, dass eine bildgebende Darstellung der anatomischen Strukturen mittels alleiniger Druckmessungen nicht gegeben ist, so dass die erhobenen manometrischen Daten immer eine Interpretation verlangen. Zudem sind die Ergebnisse vom methodischen Abbau abhängig.

In der vorliegenden Arbeit wurden drei verschiedene Schluckuntersuchungen mit Änderungen der Boluskonsistenz und Applikationsart durchgeführt. Als Messort und Stelle für die Bolusgabe wurde der subpalatinale Raum gewählt - bezogen auf das biofunktionelle Modell nach Engelke et al. (2011). Danach kann das orofaziale System in funktionelle Einheiten unterteilt werden, in denen verschiedene Verschlüsse geschlossene Kompartimente bilden können. Der Bolus verweilt kurz im subpalatinalen Raum, bevor er in den Pharynx gelangt (Engelke et al. 2011).

Wie in der von Engelke et al. zitierten Arbeit von 2011 wurde für diese Zwecke ein piezoresistives Druckmessgerät benutzt. Das von der benannten Arbeitsgruppe verwendete Mundstück wurde für die Fragestellung dieser Arbeit modifiziert, indem eine Silikonschlaufe eingebracht wurde (Engelke et al. 2011). Wie bereits in der Methodik dieser Arbeit beschrieben, erreichte die Silikonschlaufe den subpalatinalen Raum. Die Probanden schilderten keine Hindernisse während des Schluckvorganges aufgrund des Mundstückes. Auch die unterschiedliche Einnahme des Bolus (aktiv oder passiv) konnte von allen Probanden durchgeführt werden. Die Konsistenz des Bolus erwies sich als unproblematisch und während der gesamten Messung wurde keine Ablehnung des Bolus oder das Auftreten von Verschlucken registriert.

Bei dieser Arbeit wurde der Schluckvorgang unter drei verschiedenen Bedingungen erforscht. Jede dieser Bedingungen beinhaltete 10 Schluckvorgänge, so wurden insgesamt 30 Schluckvorgänge bei jedem Probanden aufgezeichnet und um die 1560 Schluckvorgänge während der gesamten Arbeit. Ziel der Wiederholung der 
Schluckvorgänge war es, die in anderen Studien bekannt gegebene hohe intraindividuelle Variabilität der oralen Phase $\mathrm{zu}$ reduzieren und so mögliche Messfehler zu vermeiden (Thüer et al. 1999; Youmans und Stierwalt 2006; Steele und Van Lieshout 2009). Mit dieser hohen Anzahl an Versuchen konnte überprüft werden, in welchem Maß diese Variabilität von den gegebenen Bedingungen abhängig ist. Auch die Erkennung des Auftretens von gewissen Schluckmustern und Merkmalen bei jedem Probanden und im Gesamtkollektiv sollte durch die Wiederholung des Tests gewährleistet sein.

Aus vorhergehenden Untersuchungen von Thüer et al. und Fröhlich et al., kann geschlossen werden das ein Messbereich von 2000 mbar bis zu -1000 mbar und ein zeitliches Auflösungsvermögen von $1 \mathrm{kHz}$ für unsere Untersuchung ausreichend ist (Thüer et al. 1985; Fröhlich et al. 1991). In Bezug auf die angewendete Methode und unter Berücksichtigung der Messanordnung und Dimension des benutzten Schlauchsystems wurde der absolute Messfehler mit 5 mbar abgeschätzt. Da die Größenordnung der gemessenen Druckwerte weit über dem Messfehler liegt, kann die hier angewendete Methode valide eingesetzt werden.

Eine Möglichkeit, den Messfehler erheblich zu vermindern, wurde von Thüer et al. und Fröhlich et al. vorgeschlagen, die jedoch mit großem Aufwand verbunden ist. Für Fragestellungen, die eine höhere absolute Messgenauigkeit erfordern, kann diese Methode auch für unsere Zwecke erwogen werden. 


\subsection{Interpretation der erhobenen Kurven}

\subsubsection{Die aktive Bolus-Einnahme - manometrische Darstellung der Saugfunktion}

Bei der Untersuchung der aktiven Bolus-Einnahme wurden die Probanden aufgefordert, Wasser aus einer Spritze zu saugen. In diesem Versuch wurde angestrebt, dass die Probanden die eigene natürliche Saug- und Schluckaktivität reproduzieren. Die Probanden konnten ein freigegebenes Volumen an Wasser aufnehmen, bis der Schluckvorgang möglich war. Diese Freigabe der Saugfunktion wurde auch in der Arbeit von Nilsson et al. (1996) beschrieben, in der die Probanden einen Strohhalm bekamen, mit dem sie Wasser aus einem Becher saugen konnten. Das angesammelte Volumen an Wasser wurde anschließend von den Probanden geschluckt, wie es bei unserer Versuchsanordnung auch der Fall war.

Bei der Beobachtung unserer Ergebnisse konnten wir feststellen, dass bei der aktiven Einnahme eines Bolus im Vergleich zur passiven Gabe höhere Druckamplituden mit einem Mittelwert von -290 mbar auftraten.

Untersuchungen zur Saugfunktion bei Erwachsenen sind nicht verbreitet und in der wissenschaftlichen Literatur kaum vertreten. Beim Säugling dagegen steht die Saugfunktion im Fokus verschiedener Untersuchungen, da der Saugmechanismus die Basis der kindlichen Ernährung darstellt. Noch offen ist die Diskussion, wie der Milchgewinn aus der Brustwarze verläuft und ob der Milchstrahl einer Bewegung der Zunge folgt oder entlang eines Vakuums verläuft.

Saugen ist ein primitiver Reflex, der zur normalen neurologischen Antworten des gesunden Säuglings gehört. Dieser geht mit der altersbedingten Entwicklung des Kindes verloren (Schott und Rossor 2003). Im Vergleich zum Kind oder Erwachsenen sind beim Säugling anatomische Gegebenheiten wichtig, wie die unbezahnten Kieferkämme und der Kontakt zwischen Gaumen und Epiglottis. Dieser Kontakt zwischen beiden Strukturen bildet eine Verbindung, die mit dem Wachstum des Kindes verschwindet. Der Hals verlängert sich und der Pharynx dient als gemeinsamer Weg für Speise und Luft. Diese physiologischen und anatomischen Veränderungen erlauben andererseits auch die normale Entwicklung der Sprache (Matuso und Palmer 2008). 
Eine der ersten Untersuchungen zur Nahrungsaufnahme bei Säuglingen wurde von Ardran et al. im Jahre 1958 durchgeführt. Sie untersuchten mittels Videofluorskopie 35 Säuglinge bei der Einnahme von Barium-Milchpräparaten aus einer Trinkflasche (Ardran et al. 1958). Nach der Beurteilung der Bilder kamen sie zu der Auffassung, dass die Säuglinge beim Trinken rhythmische Bewegungen des Unterkiefers durchführten. Sie beobachteten ebenfalls die Lage des Schnullers zwischen der Zunge und dem Alveolarfortsatz. Seine Lage änderte sich während des ganzen Prozesses nicht. Die Zungenbewegungen beschrieben sie als ein „Rollen“ und „Pressen“, das auf den Schnuller ausgeübt wurde und so den Milchgewinn erzeugte (Ardran et al. 1958).

Es ist wichtig zu erwähnen, dass bei dieser Studie keine manometrischen Verläufe untersucht worden sind. Eine der frühsten Druckaufzeichnungen bei Säuglingen wurde von der Arbeitsgruppe von Mathew et al. (1985) bei 19 Säuglingen durchgeführt. Sie setzten einen Drucksensor im Schnuller während der Flaschenernährung ein. Hauptsächlich fokussierten sie die Untersuchung auf die Koordination zwischen Nahrungsaufnahme und Atmung, indem sie die Aufzeichnungen des Luftstromes und der Brustkorbbewegungen beurteilten (Mathew et al. 1985). Detaillierter wurden Ergebnisse zu manometrischen Schwankungen in der Mundhöhle des Säuglings im Jahre 1990 bekannt gegeben mit der Studie von Selley et al. (1990). Sie nutzten einen umfangreicheren methodischen Aufbau - Druckmessungen ergänzt mit Videofluoroskopie, Geräuschregistrierung, Ausdehnungsregistrierung des Unterkiefers und Luftströmung. Ähnlich zu unserer Untersuchung benutzten Sie eine $20 \mathrm{ml}$ Spritze als Reservoir für die Flüssigkeitsgabe. Mit dieser Messanordnung konnten sie bestätigen, dass beim Säugling negative Drücke mit einer maximalen Amplitude von 49 mbar im Mund erzeugt werden. Diese negativen Druckverläufe, wie es auch unsere Untersuchung zeigen konnte, dominierten das intraorale Milieu, da positive Druckverläufe während der aktiven Aufnahme von Flüssigkeit nicht beobachtet worden sind. Selley et al. berichteten auch von einer Korrelation zwischen negativen Druckpeaks und dem Milchfluss aus der Spritze. Dieses Ereignis konnte auch bei unseren erwachsenen Probanden erkannt werden, da sie den Spritzeninhalt durch Saugimpulse entleeren konnten. Peristaltische Bewegungen der Zunge konnten von Selleys Arbeitsgruppe auch beobachtet werden, die allerdings auf die Fixation des Schnullers im Mund und nicht mit einer effektiven Aktivität der Milchextraktion zurückgeführt worden sind (Ardran et al. 1958; Selley et al. 1990). 
Eine ausführlichere Beschreibung der Säuglingsernährung mit besonderem Augenmerk auf die Rolle der Zunge wurde im Jahre 1992 von der Arbeitsgruppe von German et al. durchgeführt. Sie implantierten radioopake Marker an der Gingiva und der Zungenoberfläche von Ferkeln und Affenjungtieren. Videofluoroskopisch verfolgten sie den Verlauf der Marker in der Zeit, in der die Tiere eine Milch-Barium-Mischung aus einer Flasche tranken, in deren Sauger ein Drucksensor platziert wurde. Sie bestätigten, dass bei der Flaschennahrung negative Drücke für den Milchfluss verantwortlich sind. Sie berichteten über rhythmische und kontinuierliche Druckschwankungen und sahen in der Saugfunktion den wichtigsten Mechanismus zur Milch-Akquisition (German et al. 1992). Diese beobachtete Regelmäßigkeit ist übereinstimmend mit unseren Ergebnissen bei der aktiven Bolus-Einnahme, während der Sogimpulse ebenso konstant durchgeführt wurden.

Weitere Angaben über Druckamplituden bei der Saugaktivität von Säuglingen wurden in der sonografischen Arbeit von Wein et al. (1993) erläutert. Auch sie beschrieben eine wellenartige, rhythmische Bewegung der Zungenoberfläche und konnten eine direkte Zuordnung zwischen Sogkurven und lingualen Bewegungen beobachten. Diese blieben bei jedem einzelnen Säugling konstant, zeigten aber im gesamten Kollektiv eine große interindividuelle Variabilität. Die Druckamplitude der Säuglinge erreichte einen Peak von bis zu -65 mbar, ein Wert, der deutlich geringer als unsere Ergebnisse bei der aktiven Bolus-Einnahme ist. Dieser Unterschied könnte durch das differente Alter der Probanden sowie durch den alternativen Versuchsaufbau erklärt werden (Wein et al. 1993).

Die Saugaktivität Erwachsener wurde von Nilsson et al. im Jahre 1996 mit dem sogenannten ROSS-Test (Repetitive Oral Suction Swallowing Test) untersucht. Sie führten den ROSS-Test bei 292 gesunden volljährigen Probanden durch. Die Versuchsanordnung umfasste akustische Messungen an der Kehlkopfregion, Bewegungsregistrierungen am Hals und Volumenberechnungen am Wasserbehälter. Der Drucksensor wurde am proximalen Ende des Strohhalmes fixiert und die Probanden wurden aufgefordert, Wasser aus einem Becher mit einem Strohhalm zu saugen und zu schlucken. Sie teilten den Test in zwei Stufen ein. Erst wurde ein „normaler“ Vorgang untersucht, hierbei fanden sie subatmosphärische Druckamplituden in einer Größenordnung von bis zu -239 mbar, die mit dem Ansammeln von Wasser korreliert werden konnte. Bei der zweiten Stufe des ROSS-Tests wurden die Probanden gebeten, 
repetitive Saug- und Schluckakte zu wiederholen bis der Becher entleert war (Nilsson et al. 1996).

Wie bei unserer Untersuchung konnten sie im zweiten Teil des Tests einen repetitiven und kontinuierlichen Verlauf erkennen, mit Druckamplituden die einem Mittelwert von -195 mbar erreichten. Die Werte im negativen Bereich von Nilsson et al. haben eine ähnliche Größenordnung wie der in unserer Studie beschriebene Mittelwert von -290 mbar. Manche Unterschiede könnten durch die Versuchsanordnung bedingt sein. Hierbei ist das größere Lumen des Strohhalmes im Vergleich zum kleineren Lumen der Silikonschlaufe zu beachten. Einen Unterschied gab es auch in der Wahl des Behälters. Beim ROSS-Test tranken die Probanden aus einem Becher, während in unserer Untersuchung eine Spritze als Flüssigkeitsbehälter genutzt wurde. Die Probanden mussten dabei die Reibung des Kolbens gegen die Wände der Spritze beim Ansaugen überwinden (Nilsson et al. 1996).

Bezüglich des Druckverlaufs konnte eine Ähnlichkeit zwischen den Druckprofilen erkannt werden. Analog zur Arbeit von Nilsson et al., in der ein rascher Aufbau von negativem Druck beobachtet wurde, konnten wir diese Morphologie auch bei der aktiven Einnahme eines Bolus erkennen. Im Gegensatz zur Arbeit von Nilsson et al. wurden in der vorliegenden Versuchsreihe verschiedene Kurvencharakteristika in der Darstellung von Druckaufbau und Druckabfall erkannt und beschrieben (Nilsson et al. 1996).

In unserer Arbeit konnten wir bei 28.8\% der Probanden einen Druckaufbau beobachten, der eine mehrfache Anzahl an Peaks zeigte, bevor ein Druckmaximum erreicht wurde. Dies kann als die Notwendigkeit mancher Probanden interpretiert werden, mehrere Saugversuche durchzuführen, bis das angemessene Volumen an Wasser angesammelt werden konnte. Die restlichen Probanden wiesen einen einfachen Druckaufbau auf, bei dem ein Sogimpuls genügte, um ausreichend Wasser zu sammeln. Der Druckabfall verlief bei den meisten Probanden (67.3\%) sehr schnell. Bei den restlichen Probanden konnte ein stufenartiges Druckgefälle beobachtet werden. Diese morphologische Gegebenheit könnte auf zwei Phasen des Schluckens hinweisen, die hypothetisch zur Vorbereitungsphase und zur reinen oralen Phase zugeordnet werden könnten.

Zur Dauer der Druckimpulse lässt sich folgende Aussage treffen: Während der aktiven Einnahme eines Bolus zeigte sich eine Tendenz zur Verkürzung der Dauer innerhalb der 
Kurvenaufzeichnung. Da dieser Test eine Herausforderung für die Probanden darstellte, könnte dieser zeitliche Trend eine Erschöpfung der Probanden andeuten. $\mathrm{Zu}$ beachten sind hierbei die beteiligten Strukturen, die bei der Saugfunktion aktiviert werden, wie das Gaumensegel und der Mundboden, die gezielt bei einer Schluckstörung mit diesem Test angesprochen werden könnten.

\subsubsection{Die passive Gabe eines Bolus}

Bei der passiven Gabe wurde der Schluckakt nach der Applikation von zwei verschiedenen Bolusarten untersucht. Die Gabe eines Bolus aus Wasser gehört zum Standard bei Schluckuntersuchungen, da Wasser das lebenswichtigste Nahrungsmittel ist, ein neutralen Geschmack und eine allgemeine Akzeptanz besitzt. Schluckuntersuchungen mit Wasser bei gesunden Probanden und bei Patienten führen auf diese Notwendigkeit zurück. Ausnahme sind bildgebende Untersuchungen, bei denen die Anwendung von Kontrastmitteln wie Barium notwendig ist (McConnel et al. 1988b; Morinière et al. 2008; Panebianco et al. 2010).

Bei der Gabe eines gelartigen Bolus wurde angedicktes Wasser verwendet, das zur Geschmacksverbesserung mit Orangenaroma versetzt worden war. Änderungen bei der Konsistenz des Bolus sind nicht nur hilfreich um den Schluckvorgang erweitert zu untersuchen, sondern auch eine häufig benutzte Alternative bei der Ernährung dysphagischer Patienten (Lazarus et al. 1993). Dies wird vor dem Hintergrund angewendet, dass ein viskoses Material während des Schluckvorganges langsamer und kontrollierter geführt wird (Hiss et al. 2004; Taniguchi et al. 2008).

Das Volumen der passiven Bolusgabe wurde auf $2 \mathrm{ml}$ festgelegt, was auf das Ziel der Arbeit zurückweist. In diesem Sinne ist zu entscheiden, ob ein kräftiges oder ein natürliches subtiles Schlucken reproduziert werden soll. Bei vorherigen Untersuchungen des Schluckaktes sind Bolusmengen von Speichelschlucken bis zu größeren Volumen von $30 \mathrm{ml}$ Flüssigkeit zu finden (Youmans und Stierwalt 2006; Felton et al. 2007; Santamato et al. 2009). In der vorliegenden Arbeit wurde der Schluckakt untersucht, wobei keine zusätzliche Anstrengung der Probanden auftreten sollte. Appliziert wurde ein minimales Volumen - ausreichend, um den normalen, nicht forcierten Schluckvorgang auszulösen (Dodds 1989). 
Bei der passiven Gabe des Bolus konnten in der vorliegenden Untersuchung vorwiegend negative Drücke aufgezeichnet werden, die im Vergleich zur aktiven BolusEinnahme deutlich geringer waren. Das Auftreten von negativen Druckverläufen bei einer passiven Gabe eines Bolus, bei der kein aktiver Saugimpuls verlangt wird, ist in der wissenschaftlichen Literatur nicht oft beschrieben. Es wird selten erwähnt und als ein Nebenbefund sekundär zu den Kontaktdrücken der Zunge genannt (Böhme, 2003).

Die erhobenen Mittelwerte der Druckamplituden bei der passiven Gabe eines Bolus unterschieden sich nicht signifikant zwischen Wasser und Gel (-31 mbar während der Wassergabe zu -37 mbar während der Gelgabe). Diese Ähnlichkeit bei den erhobenen Druckamplituden könnte vom Volumen abhängig sein, da es sich in beiden Fällen um einen 2-ml-Bolus handelte (Taniguchi et al. 2008). Andererseits konnte ein wichtiger Unterschied bei der Morphologie der erhobenen Kurven beobachtet werden, wo das Schlucken von Wasser ein deutlich einfacheres Druckprofil aufwies.

Die von uns erhobenen Ergebnisse unterschieden sich stark von den klassischen manometrischen Messungen, die sich mit dem Schluckakt befassen. In der Regel wird von positiven Druckverläufen berichtet, die aufgrund methodischer Unterschiede oder Besonderheiten des Kollektivs voneinander abweichen (Lazarus et al. 1993; Ono et al. 2004; Youmans und Stierwalt 2006). Intraorale negative Druckverläufe fielen in der Vergangenheit wegen technischer Gegebenheiten und fehlender Nutzung von Drucksensoren oft nicht auf. Intraorale Veränderungen im negativen Bereich wurden auch öfters als Messfehler interpretiert (Shellhart et al. 1997). Positive Druckverläufe weisen auf Kontaktdrücke zwischen zwei Strukturen hin, die nach einer Kraftanwendung entstehen. Dies geschieht zum Beispiel während des Schluckvorgangs, wenn die Zungenkraft gegen den harten Gaumen wirkt (Miller J und Watkin 1996).

Die ausgeübte Kraft und die Auswirkung der Bewegungen der Zunge am Gaumen werden wegen ihres wellenartigen Verlaufs öfters Peristaltik genannt (Cook et al. 1989; Chi-Fishman 2005; Steele und Van Lieshout 2008). Verschiedene Untersuchungen zeigen, dass die Zungenkraft im Verlaufe des Alters abnimmt und ihre Bewegungen langsamer werden (Youmans und Stierwalt 2006; Utanohara et al. 2008; Steele und Van Lieshout 2009). Diese Reduktion der Zungenkraft im Alter wird als Risikofaktor für Schluckstörungen erwähnt (Robbins et al. 1995). 
Differenzen zwischen der Zungenkraft von Frauen und Männern wurden untersucht und umfangreich diskutiert. Hierbei unterstützen die meisten Autoren die Meinung, dass Männer größere Kräfte beim Schlucken ausüben als Frauen (Youmans et al. 2009; Steele und Van Lieshout 2009; Clark und Solomon 2011). Bei dysphagischen Patienten konnte erkannt werden, dass beim Schlucken die Zungenkraft reduziert ist und die Zungenbewegungen unkoordiniert sind (Lazarus und Logemann 1988; Konaka et al. 2010).

$\mathrm{Da}$ in unserer Studie überwiegend negative Kompartimentdrücke registriert wurden, auch während der passiven Gabe eines Bolus, sind Kontaktdrücke nicht die einzigen Parameter, die in der Untersuchung der Schluckphysiologie erfasst werden müssen. Aus physikalischer Sicht entstehen negative Druckverläufe bei einer Reduktion des Druckes in einem geschlossenen Raum (Hoffman et al. 1998). Für intraorale negative Druckverläufe gilt dieses Gesetz auf gleiche Weise.

In dieser Hinsicht muss der Messort genau beachtet werden. Da wir im subpalatinalen Raum gemessen haben, sind dort nach dem biofunktionellen Modell nach Engelke Veränderungen des Kompartimentdruckes zu erwarten. Die Relevanz dieses Raumes liegt darin, dass der Bolus nach der Vorbereitungsphase immer dorthin gelangt. Dies ist unabhängig davon, ob es sich hierbei um einem Schneidezahntyp handelt, der den Bolus am Zungenrücken positioniert, oder um einen Schöpflöffeltyp, der den Bolus im Mundbodenbereich lagert. In beiden Fällen führt die Zunge den Bolus in den subpalatinalen Raum (Cook et al. 1989; Dodds et al. 1989). Nach dem biofunktionellen Modell bildet der Zungenrand einen hufförmigen Verschluss mit dem harten Gaumen an der lingualen Seite des Alveolarfortsatzes (palatolingualer Verschluss, V2). Der posteriore Verschluss wird durch den Kontakt zwischen Zungenrücken und weichem Gaumen gegeben (velolingualer Verschluss, V3). Diese Haltung der Zunge wurde oft als löffelartig bezeichnet, da der Bolus in dieser Grube sicher gehalten wird (Miller A 1982). Der subpalatinale Raum ist funktionell bedingt. Löst sich der velolinguale Verschluss, so konfluiert der subpalatinale mit dem angrenzenden mesopharyngeale Raum, der Bolus tritt in den Pharynx über und hiermit ist die orale Phase beendet.

Die Bedeutung der funktionellen Verschlüsse liegt nicht nur im oralen Transport begründet, sondern auch in anderen orofazialen Funktionen, wie der Lautbildung und der Atmung (Takemoto 2001; Hiiemae und Palmer 2003). Die Rolle des velolingualen 
Verschlusses wurde von Dantas et al. (1990) hervorgehoben, indem sie mittels einer kombinierten Methode von Videofluoroskopie und Manometrie den Zungengrund und den weichen Gaumen untersuchten. Sie verglichen diesen Verschluss mit der Funktion eines Sphinkters und dementsprechend benannten sie ihn ,glossopalatinalen Sphinkter“. Ein anderer Name für den Velolingualverschluss - „glossopalatinale Kontaktzone“ wurde 1996 von Kahrilas et al. (1996) geprägt.

Um den Kompartimentdruck im subpalatinalen Raum zu erzeugen, muss dieser Raum während des Schluckvorganges geschlossen bleiben, was mit einer kompetenten Aktivität der Verschlüsse gelingt. Diese Verschlüsse müssen auch dann bestehen, wenn muskuläre Kräfte den Raum ausdehnen. Nach radiografischen Beobachtungen der Arbeitsgruppe Dodds et al. wurde erkannt, dass die Zunge zur Bildung von negativen Druckamplituden beiträgt. Die Forscher beobachteten während des Schluckvorgangs eine ventrale Depression der Zunge, wobei der Zungenrand kontaktierend am Gaumen verblieb (Dodds et al. 1990).

Hypothesen zum Entstehen von negativen Druckamplituden im Mund wurden auch von Selley et al. 1990 dargestellt, als sie, wie bereits erläutert, das Schlucken bei Säuglingen erforschten. Sie beschrieben den Mundraum als eine geschlossene Kammer mit dichten Verschlüssen sowohl anterior als auch posterior. Sie erwähnten zwei Mechanismen die negative Drücke aufbauen könnten: ein leichtes Senken des Unterkiefers und die Bewegung der Zunge (Selley et al. 1990). Die Arbeitsgruppen von German und Thexton beobachteten negative Druckverläufe im Mundbereich von Ferkeln und Affenjungtieren während der Flaschenernährung. Die gleichzeitige Videofluoroskopie der Zunge erlaubte eine Korrelation zwischen der Zungenbewegung und den negativen Druckverläufen (German et al. 1992; Thexton et al. 2004). Sie beschrieben eine vertikale Bewegung des Unterkiefers und eine starke ventrale Depression der Zunge. Auch sie sprachen von einem geschlossenen Raum, bei dem die Zungenspitze anterior am Flaschenschnuller und die Zungenbasis posterior am weichen Gaumen Abdichtungen bildeten (German et al. 1992). Bei der passiven Gabe eines Bolus konnten Thexton et al. ebenfalls negative Drücke registrieren und dabei auch einen verschlossenen Raum erkennen, der anatomisch von der Zunge begrenzt wurde (Thexton et al. 2004). 
Die Rolle des weichen Gaumens bei der Entstehung von negativen Druckverläufen wurde auch mittels elektromyographischen Untersuchungen von Iida et al. (2003) dargestellt. Beim Saugen und Schlucken konnten sie den Aufbau von negativem Druck der kaudalen Bewegungen des Unterkiefers zuordnen, während ein velolingualer Verschluss bestand. Sie sind der Auffassung, dass der Musculus levator veli palatini bei dem Erhalt dieses Verschlusses beteiligt ist und der Musculus palatoglossus bei der Erzeugung des Unterdruckes (Iida et al. 2003).

Positive und negative Druckverläufe scheinen die orale Phase des Schluckvorganges zu begleiten. Ein kombiniertes Hervortreten von negativem und positivem Druck beim Schlucken wurde in den Untersuchungen von den Arbeitsgruppen von Kieser und Kennedy beobachtet (Kieser et al. 2008; Kennedy et al. 2010). Kieser et al. untersuchten im Jahr 2008 Druckveränderungen bei fünf Probanden während der passiven Gabe eines Bolus aus Wasser. Die Probanden erhielten eine individuell angefertigte Oberkieferschiene, die Drucksensoren entlang des Gaumens und am Zahnbogen enthielt. Mit dieser Schiene bekamen die Probanden $10 \mathrm{ml}$ Wasser aus einem Becher zu trinken.

Die Autoren beobachteten beim Schlucken, dass die Druckverläufe der verschiedenen Sensoren stark abwichen, boten aber für diese Beobachtungen keine Erklärung. Basierend auf dem biofunktionelle Modell ist eine Interpretation der Ergebnisse von Kieser et al. möglich. Sensoren, die ausschließlich positive Drücke zeigen, befinden sich nach dem biofunktionellen Modell in einer Ventilzone. Dies galt für den labialen Bereich, die Zungenspitze und den Zungenrand. Sensoren, die negative oder komplexe Peaks aufzeichnen, befinden sich in der Zone eines Kompartimentes. 


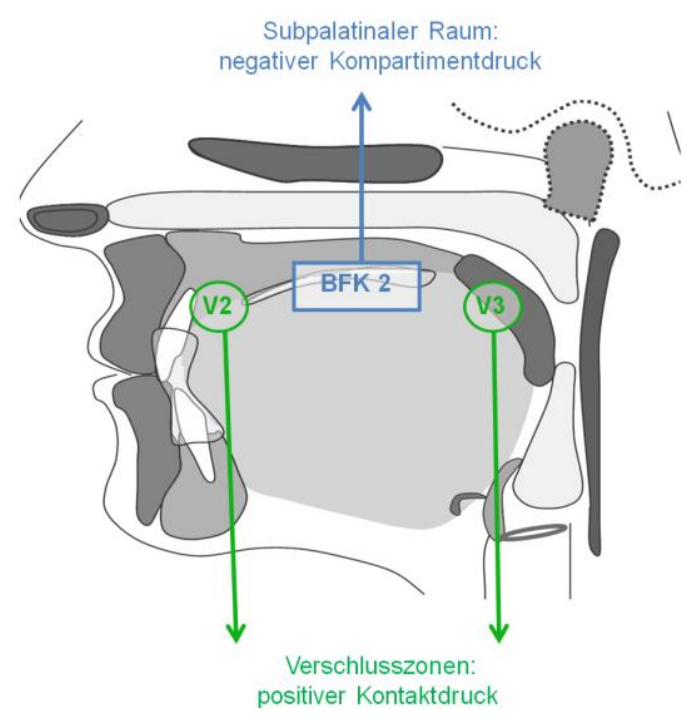

Abbildung 35 Schematische Darstellung des orofazialen Systems mit den Verschlüssen V2 - V3 und dem Kompartiment 2. Gekennzeichnet sind die Zonen, wo das Auftreten von Kontakt und Kompartimentdrücken nach dem biofunktionellen Modell erwartet wird

Negative Druckamplituden im Kompartiment deuten auf eine Ausdehnung des geschlossenen subpalatinalen Raums hin. Ein positiver Druckpeak gemessen in der Zone eines Kompartiments kann auf den Kontaktdruck zwischen den elastischen Strukturen hinweisen. Dieser kann nach der Ausbildung eines Vakuums entstehen, dann wenn die Wände eines Raumes gegeneinander kollabieren.

Kieser et al. beobachteten Druckamplituden von bis zu -180 mbar. Diese sind deutlich höher im Vergleich zu dem von uns beobachteten Mittelwert von -31 mbar bei Wasser und -37 mbar bei Gel. Dies könnte dafür sprechen, dass die Probanden bei der Flüssigkeitseinnahme gesaugt haben. Eine erweiterte Folgearbeit der erhobenen negativen Druckverläufe am Gaumen wurde von Kennedy et al. im Jahre 2010 vorgenommen. Mit der gleichen Oberkieferschiene und Versuchsanordnung untersuchten sie bei 6 gesunden Probanden Druckschwankungen an der Mittellinie des Gaumens. Sie konnten einen Druckverlauf erkennen, der unserem polyphasischen Muster bei der Gabe eines Gel-Bolus ähnelt. Dieser Verlauf beschreibt schnelle Drucksenkungen im negativen Bereich gefolgt von raschen Schwankungen im positiven und negativen Bereich. Diese morphologische Beschreibung entspricht der von uns beobachteten polyphasischen Kurven. 
Auch bei der Folgearbeit von Kennedy et al. wurde von Druckpeaks bis zu 200 mbar berichtet, ein Wert, der stark von unseren Befunden abweicht. Wichtig bei der Interpretation der Ergebnisse ist die Berücksichtigung des methodischen Aufbaus. Beide Studien verlangen von den Probanden das Trinken aus einem Becher. Dabei können individuelle Angewohnheiten beim Trinken aus einem Becher, wie das Ansaugen der Flüssigkeit, die Messung beeinflussen. Sie nutzen ein starres Gerät, das am Gaumen fixiert war und dort gemessen hat. Bei unserem Mundstück wurde durch einen flexiblen Schlauch die Mundhöhle mit einem extraoralen Sensor verbunden. Auch die Art der Bolusgabe ist eine wichtige Differenz. In unserem Versuch wurde der Bolus direkt unter den Gaumen appliziert. Bei den Arbeiten von Kieser und Kennedy wurden die Probanden beauftragt, aus einem Becher $\mathrm{zu}$ trinken. Zuletzt ist auch die Probandenanzahl und statistische Methodik zu erwägen. In unserem Probandenkollektiv von 52 Probanden wurde der Mittelwert genutzt um die Ergebnisse zu beschreiben. Sowohl in der Arbeit von Kieser wie auch in der von Kennedy sind keine Mittelwerte oder Medianwerte der insgesamt 11 Versuchspersonen gegeben. Sie erwähnten jeweils die Druckpeaks bei einem Beispielprobanden. Da insbesondere die orale Phase hohen interindividuellen Variationen unterliegt, ist dieser letzte Punkt ein sehr wichtiger Faktor, der die Ergebnisse stark beeinflussen kann.

Bezüglich der Dauer der manometrischen Druckverläufe während der passiven Gabe eines Bolus konnte bei unseren Messungen im subpalatinalen Raum eine Druckveränderung beobachtet werden, die eine mittlere Dauer von $1.8 \mathrm{~s}$ bei der WasserGabe und $1.5 \mathrm{~s}$ bei der Gel-Gabe pro Schluckvorgang aufwies. Dies befindet sich in dem von Logemann angegeben Bereich der Dauer der oralen Phase (Logemann 1998).

Bei der Gabe eines Bolus aus Wasser herrschten Kurvenprofile, die einen monophasischen Verlauf in den negativen Bereich aufwiesen, vor. Diese negativen Drücke könnten auf einen Saugmechanismus beim Transport der Flüssigkeit im Kompartiment hindeuten. Eine andere Hypothese über die Bedeutung der negativen Druckimpulse ist, dass sie bei der Auslösung des Schluckreflexes involviert sein könnten. Die Verteilung der Rezeptoren, die die Auslösung des Schluckreflexes erzeugen, ist bisher noch ein Diskussionspunkt. Traditionell wird der Arcus palatoglossus als der Bereich angesehen, wo der Schluckreflex ausgelöst wird (Pommerenke 1928). Neuere Untersuchungen deuten auf eine erweiterte Verteilung der Rezeptoren hin, die an Zunge, Epiglottis, Larynx und Uvula liegen könnten (Pereira et 
al. 2008; Takatsuji et al. 2012). Unter diesem Aspekt sind in der Schluckphysiologie noch viele Fragen offen.

Polyphasische Kurven wurden ausschließlich bei der Gabe eines Bolus aus Gel beobachtet. Diese Kurven zeichneten sich durch repetitive negative Peaks aus. Sie wiesen eine leichte Verlängerung der Dauer der Kurven und das Auftreten von positiven Druckimpulsen auf. Diese morphologische Besonderheit bei einem viskosen Bolus könnte auf eine vielseitigere Motorik der subpalatinalen Raums hindeuten. Bedingt durch die Zähigkeit des Bolus könnten für den Transport des Bolus mehrere Saug- und positive Druckimpulse notwendig sein. Andererseits könnte bei der erhöhten Aktivität die Portionierung des Bolus notwendig sein oder es zu einem Kontaktdruck der Strukturen kommen, die den subpalatinalen Raum begrenzen. 


\subsection{Darstellung des Schluckvorganges nach dem biofunktionellen Modell}

Gestützt auf den Stand der Wissenschaft (Dodds 1989; Logemann 1998) bezüglich der Strukturen im oropharyngealen Bereich kann die Vorbereitungs- und die orale Phase des Schluckvorganges anhand des biofunktionellen Modells und der erhobenen Ergebnisse beschrieben werden.

Die Vorbereitungsphase findet nach der Einnahme des Bolus statt. Sie beginnt mit der Präparation des Bolus bis $\mathrm{zu}$ einer breiartigen Konsistenz. Dies entspricht der Kaufunktion und der Befeuchtung der Speise mit Speichel. Bei fester Kost ist die Bildung von Verschlüssen nicht unbedingt erforderlich, obwohl sie öfters die Vorbereitungsphase begleitet. Der Bolus wird unter Führung der Zunge im Mund mehrere Male auf die Kaufläche und auf dem Zungenrücken positioniert. Hierbei besteht kein palatolingualer Verschluss zwischen den Funktionsräumen 1 und 2. Jedoch besteht ein dorsaler palatolingualer Verschluss zwischen den Funktionsräumen 2 und 3. Dies erlaubt eine Verbindung der interokklusalen und subpalatinalen Räume bei gleichzeitiger freier Luftpassage durch den Funktionsraum 3. Bei flüssiger Kost verliert die Vorbereitungsphase an Bedeutung, da der Bolus eine zum Schlucken geeignete Konsistenz hat und ausschließlich durch die Mundhöhle transportiert wird.

Im Rahmen unserer Untersuchungen konnte ein zentraler Vorgang des Schluckens, nämlich die Bildung eines zum Transport von Fluiden notwendigen Verschlusses mit nachfolgender Erweiterung des Funktionsraumes 2 belegt werden. Diese Verschlussbildung äußert sich als Änderung des Kompartimentdruckes im Bezug zur Umgebung. Die beim Saugen zu beobachtende mittlere negative Druckamplitude von 290 mbar konnte auch im Zusammenhang der passiven Bolusgabe beobachtet werden. Hier ist sie zwar geringer ausgeprägt (mit einem Mittelwert von -31 mbar bei Wasser und -37 mbar bei Gel), jedoch durchgehend erkennbar. Insbesondere beim höher viskosen Gel ist sie kombiniert mit positiven Druckimpulsen, so dass ein kombinierter Propulsion- und Saugmechanismus für diese Bolusart angenommen werden kann und das polyphasische Muster, der bei der Gel-Gabe zu erkennen ist, erklären. Die funktionelle Ähnlichkeit der Funktionen Saugen und Schlucken kann festgestellt werden. Der Transport eines flüssigen Bolus, der im subpalatinalen Raum stattfindet, 
gelingt offenbar durch einen Saugmechanismus und nicht durch eine Propulsion im Sinne einer Kompression.

In der oralen Phase wird der Bolus auf die Oberfläche der Zunge gebracht und dort kurz gehalten. Hierfür sind der palatolinguale sowie der velolinguale Verschluss notwendig und nach ihrem Entstehen wird der subpalatinale Raum vom interokklusalen und vom mesopharyngealen Raum getrennt. Der verschlossene subpalatinale Raum sichert den Bolus sowie die Luftwege, da in einem gesunden Zustand keine Leckage des Bolus in den Pharynx ermöglicht wird. Um die Passage des Bolus in den Pharynx zu gewährleisten, ist die koordinierte Öffnung des velolingualen Verschlusses, das Triggern des Schluckreflexes und die Sicherung der Luftwege erforderlich. Bei bestehendem palatolingualen Verschluss und negativen Druck im Kompartiment 2 kann der Bolus nicht in den interokklusalen Raum eintreten. Er wird durch den subpalatinalen Raum in aborale Richtung transportiert. Der vordere Anteil der Zunge befindet sich ventilartig am harten Gaumen. Dies kann muskulär aktiv unterstützt werden oder gegebenenfalls auch passiv erfolgen, wenn die Kompartimente geschlossen sind. Der hintere Anteil senkt sich, ohne den velolingualen Verschluss zu lösen. Der weiche Gaumen spannt sich segelartig nach kranial und dorsal auf, während der velolinguale Verschluss seine maximale Anspannung durchführt. Durch diese dorsale Erweiterung des Funktionsraumes 2 folgt der Bolus einem negativen Druckgradienten von der Rückenfläche der Zunge in Richtung Pharynx. Die weitere Anspannung des weichen Gaumens führt zum Kontakt mit der posterioren Wand des Pharynx. Somit bildet sich der velopharyngeale Verschluss (V4) aus. Der velolinguale Verschluss öffnet sich spontan mit der terminalen Anspannung des Velums nach dorsal kranial und verursacht die dorsal gerichtete Eröffnung des subpalatinalen Raumes mit Verlagerung des Bolus in den mesopharyngealen Raum. Unter Aktivierung des Constriktor pharyngis wird das Velum an den Zungenrücken reponiert und eine Regurgitation des Bolus in die Mundhöhle verhindert. Parallel bewegt sich der Kehlkopf-Hyoid-Komplex in vertikaler Richtung, die Glottis wird verschlossen. Unter diesen Bedingungen wird der mesopharyngeale Raum in allen Richtungen verschlossen. Nach der Eröffnung des oberen ösophagealen Sphinkters (V6) verlässt der Bolus den mesopharyngealen Raum mit Beginn der ösophagealen Phase. Desweiteren sinkt der Larynx, die Epiglottis richtet sich wieder auf und der velopharyngeale Verschluss löst sich. Die Zunge ruht am 
Gaumen, bildet erneut den palatolingualen sowie den velolingualen Verschluss mit einem spaltartig verbleibenden subpalatinalen Raum. 


\subsection{Klinischer Ausblick}

Die im Rahmen unserer Untersuchung durchgeführte Messung erwies sich bei allen Probanden als ein einfach und praktisch durchzuführender Test. Die Untersuchung wurde in circa 15 Minuten komplett umgesetzt, erfordert nur geringe Materialkosten und die von den Probanden verlangten Aufgaben wurden leicht verstanden und komplett erfüllt.

Somit bildet diese Untersuchung eine Grundlage für die Quantifizierung von Schluckleistung und Störungen der Schluckleistung bei verschiedenen Krankheitsbildern.

Die gewonnen Daten werden grafisch sowie numerisch wiedergegeben, wobei besonders die grafische Darstellung der Druckverläufe schnell zu interpretieren ist. Bei der aktiven Einnahme und bei der passiven Gabe sind die Schluckvorgänge übersichtlich zu identifizieren, auch die Amplitude und die Dauer des Druckimpulses. Die Wiederholung der Versuche - 10 Schluckvorgänge bei jedem Test - erlaubt es, die Konstanz und Regelmäßigkeit der Druckänderungen beim Schlucken abzuschätzen. Diese hohe Anzahl an Versuchen für jeden Probanden ermöglicht auch die Feststellung von Mittelwerten, Messfehlern sowie die Erkennung von möglichen Artefakten.

Auf der Basis dieser Untersuchung lässt sich ein sowohl in der Untersuchungseinheit als auch am Krankenbett einfach durchzuführender Test ableiten, der von seinen Anforderungen an den Untersuchenden und für den Patienten schnell zu erlernen und klar auszuwerten ist. Unter der Voraussetzung, dass die Bolusgabe automatisiert aufgezeichnet wird und die Dynamik der Boluszufuhr berücksichtigt wird, sind quantitative Erfassungen der Schluckleistungen als Ausgangsbefund ebenso wie als Verlaufsbeobachtung in einer objektiven Weise darzustellen. Hierzu wurden in der Arbeitsgruppe weitere Schritte zur Entwicklung eines Diagnostiksystems eingeleitet.

Die klinische Anwendung dieses Tests gewährt eine gezielte Untersuchung der Kompartimentbildung und eine Bewertung der Fähigkeit, einen intraoralen Druck zu erzeugen.

Bei Patienten mit habituellen Schluckstörungen können Aufzeichnungen der Druckverläufe Auskunft über die Zungenlage beim Schlucken geben und bei der 
Interpretation der Ruhezustände nach dem Schluckvorgang sowie während der Ruhelage helfen.

Eine fehlerhafte Lage der Weichteile im Mundbereich und deren Einfluss auf die Zahnstellung stellt für die kieferorthopädische Behandlung ein Problem dar. Hierbei hat die pathologische Zungenaktivität beim Schlucken erhebliche Bedeutung und trägt zu der Pathogenese von Malokklusionen bei (Peng et al. 2004; Ruan et al. 2005; Lambrechts et al. 2010). Die in der vorliegenden Arbeit vorgestellte Schluckuntersuchung kann in diesem Fall diagnostisch die Kompetenz bei der Bildung von Verschlüssen manometrisch anzeigen. Diese Information kann als Übung im Sinne eines Biofeedbacks zum Erlernen einer korrekten Zungenfunktion benutzt werden. Manometrisch kann die Bildung von konstanten negativen Drücken nachgewiesen werden, die eine geschlossene Ruhelage anzeigen. Kompensatorische Aktivität der mimischen Muskulatur kann damit korreliert und abtrainiert werden. In grafischer Form aufbereitete Daten können für die Therapie von Kindern mithilfe von Computerspielen genutzt und therapeutisch eingesetzt werden.

Bei organischen Störungen kann diese Schluckuntersuchung zu therapeutischen Zwecken eingesetzt werden. Bei dysphagischen Patienten ist oft eine Parese orofazialer Strukturen oder eine Defektsituation nach ablativen Eingriffen die Ursache der Dysphagie. Bei der Diagnostik können wichtige Informationen zum Gesamtfunktionszustand der Patienten gewonnen werden, die mit der Einnahme von verschieden Bolusarten verbunden sind. Da dysphagische Patienten oft eine gelartige Kost besser akzeptieren, ist diese Schulung der Schluckfähigkeit mit fraktionierter Bolusgabe eine Option, um eine Sondenernährung oder parenterale Ernährung zu vermeiden. Die bei der Untersuchung vorgeschlagene aktive Bolus-Einnahme kann bei dysphagischen Patienten auch als ein Leistungstest angewendet werden. Die Bildung von hohen negativen Druckamplituden erfordert funktionierende Verschlüsse und eine kompetente Zungenfunktion. Hierbei müssen Bewegungen der Zunge und des weichen Gaumens sowie die Mobilisierung von rekonstruierten Defektsituationen im Weichgewebe neu erlernt werden.

Die Anwendung von Saugimpulsen zur Auslösung des Schluckvorganges, um die beteiligten Muskeln $\mathrm{zu}$ tonisieren und $\mathrm{zu}$ koordinieren, wurde bereits von der Arbeitsgruppe von Nilsson vorgeschlagen. Sie konnten den ROSS-Test als 
Übungsverfahren anwenden und stellten bei Schlaganfall-Patienten fest, dass sie negative Druckimpulse erzeugen konnten und dass diese Drücke die Schluckfunktion unterstützen (Nilsson et al. 1998).

Wie bei dem ROSS-Test erlaubt der in dieser Arbeit vorgestellte Test auch Verlaufskontrollen mit quantitativer sowie qualitativer Auswertung der Patienten. Bei Patienten besteht immer die Möglichkeit, diesen methodischen Aufbau mit anderen Techniken zu ergänzen, da intra- und extraorale Bestandteile nicht mit einer MRTUntersuchung sowie mit einer endoskopischen oder videofluoroskopischen Aufzeichnung interferieren. Der Bolus kann beliebig geändert werden im Hinblick auf die Modifikation von Konsistenz und Inhalt. Der Bolus kann somit nach den individuellen Bedürfnissen der Patienten ausgewählt werden. Die hier vorgestellte Methodik kann die Grundlage einer systematischen Rehabilitation des Schluckvorganges auf der Basis einer kontrollierten, repetitiven und fraktionierten Bolusgabe darstellen. 


\section{Zusammenfassung}

Störungen der Schluckfunktion werden im Alter zunehmend diagnostiziert und beeinträchtigen die Lebensqualität der betroffenen Patienten sehr. Ein abnormes Schluckmuster bei Kindern und Jugendlichen kann eine pathologische Wirkung auf die Entwicklung der Zahnstellung haben.

In dieser Studie wurde bei einem gesunden Probandenkollektiv von 52 Teilnehmern (40 w; $12 \mathrm{~m}$ ) im Alter von 20 - 45 (MW: 25.48; SD:4.68) Jahren die orale Phase des Schluckaktes untersucht. Für diese Zwecke, wurde ein intraorales Mundstück angewendet (Silencos ${ }^{\circledR}$, Bredent, Senden, Deutschland), das aufgrund der Einbringung einer Silikonschlaufe die intraorale Bolusapplikation und Druckmessung erlaubte. Extraoral wurde das Mundstück einerseits an einer mit Flüssigkeit gefüllten Spritze zur Bolusapplikation und andererseits an ein digitales Manometer (GDUSB 1000®, Greisinger electronics, Regenstauf Deutschland) angeschlossen. Das genutzte Messgerät besaß die Fähigkeit, mit einer Frequenz von $1 \mathrm{kHz}$ Messungen in einem Bereich von 2000 bis -1000 mbar durchzuführen. Zum Schutz der Probanden und zur Sicherung der Messung wurde am Schlauchsystem ein Bakterienfilter und ein Wasserabscheider angebracht.

Mit dieser Versuchsanordnung wurden drei Schluckmodalitäten untersucht. Jede Modalität beinhaltete 10 Schluckvorgänge. Die erste Untersuchung bezog sich auf die aktive Einnahme eines Bolus aus Wasser. Die Probanden führten Saugimpulse aus, indem sie Flüssigkeit aus einer Spritze zogen und diese anschließend schluckten. Die zweite und dritte Untersuchung basierte auf der passiven Gabe eines Bolus, der in einem Volumen von $2 \mathrm{ml}$ appliziert wurde. In diesem Versuch wurde jeweils ein 2-ml-Bolus aus Wasser und aus Gel verabreicht.

Bei den erhobenen Daten konnten hauptsächlich negative Druckamplituden beobachtet werden. Dabei wurde ein Mittelwert von -290 mbar bei der aktiven Bolus-Einnahme, -31 mbar während der passiven Gabe eines Bolus aus Wasser und -37 mbar bei der passiven Gabe eines Bolus aus Gel gemessen. Auch die Dauer der Schluckereignisse wurde gemessen. Hierbei ergab sich ein Mittelwert von 5.1 s bei der aktiven Bolus- 
Einnahme, $1.8 \mathrm{~s}$ bei der passiven Gabe eines Bolus aus Wasser und $1.5 \mathrm{~s}$ bei der passiven Gabe eines Bolus aus Gel.

In Abhängigkeit von der Art der Bolusapplikation und der Boluskonsistenz konnten signifikante Differenzen zwischen den erhobenen Druckamplituden und Druckverläufen beobachtet werden. Die aktive Einnahme eines Bolus zeigte höhere negative Druckverläufe sowie eine längere Dauer als bei der passiven Gabe eines Bolus. Auch signifikante Unterschiede zwischen den Konsistenzen konnten beobachtet werden: die Gabe eines Bolus aus Gel wies polyphasische Kurven auf. Im Vergleich dazu zeigten sich vorwiegend monophasische Kurven beim Schlucken von Wasser.

Der vorgeschlagene Schlucktest zeigte eine einfache Anwendbarkeit und konnte bei allen Probanden problemlos durchgeführt werden. Der technische Aufwand war gering und die Untersuchung brachte keine Nebenwirkungen für die Probanden mit sich.

Anhand dieses Tests wurden Datensätze zur Schluckfunktion erzeugt, welche qualitativ und quantitativ ausgewertet wurden und als Normwert für zukünftige Untersuchungen dienen. Die Interpretation der erhobenen Daten anhand des biofunktionellen Modells ermöglicht eine methodische Erfassung der Schluckphysiologie. Eine klinische Anwendung bietet sich als diagnostischer Test sowie auch in der Übungstherapie an.

Die durchgeführten Untersuchungen konnten die funktionelle Ähnlichkeit der Funktionen Saugen und Schlucken belegen und zeigten, dass das vom biofunktionellen Modell ausgewiesene Kompartiment 2 offensichtlich zum Transport von Flüssigkeiten einen Saugmechanismus und nicht einen Propulsionsmechanismus durch Verdrängen des Bolus bevorzugt. 


\section{Anhang}

\subsection{Abkürzungsverzeichnis}

\begin{tabular}{|c|c|}
\hline $\mathrm{ABE}$ & Aktive Bolus-Einnahme \\
\hline $\mathrm{BFE}$ & Biofunktionelle Einheiten \\
\hline BFK & Biofunktionelles Kompartiment \\
\hline $\mathrm{cm}$ & Zentimeter \\
\hline EMA & Elektromagnetische Artikulographie \\
\hline $\mathrm{HNO}$ & Hals-Nasen-Ohren-Heilkunde \\
\hline IOPI & Iowa Performance Instrument \\
\hline $\mathrm{kHz}$ & Kilohertz \\
\hline $\mathrm{m}$ & Männlich \\
\hline M. & Musculus \\
\hline mbar & Millibar \\
\hline $\mathrm{Mm}$ & Musculi \\
\hline MRT & Magnetresonanztomographie \\
\hline MW & Mittelwert \\
\hline N. & Nervus \\
\hline $\mathrm{p}$ & $\mathrm{p}$-Wert \\
\hline PGG & Passive Gel-Bolus-Gabe \\
\hline PVC & Polyvinylchlorid \\
\hline PWG & Passive Wasser-Bolus-Gabe \\
\hline
\end{tabular}


r

S

SD

SE

V

W

z.B.

ZRM
r-Wert

Sekunde

Standardabweichung

Standardfehler

Verschlüsse

Weiblich

Zum Beispiel

Zungenrepositionsmanöver 


\subsection{Abbildungsverzeichnis}

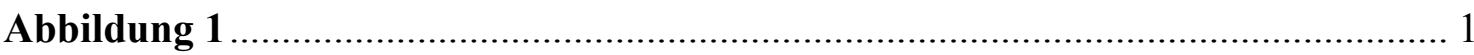

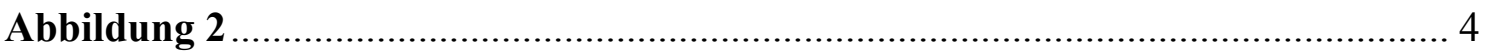

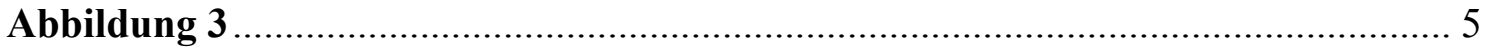

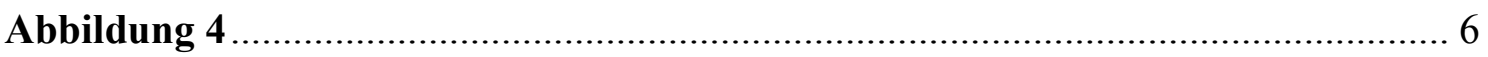

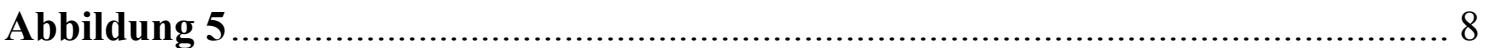

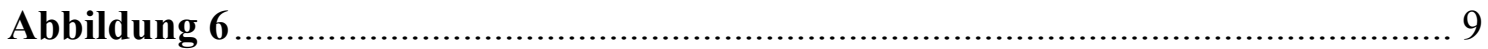

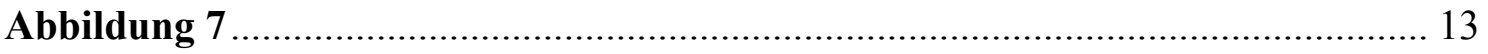

Abbildung 8

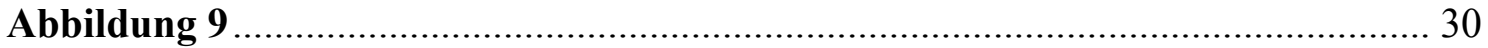

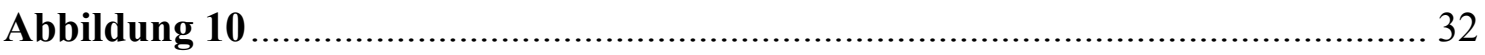

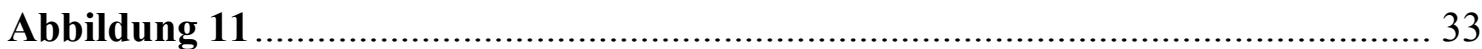

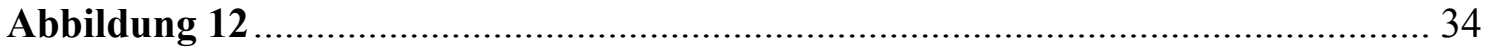

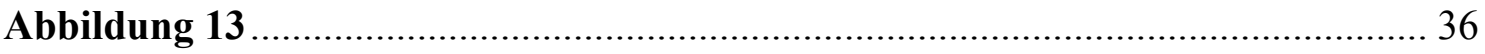

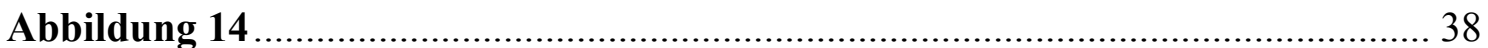

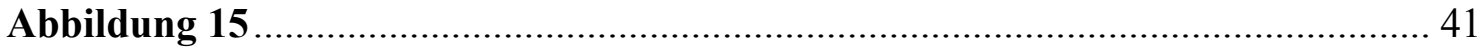

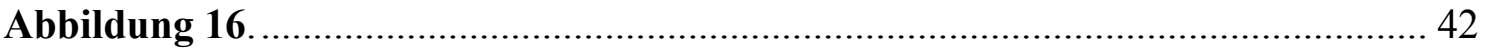

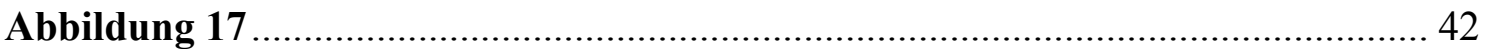

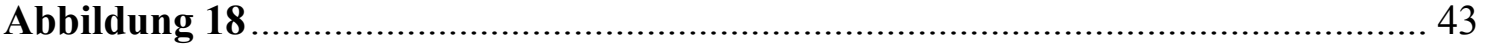

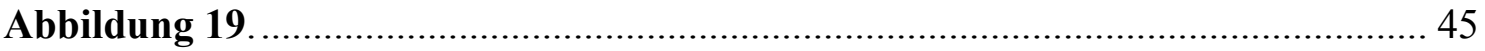

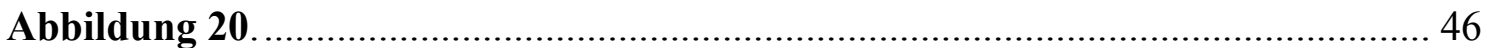

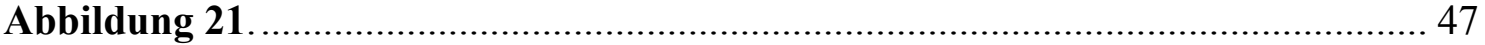




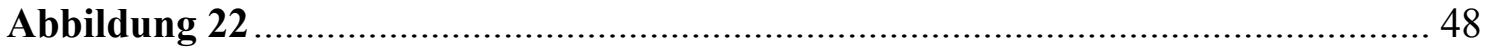

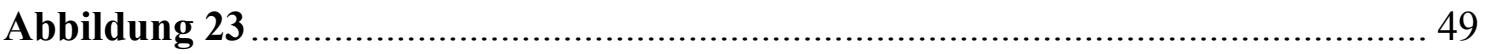

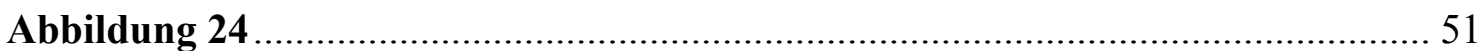

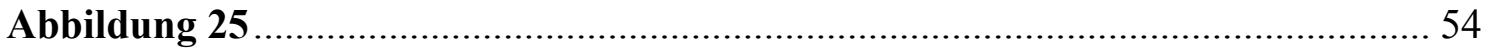

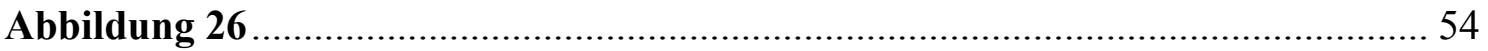

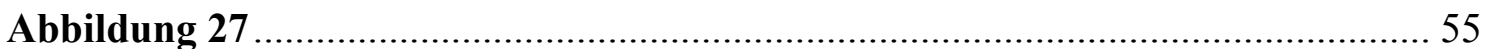

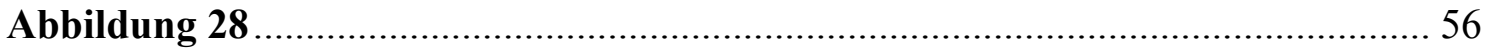

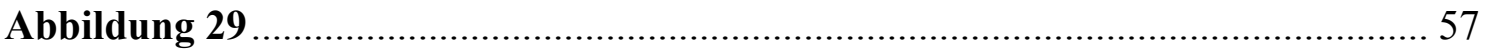

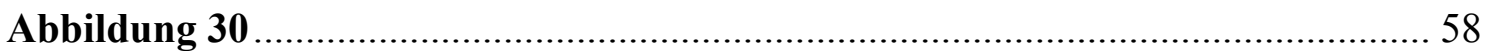

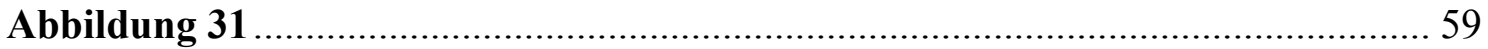

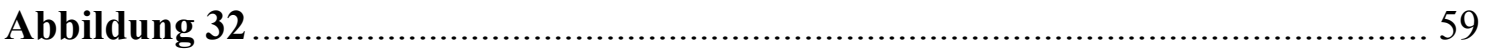

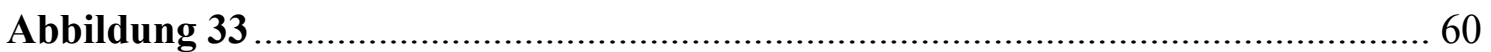

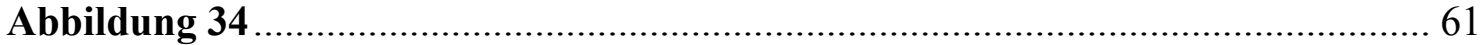

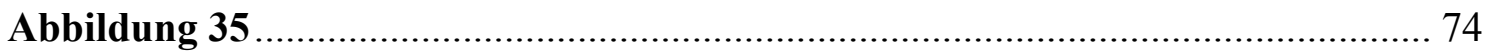




\subsection{Tabellenverzeichnis}

Tabelle 1

Tabelle 2

Tabelle 3

Tabelle 4

Tabelle 5

Tabelle 6

Tabelle 7 58

Tabelle 8 60 


\subsection{Datentabelle der Ergebnisse}

Amplituden und Dauer bei der gesamten Untersuchung bei allen Probanden (MW = Mittelwert, SD = Standardabweichung).

\begin{tabular}{|c|c|c|c|c|c|}
\hline Proband & Modalität & $\begin{array}{c}\text { MW } \\
\text { Amplitude }\end{array}$ & $\begin{array}{c}\text { SD } \\
\text { Amplitude }\end{array}$ & $\begin{array}{l}\text { MW } \\
\text { Dauer }\end{array}$ & $\begin{array}{l}\text { SD } \\
\text { Dauer }\end{array}$ \\
\hline \multirow[t]{3}{*}{1} & $\mathrm{ABE}$ & 287.3000 & 46.2530 & 5.650300 & 1.196565 \\
\hline & PWG & 31.7000 & 15.6706 & 5.256300 & 3.218566 \\
\hline & PGG & 67.6000 & 50.2819 & 3.239800 & 1.859435 \\
\hline \multirow[t]{3}{*}{2} & $\mathrm{ABE}$ & 376.2000 & 52.9545 & 4.525100 & 1.239039 \\
\hline & PWG & 41.6000 & 16.5677 & 2.949000 & 0.824347 \\
\hline & PGG & 85.0000 & 44.0202 & 1.126400 & 0.640064 \\
\hline \multirow[t]{3}{*}{3} & $\mathrm{ABE}$ & 274.4000 & 23.5947 & 3.973000 & 0.259466 \\
\hline & PWG & 38.6000 & 15.7353 & 2.396000 & 0.935602 \\
\hline & PGG & 31.3000 & 17.3849 & 2.208200 & 1.056706 \\
\hline \multirow[t]{3}{*}{4} & $\mathrm{ABE}$ & 182.3000 & 18.1233 & 4.107900 & 0.672037 \\
\hline & PWG & 22.5000 & 16.5613 & 2.430200 & 1.857629 \\
\hline & PGG & 23.0000 & 10.6458 & 5.102800 & 2.622470 \\
\hline \multirow[t]{3}{*}{5} & $\mathrm{ABE}$ & 123.8000 & 19.7191 & 5.901900 & 1.259777 \\
\hline & PWG & 104.5000 & 22.1973 & 6.018600 & 1.270848 \\
\hline & PGG & 74.5000 & 18.1246 & 2.562600 & 0.948809 \\
\hline \multirow[t]{3}{*}{6} & $\mathrm{ABE}$ & 352.0000 & 78.1807 & 4.059550 & 0.890323 \\
\hline & PWG & 25.4000 & 55.1567 & 0.494000 & 0.371469 \\
\hline & PGG & 7.6000 & 6.9314 & 1.743100 & 1.645510 \\
\hline \multirow[t]{3}{*}{7} & $\mathrm{ABE}$ & 290.2000 & 20.3786 & 5.303300 & 0.820918 \\
\hline & PWG & 36.0000 & 35.7522 & 0.865800 & 0.549691 \\
\hline & PGG & 48.2000 & 16.6386 & 4.194300 & 1.033267 \\
\hline \multirow[t]{3}{*}{8} & $\mathrm{ABE}$ & 258.6000 & 19.5857 & 4.294600 & 0.480347 \\
\hline & PWG & 40.4000 & 32.5173 & 2.357200 & 1.059683 \\
\hline & $\begin{array}{l}\text { PGG } \\
\end{array}$ & 16.6000 & 5.9852 & 1.778500 & 1.299865 \\
\hline \multirow[t]{3}{*}{9} & $\mathrm{ABE}$ & 327.3000 & 47.1971 & 4.824100 & 0.746994 \\
\hline & PWG & 58.0000 & 53.8908 & 1.524300 & 0.856927 \\
\hline & PGG & 88.2000 & 55.1398 & 1.090000 & 0.578113 \\
\hline \multirow[t]{3}{*}{10} & $\mathrm{ABE}$ & 365.8000 & 69.6958 & 4.042400 & 0.755439 \\
\hline & PWG & 80.4000 & 21.5675 & 3.883500 & 0.880186 \\
\hline & $\begin{array}{l}\text { PGG } \\
\end{array}$ & 107.5000 & 27.5288 & 1.918000 & 1.008146 \\
\hline \multirow[t]{3}{*}{11} & $\mathrm{ABE}$ & 375.3000 & 34.5802 & 4.839600 & 1.038525 \\
\hline & PWG & 49.9000 & 25.3221 & 3.096700 & 1.975857 \\
\hline & PGG & 29.0000 & 12.3558 & 4.434300 & 3.382884 \\
\hline \multirow[t]{3}{*}{12} & $\mathrm{ABE}$ & 222.0000 & 44.7064 & 7.480500 & 1.770556 \\
\hline & PWG & 60.0000 & 23.5561 & 1.732000 & 0.789321 \\
\hline & PGG & 14.0000 & 8.1240 & 1.424200 & 0.864034 \\
\hline
\end{tabular}




\begin{tabular}{|c|c|c|c|c|c|}
\hline \multirow[t]{3}{*}{13} & $\mathrm{ABE}$ & 229.4000 & 24.6991 & 3.069500 & 0.897755 \\
\hline & PWG & 18.9000 & 10.8878 & 3.352900 & 1.965726 \\
\hline & PGG & 33.9000 & 14.9700 & 1.025300 & 0.567146 \\
\hline \multirow[t]{3}{*}{14} & $\mathrm{ABE}$ & 272.9000 & 90.9780 & 4.571200 & 0.448841 \\
\hline & PWG & 53.1000 & 26.9545 & 3.446800 & 2.742985 \\
\hline & PGG & 102.6000 & 63.6923 & 1.388800 & 0.823810 \\
\hline \multirow[t]{3}{*}{15} & $\mathrm{ABE}$ & 283.4000 & 21.2300 & 2.520000 & 0.292062 \\
\hline & PWG & 4.1000 & 16.1758 & 1.213400 & 1.555034 \\
\hline & PGG & 17.6000 & 13.2682 & 2.030400 & 1.814437 \\
\hline \multirow[t]{3}{*}{16} & $\mathrm{ABE}$ & 326.0000 & 12.9529 & 6.456900 & 1.016168 \\
\hline & PWG & 3.7000 & 5.3344 & 0.53100 & 0.362250 \\
\hline & PGG & 6.4000 & 11.9089 & 0.476300 & 0.424992 \\
\hline \multirow[t]{3}{*}{17} & $\mathrm{ABE}$ & 178.9000 & 36.3485 & 7.593300 & 2.299516 \\
\hline & PWG & 18.2000 & 11.1136 & 2.969300 & 1.397790 \\
\hline & PGG & 12.9000 & 8.4123 & 2.072400 & 1.170308 \\
\hline \multirow[t]{3}{*}{18} & $\mathrm{ABE}$ & 344.3000 & 25.3949 & 5.397500 & 1.725730 \\
\hline & PWG & 74.2000 & 32.9404 & 3.156800 & 1.003510 \\
\hline & PGG & 51.3000 & 25.0956 & 1.547300 & 1.054962 \\
\hline \multirow[t]{3}{*}{19} & $\mathrm{ABE}$ & 233.8000 & 23.5599 & 4.375300 & 1.412721 \\
\hline & PWG & 6.8000 & 8.2435 & 0.407900 & 0.234410 \\
\hline & PGG & 7.8000 & 5.6921 & 0.672900 & 0.264670 \\
\hline \multirow[t]{3}{*}{20} & $\mathrm{ABE}$ & 262.2000 & 56.8620 & 2.760800 & 0.421217 \\
\hline & PWG & 17.3000 & 20.6616 & 0.726600 & 0.660632 \\
\hline & PGG & -2.7000 & 8.2738 & 0.944200 & 0.445662 \\
\hline \multirow[t]{3}{*}{21} & $\mathrm{ABE}$ & 207.9000 & 20.1519 & 3.508800 & 0.670930 \\
\hline & PWG & 36.7000 & 15.6918 & 1.151100 & 0.635875 \\
\hline & PGG & 73.5000 & 22.9891 & 1.077100 & 0.372544 \\
\hline \multirow[t]{3}{*}{22} & $\mathrm{ABE}$ & 296.5000 & 27.8737 & 5.404400 & 1.579873 \\
\hline & PWG & 16.8000 & 14.2423 & 0.582200 & 0.289082 \\
\hline & PGG & 25.0000 & 10.7290 & 0.912800 & 0.740833 \\
\hline \multirow[t]{3}{*}{23} & $\mathrm{ABE}$ & 339.6000 & 31.5531 & 5.916900 & 1.111494 \\
\hline & PWG & 37.7000 & 16.8790 & 2.158800 & 1.487900 \\
\hline & PGG & 43.5000 & 55.5283 & 0.832400 & 0.504029 \\
\hline \multirow[t]{3}{*}{24} & $\mathrm{ABE}$ & 338.8000 & 28.7356 & 5.102000 & 0.882619 \\
\hline & PWG & 19.6000 & 5.1467 & 1.724300 & 0.935736 \\
\hline & PGG & 30.5000 & 17.3413 & 1.010700 & 0.456208 \\
\hline \multirow[t]{3}{*}{25} & $\mathrm{ABE}$ & 257.4000 & 33.0629 & 6.001700 & 0.283691 \\
\hline & PWG & 34.3000 & 23.7302 & 1.856900 & 0.432353 \\
\hline & PGG & 41.8000 & 31.5869 & 0.792100 & 0.471568 \\
\hline \multirow[t]{3}{*}{26} & $\mathrm{ABE}$ & 333.6000 & 23.2293 & 6.366100 & 1.040456 \\
\hline & PWG & 58.3000 & 36.2309 & 3.029000 & 1.267189 \\
\hline & PGG & 44.3000 & 34.2217 & 0.963600 & 0.710248 \\
\hline \multirow[t]{3}{*}{27} & $\mathrm{ABE}$ & 489.2000 & 41.6355 & 5.539400 & 1.123 .833 \\
\hline & PWG & 6.0000 & 3.7712 & 0.956700 & 0.453695 \\
\hline & PGG & 7.4000 & 4.9486 & 0.667900 & 0.363109 \\
\hline
\end{tabular}




\begin{tabular}{|c|c|c|c|c|c|}
\hline \multirow[t]{3}{*}{28} & $\mathrm{ABE}$ & 271.2000 & 26.8610 & 6.078900 & 1.309530 \\
\hline & PWG & 16.4000 & 10.8853 & 2.176200 & 1.006397 \\
\hline & PGG & 21.2000 & 18.3775 & 1.358400 & 0.950562 \\
\hline \multirow[t]{3}{*}{29} & $\mathrm{ABE}$ & 298.3000 & 14.0558 & 3.458100 & 0.539207 \\
\hline & PWG & 12.4000 & 9.5824 & 1.688000 & 1.788484 \\
\hline & PGG & 33.8000 & 27.8679 & 0.789200 & 0.586103 \\
\hline \multirow[t]{3}{*}{30} & $\mathrm{ABE}$ & 289.3000 & 73.3546 & 8.124800 & 1.796144 \\
\hline & PWG & 77.9000 & 30.8741 & 2.730100 & 0.920351 \\
\hline & PGG & 32.8000 & 16.5382 & 1.632000 & 0.613179 \\
\hline \multirow[t]{3}{*}{31} & $\mathrm{ABE}$ & 335.4000 & 50.3746 & 9.294200 & 1.129983 \\
\hline & PWG & 22.9000 & 22.3231 & 2.099700 & 1.150688 \\
\hline & PGG & 11.7000 & 8.3140 & 0.865600 & 0.546834 \\
\hline \multirow[t]{3}{*}{32} & $\mathrm{ABE}$ & 273.5000 & 23.5337 & 3.977100 & 0.511818 \\
\hline & PWG & 9.1000 & 13.5438 & 0.822300 & 0.780997 \\
\hline & PGG & 22.0000 & 24.6667 & 1.443200 & 1.036932 \\
\hline \multirow[t]{3}{*}{33} & $\mathrm{ABE}$ & 325.1000 & 39.8231 & 5.566400 & 0.878646 \\
\hline & PWG & 53.2000 & 31.9993 & 0.967400 & 0.457864 \\
\hline & PGG & 21.4000 & 19.7720 & 0.763700 & 0.309980 \\
\hline \multirow[t]{3}{*}{34} & $\mathrm{ABE}$ & 379.0000 & 43.3846 & 8.419100 & 1.483931 \\
\hline & PWG & 6.2000 & 4.2895 & 0.821500 & 0.734029 \\
\hline & PGG & 71.4000 & 16.6946 & 1.545200 & 0.739568 \\
\hline \multirow[t]{3}{*}{35} & $\mathrm{ABE}$ & 477.1000 & 41.3936 & 5.368000 & 1.268307 \\
\hline & PWG & 11.9000 & 5.4863 & 1.334700 & 0.755954 \\
\hline & PGG & 52.2000 & 24.9123 & 0.818500 & 0.363524 \\
\hline \multirow[t]{3}{*}{36} & $\mathrm{ABE}$ & 266.6000 & 16.2631 & 5.382800 & 1.540965 \\
\hline & PWG & 6.0000 & 9.5801 & 1.159700 & 1.629408 \\
\hline & PGG & 15.3000 & 4.8086 & 0.728800 & 0.254504 \\
\hline \multirow[t]{3}{*}{37} & $\mathrm{ABE}$ & 566.7000 & 34.3804 & 5.287900 & 0.743766 \\
\hline & PWG & 36.0000 & 21.6179 & 1.349300 & 0.337893 \\
\hline & PGG & 35.7000 & 24.8643 & 0.984800 & 0.375145 \\
\hline \multirow[t]{3}{*}{38} & $\mathrm{ABE}$ & 232.4000 & 25.5873 & 2.723200 & 0.837235 \\
\hline & PWG & 45.3000 & 21.0663 & 1.079600 & 0.424249 \\
\hline & PGG & 23.4000 & 12.5096 & 0.788800 & 0.355739 \\
\hline \multirow[t]{3}{*}{39} & $\mathrm{ABE}$ & 236.5000 & 23.7077 & 2.553300 & 0.211363 \\
\hline & PWG & 72.7000 & 37.6004 & 1.710800 & 0.477236 \\
\hline & PGG & 108.4000 & 32.6129 & 1.434800 & 0.623397 \\
\hline \multirow[t]{3}{*}{40} & $\mathrm{ABE}$ & 478.3000 & 23.6739 & 5.399700 & 0.537377 \\
\hline & PWG & 8.3000 & 4.2439 & 1.356900 & 0.872809 \\
\hline & PGG & 65.7000 & 25.1884 & 1.860000 & 0.800815 \\
\hline \multirow[t]{3}{*}{41} & $\mathrm{ABE}$ & 163.6000 & 20.1174 & 3.498600 & 1.289653 \\
\hline & PWG & 28.1000 & 14.1771 & 2.912600 & 1.936471 \\
\hline & PGG & 23.5000 & 9.8008 & 2.713800 & 1.303471 \\
\hline \multirow[t]{3}{*}{42} & $\mathrm{ABE}$ & 319.0000 & 47.4763 & 4.273600 & 0.294740 \\
\hline & PWG & 19.6000 & 15.6645 & 0.859100 & 0.322514 \\
\hline & PGG & 29.8000 & 20.3404 & 1.002000 & 0.383159 \\
\hline
\end{tabular}




\begin{tabular}{|c|c|c|c|c|c|}
\hline \multirow[t]{3}{*}{43} & $\mathrm{ABE}$ & 124.7000 & 38.5373 & 2.979600 & 0.735236 \\
\hline & PWG & 17.1000 & 15.0956 & 1.029400 & 0.608334 \\
\hline & PGG & 12.2000 & 4.0497 & 1.305900 & 0.830383 \\
\hline \multirow[t]{3}{*}{44} & $\mathrm{ABE}$ & 233.9000 & 20.0192 & 5.772700 & 1.353250 \\
\hline & PWG & 19.8000 & 10.0642 & 1.183600 & 0.636110 \\
\hline & PGG & 38.3000 & 32.4792 & 1.971300 & 0.651734 \\
\hline \multirow[t]{3}{*}{45} & $\mathrm{ABE}$ & 326.5000 & 56.5455 & 5.453100 & 1.775768 \\
\hline & PWG & 17.2000 & 8.3639 & 1.106500 & 0.725718 \\
\hline & PGG & 26.5000 & 10.4907 & 2.544100 & 1.357255 \\
\hline \multirow[t]{3}{*}{46} & $\mathrm{ABE}$ & 239.5000 & 32.3840 & 9.668200 & 2.398615 \\
\hline & PWG & 6.9000 & 2.3781 & 0.522680 & 0.189873 \\
\hline & PGG & 43.9000 & 48.9386 & 0.542300 & 0.236394 \\
\hline \multirow[t]{3}{*}{47} & $\mathrm{ABE}$ & 412.8000 & 40.2652 & 4.894700 & 0.393893 \\
\hline & PWG & 63.9000 & 20.8936 & 3.324000 & 0.706385 \\
\hline & PGG & 94.3000 & 32.5203 & 3.257200 & 0.331733 \\
\hline \multirow[t]{3}{*}{48} & $\mathrm{ABE}$ & 247.1000 & 35.0347 & 5.555500 & 0.520562 \\
\hline & PWG & 14.0000 & 16.4520 & 0.697600 & 0.404124 \\
\hline & PGG & 8.6000 & 5.8916 & 0.687300 & 0.413284 \\
\hline \multirow[t]{3}{*}{49} & $\mathrm{ABE}$ & 98.0000 & 78.5734 & 4.535400 & 0.633005 \\
\hline & PWG & 17.5000 & 21.9254 & 0.656400 & 0.640444 \\
\hline & PGG & 6.7000 & 8.6288 & 0.300900 & 0.224773 \\
\hline \multirow[t]{3}{*}{50} & $\mathrm{ABE}$ & 180.3000 & 30.5616 & 8.336800 & 2.486288 \\
\hline & PWG & 30.3000 & 21.2239 & 1.826300 & 0.673234 \\
\hline & PGG & 8.1000 & 4.2019 & 1.217800 & 0.535203 \\
\hline \multirow[t]{3}{*}{51} & $\mathrm{ABE}$ & 245.4000 & 7.8768 & 2.216400 & 0.376608 \\
\hline & PWG & 7.1000 & 3.2128 & 0.500200 & 0.250456 \\
\hline & PGG & 4.8000 & 12.0167 & 0.424000 & 0.361860 \\
\hline \multirow[t]{3}{*}{52} & $\mathrm{ABE}$ & 248.9000 & 42.1623 & 4.376800 & 0.874251 \\
\hline & PWG & 34.6000 & 24.0102 & 2.394500 & 0.750511 \\
\hline & PGG & 27.0000 & 20.1439 & 1.808500 & 1.587393 \\
\hline
\end{tabular}




\section{Literaturverzeichnis}

\section{Ardran G, Kemp F (1951):}

The mechanism of swallowing.

Proc R Soc Med 44, 1038-1040

Ardran G, Kemp F, Lind J (1958):

A cineradiographic study of bottle feeding.

Br J Radiol 31, 11-22

\section{Atkinson M, Kramer P, Wyman SM, Ingelfinger FJ (1957):}

The dynamics of swallowing. Normal pharyngeal mechanisms.

J Clin Invest $\underline{36}, 581-588$

Aviv JE (2000):

Prospective, randomized outcome study of endoscopy versus modified barium swallow in patients with dysphagia.

Laryngoscope $\underline{110}, 563-574$

\section{Bartolome G, Schröter-Morasch H:}

Schluckstörungen: Diagnostik und Rehabilitation.

4. Auflage; Urban \& Fischer Verlag, München 2010

\section{Blonski W, Vela M, Hila A, Castell D (2008):}

Normal values for manometry performed with swallows of viscous test material.

Scand J Gastroenterol $\underline{43}, 155-160$

Blot WJ, McLaughlin JK, Winn DM, Austin DF, Greenberg RS, Preston-Martin S, Bernstein L, Schoenberg JB, Stemhagen A, Fraumeni JF Jr . (1988):

Smoking and drinking in relation to oral and pharyngeal cancer.

Cancer Res $\underline{48}, 3282-3287$

\section{Böcker R, Wein B, Klajman S (1989):}

Ultrasound sonography of active and passive tongue motility.

Folia Phoniatr (Basel) $\underline{41}$, 277-282 


\section{Böhme G, Hrsg.:}

Sprach-, Sprech-, Stimm- und Schluckstörungen, Band 1.

4. Auflage; Urban \& Fischer Verlag, München 2003

\section{Bootz F:}

HNO- Erkrankungen in der Pädiatrie.

Wissenschaftliche Verlagsgessellschaft, Stuttgart 1995

\section{Brawley R, Sedwick J (1938):}

Gnathodynamometer.

Am J Orthod 24, 256-258

Breyer T, Echternacht M, Arndt S, Richter B, Speck O, Schumacher M, MarkI M (2009):

Dynamic magnetic resonance imaging of swallowing and laryngeal motion using parallel imaging at $3 \mathrm{~T}$.

Magn Reson Imaging 27, 48-54

\section{Chi-Fishman G (2005):}

Quantitative lingual, pharyngeal and laryngeal ultrasonography in swallowing research: a technical review.

Clin Linguist Phon $\underline{19}$, 589-604

\section{Chi-Fishman G, Stone M, McCall G (1998):}

Lingual action in normal sequential swallowing.

J Speech Lang Hear Res $\underline{41}, 771-785$

\section{Choi B, Kleinheinz J, Joos U, Komposch G (1991):}

Sucking efficiency of early orthopaedic plate and teats in infants with cleft lip and palate.

Int J Oral Maxillofac Surg 20, 167-169

\section{Christensen J (1989):}

Developmental approach to pediatric neurogenic dysphagia.

Dysphagia $\underline{3}, 131-134$ 
Cichero JAY, Murdoch BE (2002):

Acoustic signature of the normal swallow: characterization by age, gender, and bolus volume.

Ann Otol Rhinol Laryngol 111, 623-632

\section{Clark H, Solomon N (2011):}

Age and sex differences in orofacial strength.

Dysphagia 27, 2-9

\section{Cook IJ (2009):}

Oropharyngeal dysphagia.

Gastroenterol Clin North Am $\underline{38}, 411-431$

Cook IJ, Dodds WJ, Dantas RO, Kern MK, Massey BT, Shaker R, Hogan WJ (1989):

Timing of videofluoroscopic, manometric events, and bolus transit during the oral and pharyngeal phases of swallowing.

Dysphagia $\underline{4}, 8-15$

Cooper-Brown L, Copeland S, Dailey S, Downey D, Petersen MC, Stimson C, Van Dyke DC (2008):

Feeding and swallowing dysfunction in genetic syndromes.

Dev Disabil Res Rev 14, 147-157

Dantas R, Dodds W, Massey B, Shaker R, Cook I (1990):

Manometric characteristics of glossopalatal sphincter.

Dig Dis Sci $\underline{35}$, 161-166

Dauber W, Feneis, H:

Bild-Lexikon der Anatomie.

9. Auflage; Georg Thieme Verlag, Stuttgart 2005

Dictor M, Johnson A (2000):

Association of inverted sinonasal papilloma with non-sinonasal head and neck carcinoma.

Int J Cancer $\underline{85}$, 811-814 


\section{Dodds WJ (1989):}

Physiology of swallowing.

Dysphagia $\underline{3}, 171-178$

Dodds WJ, Taylor AJ, Stewart ET, Kern MK, Logemann JA, Cook IJ (1989):

Tipper and dipper types of oral swallows.

AJR Am J Roentgenol 153, 1197-1199

\section{Dodds WJ, Stewart ET, Logemann JA (1990):}

Physiology and radiology of the normal oral and pharyngeal phases of swallowing.

AJR Am J Roentgenol 154, 953-963

\section{EI Serag HB, Hepworth EJ, Lee P, Sonnenberg A (2001):}

Gastroesophageal reflux disease is a risk factor for laryngeal and pharyngeal cancer.

Am J Gastroenterol 96, 2013-2018

\section{Engelke, W:}

Systematische Rhonchopathiebehandlung in der zahnärztlichen Praxis.

Cuvillier Verlag, Göttingen 2007

\section{Engelke W, Engelke D, Schwestka R (1990):}

Clinical and instrumental examination of the tongue motor function.

Dtsch Zahnarztl Z 노, 11-16

\section{Engelke W, Bruns T, Striebeck M, Hoch G (1996):}

Midsagittal velar kinematics during production of VCV sequences.

Cleft Palate Craniofac J $\underline{33}$, 236-244

\section{Engelke W, Mendoza M, Repetto G (2006):}

Preliminary radiographic observations of the tongue-repositioning manouvre.

Eur J Orthod 28, 618-623 
Engelke W, Engelhardt W, Mendoza-Gärtner M, Deccó O, Barriero J, Knösel M (2010):

Functional treatment of snoring based on the tongue-repositioning manouvre.

Eur J Orthod $\underline{32}, 490-495$

\section{Engelke W, Jung K, Knösel M (2011):}

Intra-oral compartment pressures: a biofunctional model and experimental measurements under different conditions of posture.

Clin Oral Investig 15(2), 165-176

Feinberg M, Knebl J, Tully J, Segall L (1990):

Aspiration and the elderly.

Dysphagia $\underline{5}, 61-71$

Felton S, Gaige T, Reese T, Wedeen V, Gilbert R (2007):

Mechanical basis for lingual deformation during the propulsive phase of swallowing as determined by phase contrast magnetic resonance imaging.

J Appl Physiol 103, 255-265

Ferlay J, Shin H, Bray F, Forman D, Mathers C, Parkin D (2010):

Globocan 2008 v1.2, Cancer incidence and mortality worldwide: IARC Cancerbase No. 10 [internet] Lyon, France: International Agency for Research on Cancer; http://globocan.iarc.fr,accessed on 03/04/2011

Field L, Weiss C (1989):

Dysphagia with head injury.

Brain Inj $\underline{3}, 19-26$

\section{Foucart J, Carpentier P, Paioni D, Rabischong P, Pharaboz C (1998):}

Kinetic magnetic resonance imaging analysis of swallowing: a new approach to pharyngeal function.

Surg Radiol Anat 20: 53-55

Friedrich G, Bigenzahn W (1995):

Phoniatrie.

Hans Huber Verlag, Bern 1995 


\section{Fröhlich K, Thüer U, Ingervall B (1991):}

Pressure from the tongue on the teeth in young adults.

Angle Orthod 61, 17-24

\section{Fröhlich K, Ingervall B, Thüer U (1992):}

Further studies of the pressure from the tongue on the teeth in young adults.

Eur J Orthod 14, 229-239

\section{Gaziano JE (2002):}

Evaluation and management of oropharyngeal dysphagia in head and neck cancer.

Cancer Control $\underline{9}, 400-409$

Geddes DT, Kent JC, Mitoulas LR, Hartmann PE (2008):

Tongue movement and intra-oral vacuum in breastfeeding infants.

Early Hum Dev $\underline{84}, 471-477$

\section{German R, Crompton A, Levitch L, Thexton A (1992):}

The mechanism of suckling in two species of infant mammal: miniature pigs and longtailed macaques.

J Exp Zool 261, 322-330

\section{Gleeson D (1999):}

Oropharyngeal swallowing and aging: a review.

J Commun Disord 32, 373-395

\section{Gould M, Picton D (1964):}

Study of pressures exerted by the lips and cheeks on the teeth of subjects with normal occlusion.

Arch Oral Biol $\underline{9}, 469-472$

\section{Graber T (1963):}

The "three Ms": muscles, malformation, malocclusion.

Am J Orthod $\underline{49}, 418-450$ 
Gross AM, Kellum GD, Halle ST, Messer SC, Benson BA, Sisakun SL, Bishop FW (1990):

Myofunctional and dentofacial relationships in second grade children.

Angle Orthod $\underline{60}, 247-253$

Habel A, Sell D, Mars M (1996):

Management of cleft lip and palate.

Arch Dis Child $\underline{74}$, 360-366

Hamdy S, Rothwell JC, Brooks DJ, Bailey D, Aziz Q, Thompson DG (1999):

Identification of the cerebral loci processing human swallowing with H2(15)O PET activation.

J Neurophysiol $\underline{81}, 1917-1926$

\section{Hardcastle WJ, Gibbon FE, Jones W (1991):}

Visual display of tongue-palate contact: electropalatography in the assessment and remediation of speech disorders.

Br J Disord Commun 26, 41-74

\section{Hendrix T (1993):}

Coordination of peristalsis in pharynx and esophagus.

Dysphagia $\underline{8}, 74-78$

\section{Hesse S, Schnell G:}

Sensoren für die Prozess und Fabrikautomation Funktion - Ausführung - Anwendung.

3. Auflage; Vieweg \& Sohn Verlag, Wiesbaden 2004

Hiiemae KM, Palmer JB (2003):

Tongue movements in feeding and speech.

Crit Rev Oral Biol Med 14, 413-429

Hind JA, Nicosia MA, Gangnon R, Robbins J (2005):

The effects of intraoral pressure sensors on normal young and old swallowing patterns.

Dysphagia 20, 249-253 


\section{Hiss SG, Postma GN (2003):}

Fiberoptic endoscopic evaluation of swallowing.

Laryngoscope $\underline{113}, 1386-1393$

\section{Hiss SG, Strauss M, Treole K, Stuart A, Boutilier S (2004):}

Effects of age, gender, bolus, volume, bolus viscosity and gustation on swallowing apnea onset relative to lingual bolus propulsion onset in normal adults.

J Speech Lang Hear Res $\underline{47}, 572-583$

\section{Hoffman DM, Singh B, Thomas JH:}

Handbook of vacuum science and technology.

Academic Press Verlag, San Diego 1998

\section{Howell AR, Brudevold F (1950):}

Vertical forces used during chewing of food.

J Dent Res 29, 133-136

\section{Howell AR, Manly RS (1948):}

An Electronic strain gauge for measuring oral forces.

J Dent Res 27, 705-712

Iida S, Harada T, Okamoto M, Inada Y, Kogo M, Masuda Y (2003):

Soft palate movement during sucking behavior.

Dysphagia $\underline{18}, 96-100$

\section{Jäghagen EL, Bodin I, Isberg A (2008):}

Pharyngeal swallowing dysfunction following treatment for oral and pharyngeal cancer-association with diminished intraoral sensation and discrimination ability.

Head Neck $\underline{30}, 1344-1351$

\section{Jensen A, Garnier Y, Middelanis J, Berger R (2003):}

Perinatal brain damage--from pathophysiology to prevention.

Eur J Obstet Gynecol Reprod Biol 22, 70-79 


\section{Kawashima K, Motohashi Y, Fujishima J (2004):}

Prevalence of dysphagia among community-dwelling elderly individuals as estimated using a questionnaire for dysphagia screening.

Dysphagia 19(4), 266-271

\section{Kennedy D, Kieser J, Bolter C, Swain M, Singh B, Waddell J (2010):}

Tongue pressure patterns during water swallowing.

Dysphagia 25, 11-19

\section{Kahrilas P, Lin S, Logemann J, Ergun G, Facchini F (1993):}

Deglutitive tongue action: volume accommodation and bolus propulsion.

Gastroenterology 104, 152-162

\section{Kahrilas P, Lin S, Chen J, Logemann J (1996):}

Oropharyngeal accommodation to swallow volume.

Gastroenterology $\underline{111}, 297-306$

\section{Kahrilas P, Lin S, Rademaker A, Logemann J (1997):}

Impaired deglutitive airway protection: a videofluoroscopic analysis of severity and mechanism.

Gastroenterology $\underline{11}, 1457-1464$

Kieser J, Singh B, Swain M, Ichm I, Waddel J, Kennedy D, Foster K, Livingston V (2008):

Measuring intraoral pressure: adaptation of a dental appliance allows measurement during function.

Dysphagia $\underline{23}, 237-243$

\section{Kittel A:}

Myofunktionelle Therapie.

Schulz-Kirchner Verlag, Idstein 2001

Konaka K, Kondo J, Hirota N, Tamine K, Hori K, Ono T, Maeda Y, Sakoda S, Naritomi H (2010):

Relationship between tongue pressure and dysphagia in stroke patients.

Eur Neurol $\underline{64}, 101-107$ 


\section{Kuhlemeier K (1994):}

Epidemiology and dysphagia.

Dysphagia $\underline{9}, 209-217$

\section{Kydd W (1956):}

Quantitative analysis of forces of the tongue.

J Dent Res $\underline{35}, 171-174$

\section{Kydd W, Akamine J, Mendel R, Kraus B (1963):}

Tongue and lip forces exerted during deglutition in subjects with and without an anterior open bite.

J Dent Res $\underline{42}, 858-866$

\section{Lambrechts H, De Baets E, Fiezws S, Willems G (2010):}

Lip and tongue pressure in orthodontic patients.

Eur J Orthod $\underline{32}, 466-471$

\section{Lang I (2009):}

Brain stem control of the phases of swallowing.

Dysphagia 24, 333-348

\section{Langmore SE, Schatz K, Olson N (1991):}

Endoscopic and videofluoroscopic evaluations of swallowing and aspiration.

Ann Otol Rhinol Laryngol 100, 678-681

Langmore SE, Terpenning MS, Schork A, Chen Y, Murray JT, Lopatin D, Loesche WJ (1998):

Predictors of aspiration pneumonia: how important is dysphagia?

Dysphagia $\underline{13}, 69-81$

\section{Lavelle C, Hrsg.:}

Applied physiology of the mouth.

J Wright and Sons Verlag, Bristol 1957 


\section{Lazarus CL (1993):}

Effects of radiation therapy and voluntary maneuvers on swallow function in head and neck cancer patients.

Clin Commun Disord $\underline{3}, 11-20$

\section{Lazarus CL, Logemann JA (1987):}

Swallowing disorders in closed head trauma patients.

Arch Phys Med Rehabil $\underline{68}, 79-84$

Lazarus CL, Logemann JA, Rademaker AW, Kahrilas P, Pajak T, Lazar R, Halper A (1993):

Effects of bolus volume, viscosity and repeated swallows in nonstroke subjects and stroke patients.

Arch Phys Med Rehabil 4ㅡ, 1066-1070

Lazarus CL, Logemann JA, Pauloski BR, Rademaker AW, Larson CR, Mittal BB, Pierce M (2000):

Swallowing and tongue function following treatment for oral and oropharyngeal cancer.

J Speech Lang Hear Res $\underline{43}$, 1011-1023

\section{Lear C, Moorrees C (1966):}

Swallowing frequency: a detection system employing FM telemetry.

J Dent Res $\underline{45}, 1222$

\section{Lear C, Moorrees C (1969):}

Buccolingual muscle force and dental arch form.

Am J Orthod 56, 379-393

\section{Lippert H:}

Lehrbuch Anatomie.

7. Auflage; Urban \& Fischer Verlag, München 2006

\section{Logemann JA (1985):}

Aspiration in head and neck surgical patients.

Ann Otol Rhinol Laryngol 94, 373-376 


\section{Logemann JA (1988):}

Swallowing physiology and pathophysiology.

Otolaryngol Clin North Am 21, 613-623

\section{Logemann JA:}

Manual for the videofluorographic study of swallowing.

2. Auflage; PRO-ED Verlag, Austin-Texas 1993

\section{Logemann JA:}

Evaluation and treatment of swallowing disorders.

2. Auflage; PRO-ED Verlag, Austin-Texas 1998

\section{Logemann JA, Rademaker AW, Pauloski BR, Ohmae Y, Kahrilas PJ (1998):}

Normal swallowing physiology as viewed by videofluoroscopy and videoendoscopy.

Folia Phoniatr Logop 50, 311-319

\section{Love RJ, Hagerman EL, Taimi EG (1980):}

Speech performance, dysphagia and oral reflexes in cerebral palsy.

J Speech Hear Disord 45, 59-75

\section{Magendie F:}

Memoire sur L'usage de l'epiglotte dans la deglutition.

Meguignon-Marvis Verlag, Paris 1813

\section{Magendie F, Revere J:}

An elementary treatise on human physiology.

5. Auflage; Harper \& Brothers Verlag, New York 1855

\section{Marik P, Kaplan D (2003):}

Aspiration pneumonia and dysphagia in the elderly.

Chest $\underline{124}, 328-336$

Martin-Harris B, Brodsky M, Michel Y, Ford C, Walters B, Heffener J (2005):

Breathing and swallowing dynamics across the adult lifespan.

Arch Otolaryngol Head Neck Surg 131, 762-770 
Masarei A, Sell D, Habel A, Mars M, Sommerland B, Wade A (2007):

The nature of feeding in infants with unrepaired cleft lip and/or palate compared with healthy noncleft infants.

Cleft Palate Craniofac J $\underline{44}, 321-328$

\section{Mathew O, Clark M, Pronske M, Luna-Solarzano H, Peterson M (1985):}

Breathing pattern and ventilation during oral feeding in term newborn infants.

J Pediatr $\underline{106}, 810-813$

\section{Matsuo K, Palmer JB (2008):}

Anatomy and physiology of feeding and swallowing: normal and abnormal.

Phys Med Rehabil Clin N Am $\underline{19}$, 691-707

\section{Matsuo K, Palmer JB (2009):}

Coordination of mastication, swallowing and breathing.

Jpn Dent Sci Rev $\underline{45}, 31-40$

\section{McConnel FM, Cerenko D, Mendelsohn MS (1988a):}

Manofluorographic analysis of swallowing.

Otolaryngol Clin North Am 21, 625-635

\section{McConnel FM, Cerenko D, Jackson RT, Hersh T (1988b):}

Clinical application of the manofluorogram.

Laryngoscope $\underline{98}, 705-711$

McConnel FM, Logemann JA, Rademaker AW, Pauloski BR, Baker SR, Lewin J, Shedd D, Heiser MA, Cardinale S, Collins S (1994):

Surgical variables affecting postoperative swallowing efficiency in oral cancer patients: a pilot study.

Laryngoscope $\underline{104}, 87-90$

McLaughlin JK, Gridley G, Block G, Winn DM, Preston-Martin S, Schoenenberg JB, Greenberg RS, Stemhagen A, Austin DF, Ershow AG, Blot WJ, Fraumeni JF (1988):

Dietary factors in oral and pharyngeal cancer.

J Natl Cancer Inst $\underline{80}, 1238-1243$ 


\section{Melsen B, Attina L, Santuari M, Attina A (1987):}

Relationships between swallowing pattern, mode of respiration, and development of malocclusion.

Angle Orthod 28, 618-623

\section{Miller A (1982):}

Deglutition.

Physiol Rev $\underline{62}, 129-184$

\section{Miller J, Watkin K (1996):}

The influence of bolus volumen and viscosity on anterior lingual force during the oral stage of swallowing.

Dysphagia $\underline{11}, 117-124$

\section{Misurva K (1976):}

Functional anatomy of tensor palati and levator palati muscles.

Arch Otolaryngol 102, 265-270

Mittal BB, Pauloski BR, Haraf DJ, Pelzer HJ, Argiris A, Vokes EE, Rademaker A, Logemann JA (2003):

Swallowing dysfunction--preventative and rehabilitation strategies in patients with head-and-neck cancers treated with surgery, radiotherapy, and chemotherapy: a critical review.

Int J Radiat Oncol Biol Phys $\underline{57}$, 1219-1230

\section{Morinière S, Boiron M, Beutter P (2008):}

Swallowing sound signal: description in normal and laryngectomized subjects.

Ann Otolaryngol Chir Cervicofac 125, 1-10

\section{Morris I (1988):}

Functional anatomy of the upper airway.

Emerg Med Clin North Am $\underline{6}$, 639-339

\section{Motsch C (2005):}

Impaired swallowing and inadequate nutrition.

Laryngorhinootologie $\underline{84}, 156-178$. 


\section{Moyers R:}

Handbook of orthodontics for the student and general practitioner.

3. Auflage; Medical Publishers Verlag, Chicago 1973

\section{Nilsson H, Ekberg O, Olsson R, Kjellin O, Hindfelt B (1996):}

Quantitative assessment of swallowing in healthy adults.

Dysphagia $\underline{11}, 110-116$

\section{Nilsson H, Ekberg O, Olsson R, Hinfdelt B (1998):}

Dysphagia in stroke: a prospective study of quantitative aspects of swallowing in dysphagic patients.

Dysphagia $\underline{13}, 32-38$

\section{Ono T, Hori K, Nokubi T (2004):}

Pattern of tongue pressure on hard palate during swallowing.

Dysphagia $\underline{19}, 259-264$

\section{Orchardson R, Cadden S (2009):}

Mastication and swallowing: functions, performance and mechanisms.

Dent Update $\underline{36}, 327-330,332-334,337$

Otapowicz D, Sobaniec W, Okurowska-Zawada B, Artemowicz B, Sendrowski K, Kulak W, Bockowski L, Kuzia-Smigielska J (2010):

Dysphagia in children with infantile cerebral palsy.

Ady Med Sci 55, 222-227

Ozgursoy O, Salassa J (2010):

Functional and manofluorographic outcomes after transoral endoscopic pharyngoesophageal diverticulostomy.

Arch Otolaryngol Head Neck Surg 136, 463-467

\section{Palmer JB (1989):}

Electromyography of the muscles of oropharyngeal swallowing: basic concepts.

Dysphagia $\underline{3}, 192-198$ 
Panebianco V, Ruoppolo G, Pelle G, Schettino I, Roma R, Bernardo S, De Vicentiis C, Longo L, Passariello R (2010):

Morpho-functional patterns of physiologic oropharyngeal swallowing evaluated with dynamic fast MRI.

Eur Arch Otorhinolaryngol 267, 1461-1466

Parkin D, Bray F, Ferly J, Pisani P (2001):

Estimating the world cancer burden: Globocan 2000.

Int J Cancer 94, 153-156

Pauloski BR, Rademaker AW, Logemann JA, McConnel FM, Heiser MA, Cardinale S, Lazarus CL, Pelzer H, Stein D, Beery Q (2004):

Surgical variables affecting swallowing in patients treated for oral/oropharyngeal cancer.

Head Neck 26 , 625-636

\section{Pearson G, Kirschner R (2011):}

Surgery for cleft palate and velopharyngeal dysfunction.

Semin Speech Lang 32, 179-190

Peng C, Jost-Brinkmann P, Yoshida N, Miethke R, Lin C (2003):

Differential diagnosis between infantile and mature swallowing with ultrasonography.

Eur J Orthod 25, 451-456.

Peng C, Jos-Brinkmann P, Yoshida N, Chou H, Lin C (2004):

Comparison of tongue functions between mature and tongue-thrust swallowing- an ultrasound investigation.

Am J Orthod Dentofacial Orthop 125, 562-570

Pereira N, Motta A, Vicente L (2008):

Swallowing reflex: analysis of the efficiency of different stimuli on healthy young individuals.

Pro Fono $\underline{20}, 159-164$

\section{Pomerenke WT (1928):}

A study of the sensory areas eliciting the swallowing reflex.

Am J Physiol 며, 36-41 
Prasse J, Kikano G (2008):

An overview of pediatric dysphagia.

Clin Pediatr (Phila) $\underline{48}, 247-251$

Proffit WR (1972):

Lingual pressure patterns in the transition from tongue thrust to adult swallowing.

Arch Oral Biol 17, 555-563

Proffit WR (1978):

Equilibrium Theory Revisted: Factors Influencing Position of the Teeth.

Angle Orthod $\underline{48}, 175-186$

Proffit WR, Chastain BB, Norton LA (1969):

Linguopalatal pressure in children.

Am J Orthod 55, 154-166

\section{Prosiegel M, Weber S:}

Dysphagie: Diagnostik und Therapie: Ein Wegweiser für kompetentes Handeln (Praxiswissen Logopädie).

Springer Verlag, Berlin Heidelberg 2009

\section{Prosiegel M, Heintze M, Wagner-Sonntag E, Hannig C, Wuttge-Hanning A,} Yassouridis A (2002):

Deglutition disorders in neurological patients. A prospective study of diagnosis, pattern of impairment, therapy and outcome.

Nervenarzt $\underline{73}, 364-370$

Prosiegel M, Schelling A, Warner-Sonntag E (2004):

Dysphagia and multiple sclerosis.

Int MS J $\underline{11}, 22-31$

\section{Prosiegel M, Hölling R, Heintze M, Wagner-Sonntag E, Wiseman K (2005):}

The localization of central pattern generators for swallowing in humans--a clinicalanatomical study on patients with unilateral paresis of the vagal nerve, Avellis' syndrome, Wallenberg's syndrome, posterior fossa tumours and cerebellar hemorrhage.

Acta Neurochirur Suppl 93, 85-88 


\section{Rakosi T (1970):}

Variations in deglutition.

Fortschr Kieferorthop $\underline{31}, 81-86$

\section{Redford-Badwal D, Mabry K, Frassinelli J (2003):}

Impact of cleft lip and/or palate on nutritional health and oral-motor development.

Dent Clin North Am $\underline{47}, 305-317$

\section{Reid J (2004):}

A review of feeding interventions for infants with cleft palate.

Cleft Palate Craniofac J $\underline{41}, 268-278$

\section{Reid J, Reilly S, Kilpratrick N (2007):}

Sucking performance of babies with cleft conditions.

Cleft Palate Craniofac J $\underline{44}, 312-320$

Restivo D, Marchese-Ragona R, Patti F, Solaro C, Maimone D, Zappala G, Pavone A (2011):

Botulinum toxin improves dysphagia associated with multiple sclerosis.

Eur J Neurol $\underline{18}, 486-490$

\section{Rix R (1961):}

Some problems for consideration.

Practitioner $\underline{11}$, 289-296

\section{Robbins J (1996):}

Normal swallowing and aging.

Semin Neurol 16, 309-317

Robbins J, Levine R, Wood J, Roecker EB, Luschei ES (1995):

Age effects on lingual pressure generation as a risk factor for dysphagia.

J Gerontol A Biol Sci Med Sci $\underline{50}$, M257-262 


\section{Robin DA, Goel A, Somodi LB, Luschei ES (1992):}

Tongue strength and endurance: relation to highly skilled movements.

J Speech Hear Res $\underline{35}$, 1239-1245

\section{Rohen JW, Lütjen-Drecoll E:}

Funktionelle Anatomie des Menschen: Lehrbuch der makroskopischen Anatomie nach funktionellen Gesichtspunkten.

11 Auflage; Schattauer Verlag, Stuttgart 2006

\section{Ruan W, Chen M, Gu Z, Lu Y, Su J, Guo Q (2005):}

Muscular forces exerted on the normal deciduous dentition.

Angle Orthod 75, 875-890

Sánchez-Ruiz I, González Landa G, Pérez Gonález V, Diez Rodríguez R, LópezCedrún J, Miró Viar J, García Minaur S, de Celis Vara R, Sánchez Fernández L (1999):

Integrated treatment of cleft lip and palate. Organization of a treatment team.

Cir Pediatr $\underline{12}, 4-10$

Santamato A, Panza F, Solfrizzi V, Russo A, Frisardi V, Megna M, Ranieri M, Fiore P (2009):

Acoustic analysis of swallowing sounds: a new technique for assessing dysphagia.

J Rehabil Med 1ㅜ, 639-645

\section{Schott J, Rossor M (2003):}

The grasp and other primitive reflexes.

J Neurol Neurosurg Psychiatry $\underline{74}$, 558-560

\section{Schröter-Morasch H:}

Anamnesebogen zur klinischen Erfassung von Schluckstörungen nach Hirnverletzungen.

Bergmann Publishing Verlag, Dortmund 1994 


\section{Schwestka-Polly R, Engelke W, Engelke D (1992):}

The importance of electromagnetic articulography in studying tongue motor function in the framework of an orthodontic diagnosis.

Fortschr Kieferothop 53, 3-10

\section{Selley W, Ellis R, Flack F, Brooks W (1990):}

Coordination of sucking, swallowing and breathing in the newborn: its relationship to infant feeding and normal development.

Br J Disord Commun 25, 311-327

\section{Shah C, Wong D (1980):}

Management of children with cleft lip and palate.

Can Med Assoc J 122, 19-24

\section{Shaker R, Cook IJ, Dodds WJ, Hogan WJ (1988):}

Pressure flow dynamics of the oral phase of swallowing.

Dysphagia $\underline{3}, 79-84$

\section{Shellhart W, Moawad M, Matheny J, Paterson R, Hicks E (1997):}

A prospective study of lip adaptation during six months of simulated mandibular dental arch expansion.

Angle Orthod 67, 47-54

Simental AA, Carrau RL (2004):

Assessment of swallowing function in patients with head and neck cancer.

Curr Oncol Rep $\underline{6}, 162-165$

Smithard DG, O'Neill PA, England RE, Park CL, Wyatt R, Martin DF, Morris J (1997):

The natural history of dysphagia following a stroke.

Dysphagia $\underline{12}, 188-193$

\section{Steele CM, Van Lieshout P (2004):}

Use of electromagnetic midsagittal articulography in the study of swallowing.

J Speech Lang Hear Res 47, 342-352 


\section{Steele CM, Van Lieshout P (2008):}

The dynamics of lingual-mandibular coordination during liquid swallowing.

Dysphagia $\underline{23}, 33-46$

\section{Steele CM, Van Lieshout P (2009):}

Tongue movements during water swallowing in heathy young and older adults.

J Speech Lang Hear Res 르, 1255-1267

\section{Straub W (1951):}

The etiology of the perverted swallowing habit.

Am. J. Orthod 37, 603-610

Suarez-Cunqueiro MM, Schramm A, Schoen R, Soane-Lestón J, Otero-Cepeda XL, Bormann KH, Kokemueller H, Metzger M, Diz-Dios P, Gellrich NC (2008):

Speech and swallowing impairment after treatment of oral and oropharyngeal cancer.

Arch Otolaryngol Head Neck Surg 134, 1299-1304

Suntrup S, Warnecke T, Kemmling A, Teismann IK, Hamacher C, Oelenberg S, Dziewas R (2012):

Dysphagia in patients with acute striatocapsular hemorrhage.

J Neurol 259, 93-99

Takatsuji H, Zakir H, Mostafeezur R, Saito I, Yamada, Y., Yamamura, K, Kitagawa J (2012):

Induction of the swallowing reflex by electrical stimulation of the posterior oropharyngeal region in awake humans.

(Dysphagia im Druck)

\section{Takemoto H (2001):}

Morphological analyses of the human tongue musculature for three-dimensional modeling.

J Speech Lang Hear Res $\underline{44}$, 95-107

\section{Tamine K, Ono T, Hori K, Kondoh J, Hamanaka S, Maeda Y (2010):}

Age related changes in tongue pressure during swallowing.

J Dent Res $\underline{89}$, 1097-1101 


\section{Taniguchi H, Tsukada T, Ootaki S, Yamada Y, Inoue M (2008):}

Correspondence between food consistency and suprahyoid muscle activity, tongue pressure, and bolus transit times during the oropharyngeal phase of swallowing.

J Appl Physiol 105, 791-799

\section{Thexton A, Crompton A, Owerkowicz T, German R (2004):}

Correlation between intraoral pressures and tongue movements in the suckling pig.

Arch Oral Biol $\underline{49}, 567-575$

\section{Thüer U, Janson T, Ingervall B (1985):}

Application in children of a new method for the measurement of forces from the lips on the teeth.

Eur J Orthod , 63-78

\section{Thüer U, Sieber R, Ingervall B (1999):}

Cheek and tongue pressures in the molar areas and the atmospheric pressure in the palatal vault in young adults.

Eur J Orthod 21, 299-309

\section{Tomes C (1873):}

The bearing of the development of the jaws on irregularties.

Dental Cosmos 15, 292

\section{Tuchman D (1988):}

Dysfunctional swallowing in the pediatric patient: clinical considerations.

Dysphagia 2, 203-208

\section{Utanohara Y, Hayashi R, Yoshikawa M, Yoshida M, Tsuga K, Akagawa Y (2008):}

Standard values of maximum tongue pressure taken using newly developed disposable tongue pressure measurement device.

Dysphagia $\underline{23}, 286-290$

\section{Vaiman M, Eviatar E (2009):}

Surface electromyography as a screening method for evaluation of dysphagia and odynophagia.

Head Face Med 20, 5-9 
van der Maarel-Wierink C, Vanobbergen J, Bronkhorst E, Schols J, de Baat C (2011):

Risk factors for aspiration pneumonia in frail older people: a systematic literature review.

J Am Med Dir Assoc 12, 344-354

\section{Vitorino J (2010):}

Effect of age on tongue strength and endurance scores of healthy Portugese speakers.

Int J Speech Lang Pathol 12, 237-243

\section{Wein B, Angerstein W, Klajman S, Zimmermann R, Skopnik H (1993):}

Tongue ultrasound pressure measurement in infantile sucking. Initial results.

Klin Padiatr 205, 103-106

\section{Weinstein S, Haack D, Morris L, Synder B, Attaway H (1963):}

On an equilibrium theroy of tooth position.

Angle Ortho $\underline{33}, 1-26$

\section{Wendler J, Seidner W, Eysholdt U:}

Lehrbuch der Phoniatrie und Pädaudiologie.

4. Auflage; Thieme Verlag, Stuttgart 2005

\section{Winders R (1956):}

A study in the development of an electronic technique to measure the forces exerted on the dentition by the perioral and lingual musculature.

Am J Orthod $\underline{42}, 645-657$

\section{Winders R (1958):}

Forces Exerted on the Dentition by the Perioral and Lingual Musculature During Swallowing.

Angle Orthod 28, 226-235

\section{Window AL:}

Strain gauge technology.

2. Auflage; Springer Verlag, Niederlande 1992 


\section{Wu CH, Hsiao TY, Ko JY, Hsu MM (2000):}

Dysphagia after radiotherapy: endoscopic examination of swallowing in patients with nasopharyngeal carcinoma.

Ann Otol Rhinol Laryngol 109, 320-325

\section{Wynder EL, Bross IJ, Feldman RM (1957):}

A study of the etiological factors in cancer of the mouth.

Cancer $\underline{10}, 1300-1323$

\section{Youmans S, Stierwalt J (2006):}

Measures of tongue function related to normal swallowing.

Dysphagia 21, 102-111

\section{Youmans S, Youmans G, Stierwalt J (2009):}

Differences in tongue strength across age and gender: is there a diminished strength reserve?

Dysphagia 24, 57-65

\section{Zirkler HU:}

Polysensographische Untersuchung der orofazialen Basisfunktion im Wachzustand und während der Nachtruhe.

Med. Diss. Göttingen 2002 


\section{Danksagung}

$\mathrm{Zu}$ besonderem Dank bin ich Herrn Prof. Dr. Dr. Wilfried Engelke verpflichtet. Er hat mir nicht nur das Thema der vorliegenden Arbeit gegeben, sondern auch die Freude und Interesse an der wissenschaftlichen Arbeit gezeigt.

Weiterhin möchte ich Frau Priv.-Doz. Dr. Christiane Völter danken für ihre kontinuierliche Motivation und großzügige Unterstützung bei der Datenerhebung.

Herrn Simon Schneider, Frau Dr. Imme Haubitz und Herrn David Ellenberger gebührt mein Dank für die Hilfe bei der statistischen Analyse. 


\section{Lebenslauf}

Am 23. August 1984 wurde ich, Petra Santander Martinez, in Mechernich als Tochter der Krankenschwester Petra Santander Martinez und des Arztes Dr. Hernán Santander Martinez geboren.

Von 1991 bis 2002 besuchte ich die Deutsche Schule in Villarrica, Chile. Im Dezember 2002 legte ich das chilenische Abitur ab.

In März 2004 nahm ich das Studium der Zahnheilkunde an der Universidad de La Frontera in Temuco, Chile auf. Die Zahnärztliche Prüfung absolvierte ich am 9. Dezember 2009 erfolgreich. Nach dem Abschluss meines Studiums in Chile absolvierte ich einen 15 - monatigen stipendiumgefördeten Qualifizierungsaufenthalt in der Universitätsmedizin Göttingen. Seit dem 15. Februar 2012 bin ich als Assistenzärztin in der Abteilung für Mund-, Kiefer- und Gesichtschirurgie der Universitätsmedizin Göttingen tätig. 